\title{
Ion-beam mixing of Fe/Si bilayers
}

\author{
Dissertation \\ zur Erlangung des Doktorgrades \\ der Mathematisch-Naturwissenschaftlichen Fakultäten \\ der Georg-August-Universität zu Göttingen
}

vorgelegt von

Velimir Milinović

aus Belgrad, Serbia \& Montenegro

Göttingen 2005 
D7

Referent: Prof. Dr. Dr. h.c. Klaus Peter Lieb

Korreferent: Prof. Dr. Angela Ritzy

Tag der mündlichen Prüfung: 27.10.2005 
1. Summary _ 2

2. Introduction

2.1. Interaction of ions with solids ___ 3

2.1.1.Energy loss: __ 3

2.1.2. Collision cascades __ 6

2.1.3. Ion-beam induced mixing___ 6

2.2. The Fe-Si system __ 9

2.3. Previous results of ion beam mixing of Fe/Si bilayers ___ 11

2.4. The aim of this work 12

3. Experimental techniques ___ 13

3.1. Film preparation _ـ 13

3.2. Rutherford Backscattering Spectroscopy (RBS)

3.3. Mössbauer spectroscopy _ـ 18

3.4. The Magneto-Optical Kerr Effect (MOKE) __ 28

3.5. X-Ray Diffraction (XRD) _ 31

4. Nitrogen irradiation and thermal annealing __ 33

4.1. Sample preparation and ${ }^{14} \mathrm{~N}^{++}$ion irradiation $\_34$

4.2. Results and discussion _ـ 34

4.2.1. Phase formation _ـ 34

4.2.2. Ion beam mixing _ 38

4.3. Annealing: results and discussion__ 40

5. Effects of substrate amorphization and ion charge

5.1. Sample preparation _ 45

5.2. Results and discussion __ 46

6. Swift ion irradiation with $350 \mathrm{MeV} \mathrm{Au}$ ions

6.1. Sample preparation

6.2. Results and discussion _ـ 55

7. References __ 64

Acknowledgements__ 68 


\section{Summary}

The present study focuses on the changes in the structural and magnetic properties in Fe/Si bilayers induced by heavy-ion irradiation $\left({ }^{14} \mathrm{~N},{ }^{40} \mathrm{Ar},{ }^{84} \mathrm{Kr},{ }^{132} \mathrm{Xe},{ }^{197} \mathrm{Au}\right)$. Polycrystalline Fe layers of 30-70 nm thickness were prepared by electron-gun evaporation, pulsed laser deposition or ion-beam-assisted deposition. Rutherford backscattering and Mössbauer spectroscopy, X-ray diffraction and magneto-optical Kerr effect were used as analysing techniques, as described in Chapter 3. With respect to previous work, in which mainly noble-gas ions of low charge state and several hundred keV energy were investigated, several "non-standard" implantation conditions were considered:

(1) The use of chemically active ions such as nitrogen demonstrated the competition between nitride formation and ion beam mixing at the interface (Chapter 4). During implantation only iron nitrides were formed; even at the highest nitrogen fluence no silicides were formed. After heating the irradiated samples in vacuum up to $700{ }^{\circ} \mathrm{C}$, the iron-rich phases $\varepsilon-\mathrm{Fe}_{3} \mathrm{~N}$ and $\gamma^{\prime}-\mathrm{Fe}_{4} \mathrm{~N}$ were formed. Starting at $600{ }^{\circ} \mathrm{C} \beta$ $\mathrm{FeSi}_{2}$ was identified [1-1, 2].

(2) For several noble-gas ions, the effects of ion charge (e.g. $250 \mathrm{keV} \mathrm{Xe}$ versus $\mathrm{Xe}^{17+}, 180 \mathrm{keV} \mathrm{Kr}^{+}$versus $\mathrm{Kr}^{11+}, 100 \mathrm{keV} \mathrm{Ar}^{+}$versus $\mathrm{Ar}^{8+}$ ) and low-energy

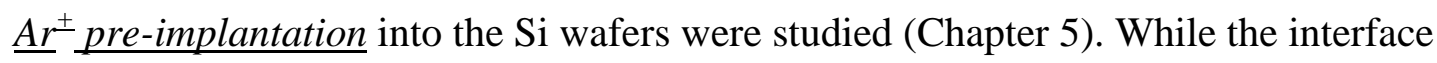
mixing rate does not depend on the ion charge, pre-amorphization of the Si wafer enhances the mixing rate by a factor 2 .

(3) Finally, experiments were conducted for swift $350 \mathrm{MeV}{ }^{197} \underline{\mathrm{Au}}^{26+}$ ions to measure the mixing rate and the magnetic anisotropy in the regime of electronic stopping (Chapter 6). A very high mixing rate of $\Delta \sigma^{2} / \Phi=55$ (5) $\mathrm{nm}^{4}$ was found and attributed to thermal spikes due to the very high energy density of about $40 \mathrm{keV} / \mathrm{nm}$ deposited in the Fe/Si couple [1-3, 4]. 


\section{Introduction}

Investigations in the field of radiation damage started sixty years ago with the advent of nuclear energy. Most of the studies in this early period were devoted to the modification of metallic compounds under neutron irradiation. Later on, the use of implantation for doping semiconductors [2-1] and producing hard coatings expanded strongly [2-2]. Finally, the formation of new phases by ion irradiation, and atomic mixing of thin films moved into focus, and it is still an active research area. Ion irradiation carried out on almost all classes of materials, from metals to living cells. As radiation induces non-equilibrium states of matter, new materials with novel properties can be created. Many applications of ion irradiation have been developed for the structuring of micro- and nano-materials of high technological interest.

\subsection{Interaction of ions with solids}

\subsubsection{Energy loss:}

When an energetic ion penetrates solid matter, it loses its kinetic energy mainly via two processes: either by elastic collision with the nuclei of the target (nuclear stopping); or inelastically by electronic excitation and ionization (electronic stopping) $[2-3,4]$ :

$$
\frac{d E}{d x}=\left(\frac{d E}{d x}\right)_{n}+\left(\frac{d E}{d x}\right)_{e}
$$

The energy loss during interaction of the ion and the target atoms is given by:

$$
\left(\frac{d E}{d x}\right)_{n}=\sigma \cdot \frac{m+M}{4 \pi e^{2} Z_{m} Z m}
$$

where $m, M, Z_{m}$ and $Z$ are the masses and atomic numbers of the ion and target atom, respectively, and $\sigma$ is the cross section for transferring the energy $T$ from the ion to the target atom. The transferred energy $T$ for nuclear stopping is:

$$
T=\frac{4 m M \sin ^{2}\left(\frac{\phi}{2}\right)}{(m+M)^{2}} E_{i}
$$

where $\phi$ is the scattering angle and $E_{i}$ is the initial energy of the ion. 
The electronic stopping can be divided in to two regions separated by the velocity $v_{o} \cdot \mathrm{Z}_{\mathrm{i}}^{2 / 3}$, where $v_{o}$ is the Bohr velocity. When the ion velocity $v_{i}$ is in the range of $0.1 \cdot v_{o}$ to $v_{o} \cdot \mathrm{Z}_{\mathrm{i}}^{2 / 3}$, the electronic energy loss is approximately proportional to $v_{i}$ (or $\mathrm{E}_{\mathrm{i}}^{1 / 2}$ ); while for $v_{i}>v_{o} \cdot \mathrm{Z}_{\mathrm{i}}^{2 / 3}$ the electronic energy loss is proportional to $1 / v_{\mathrm{i}}^{2}$ (or $1 / E_{i}$ ). Nuclear and electronic energy losses are schematically shown in Fig 2.1.

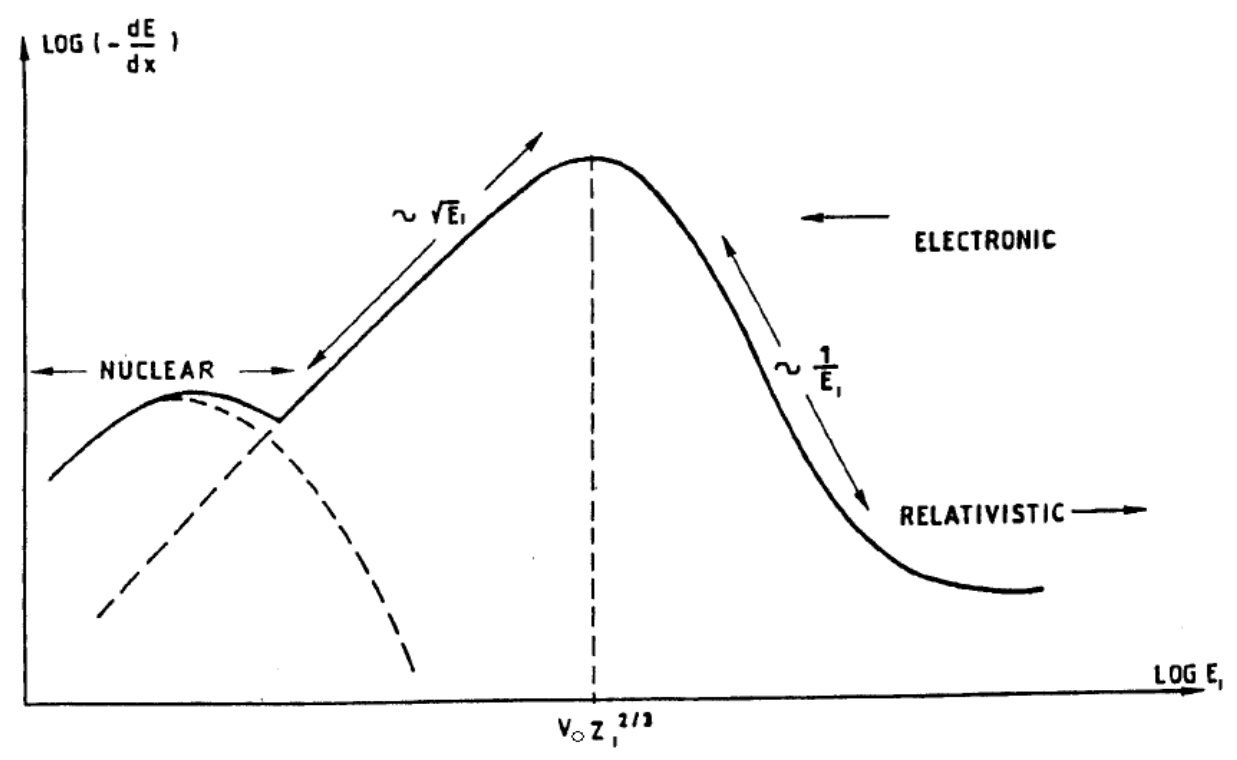

Figure 2.1 Energy loss $\mathrm{dE} / \mathrm{dx}$ of the ion, with energy $\mathrm{E}_{\mathrm{i}}$ and atomic number $\mathrm{Z}_{\mathrm{i}}$, during penetration of a solid.

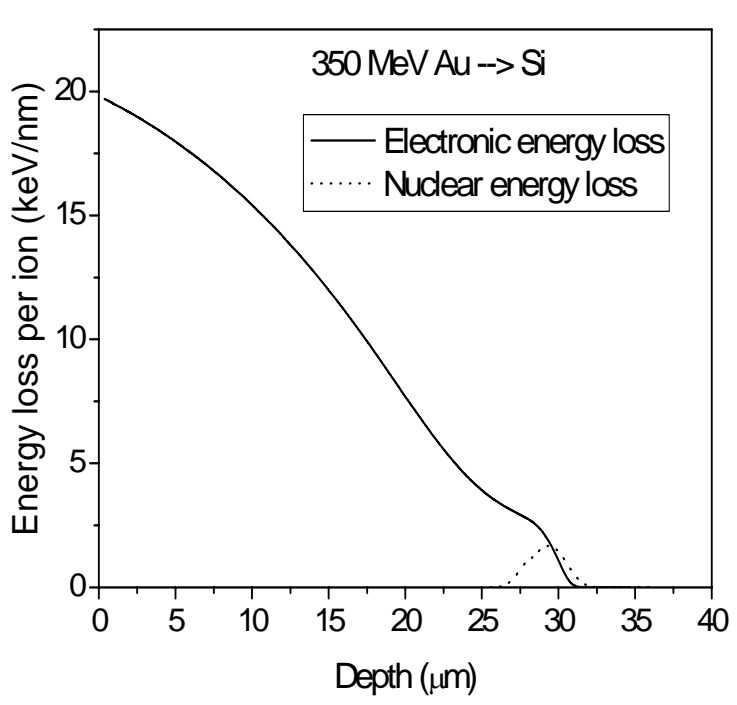

(a)

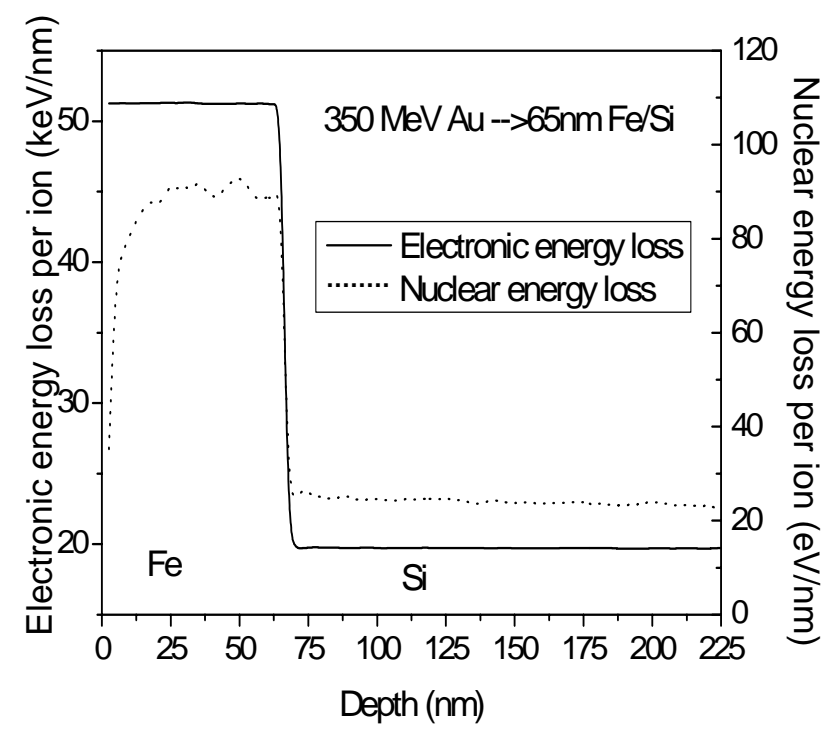

(b)

Figure 2.2 Energy loss of $350 \mathrm{MeV}$ Au ion in Si (a) and 65nm Fe/Si (b). 
Fig 2.2 shows the results of the Monte Carlo simulation [2-5] for nuclear and electronic losses for $350 \mathrm{MeV}$ gold ions in silicon and in 65nm Fe/Si bilayer. For this ion energy in the first few micrometers electronic energy loss is dominant and about three orders of magnitude larger than for nuclear stopping.

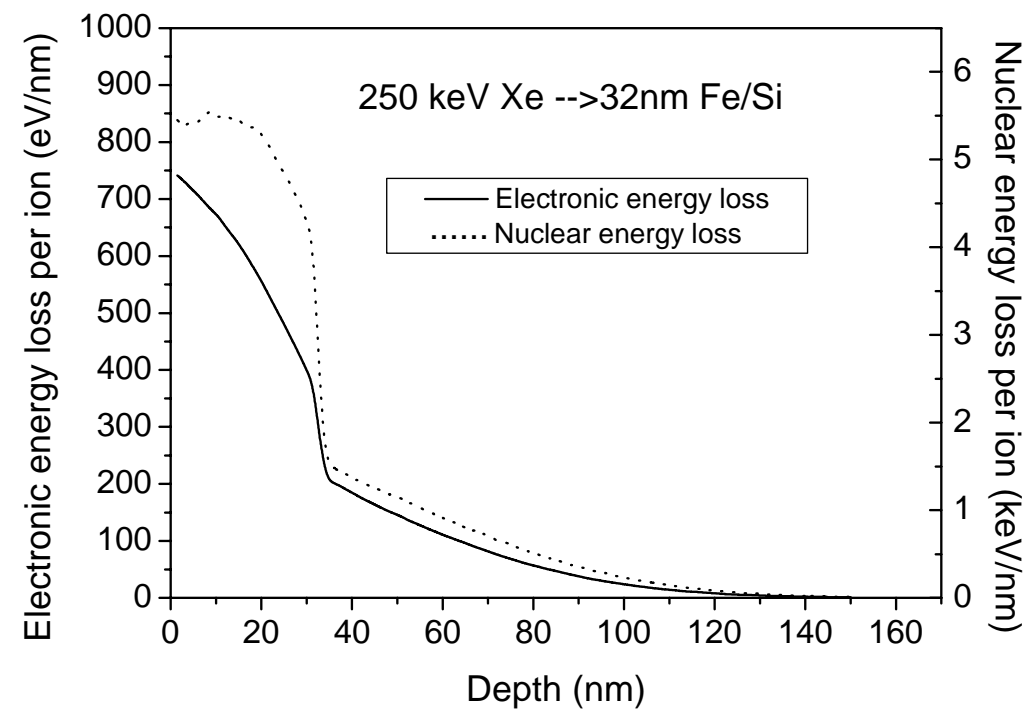

Figure 2.3 Results of the Monte Carlo simulation for nuclear and electronic loss of $250 \mathrm{keV}$ Xe ions in a 32nmFe/Si bilayer.

Both energy losses remain constant over the first few hundreds of nm. At the end of the ion path, some $30 \mu \mathrm{m}$ from the surface of the system, nuclear stopping becomes dominant. Fig 2.3. shows the situation, where nuclear loss is dominant in thin film/substrate interface region. In this figure, results of the Monte Carlo simulation for nuclear and electronic loss of $250 \mathrm{keV}$ Xe ions in a 32nmFe/Si bilayer are shown. In the case of ion beam mixing experiments with $350 \mathrm{MeV}$ gold ion beam, the effect of the electronic energy loss is crucial, while the thickness of the thin Fe top layer is negligible; variations of few tens of nm will not cause any change in energetic losses (Fig 2.2). On the other hand, in the case of ion beam experiments with ions in the 100 - $400 \mathrm{keV}$ energy range, nuclear energy loss is dominant, and a thickness variation of the thin layer should be carefully controlled. 


\subsubsection{Collision cascades}

Molecular dynamic simulations suggest three phases during the interaction of the energetic ion and solid: collision cascade phase, “thermal spike” phase, and relaxation phase. The collision cascade phase lasts for a few tenths of a picosecond. The primary collision between the ion and a target atom induces a series of secondary, ternary, etc., recoils, as long as the energy transfer exceeds the displacement threshold $E_{d}$. This displacement threshold is defined as the minimum energy required to produce a stable interstitial-vacancy (Frenkel) pair. Typical values of $E_{d}$ are 20-30 eV. The thermal spike phase presents a specific condition of the collision cascade when all atoms in the volume of the cascade are in thermal motion. This leads to local melting, and the distribution of the atoms can be described by the Maxwell-Boltzman distribution assuming that spikes can only exist in a space-filling cascade. The upper threshold energy of the target atoms can be found [2-6]:

$$
E_{c}=0.039 \cdot Z^{2.23}
$$

Bearing in mind that the lower threshold energy for starting a cascade is $E_{d}$, the energy range for thermal spike formation is: $E_{d}<E_{\text {projectile }}<E_{c}$. In general, for a material with an average atomic number of less than 20 , no thermal spikes are expected. The duration of a thermal spike is approximately $10^{-11} \mathrm{~s}$.

The "relaxation phase" is the last phase in the collision process and represents the relaxation of the non-equilibrium regions left after the thermal spike phase.

\subsubsection{Ion-beam induced mixing}

Ion-beam mixing (IBM) techniques use energetic ion beams for atomic mixing of the solid materials A and B, either as bilayers or multilayers. Let us consider the case of the thin film A deposited onto a substrate B and irradiated with a heavy ion beam, assuming that the ion range is close to the thickness of film A. With properly chosen irradiation parameters a broadening of the concentration profiles $\left(\Delta \sigma^{2}\right)$ in the interface region of materials A and B may occur. The broadening of the concentration profile $\left(\Delta \sigma^{2}\right)$ is defined as:

$$
\Delta \sigma^{2}=\Delta \sigma_{i r r}^{2}-\Delta \sigma_{a d}^{2}(2.5)
$$

where $\Delta \sigma^{2}$ ad and $\Delta \sigma^{2}$ irr are squares of the half the distance between $16 \%$ and $84 \%$ of the maximum concentration of a certain element in the A/B interface region (see Fig 
2.4) for the as-deposited and the irradiated system respectively. In most cases the broadening of the concentration profiles is linear to the applied ion fluence:

$$
\Delta \sigma^{2}=k \cdot \Phi \quad(2.6)
$$

where $k$ is the mixing rate.

A similar linear relation of the concentration profiles is obtained during thermal annealing of the A/B system is shown in Eq 2.7:

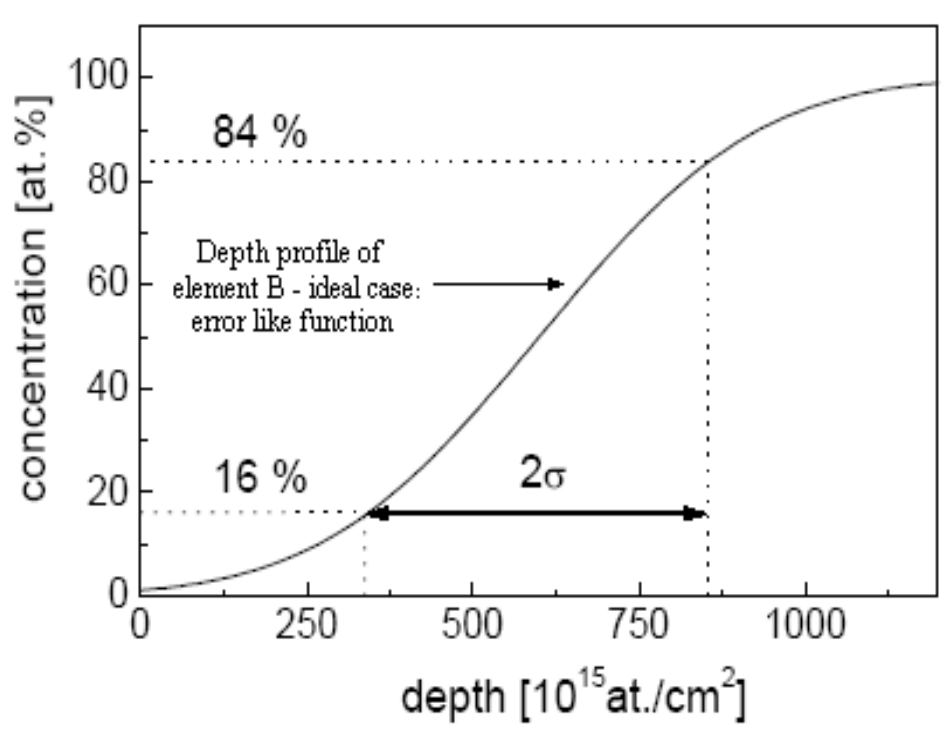

Figure 2.4 Determination of the variation of the concentration.

$$
\Delta \sigma^{2}=2 \cdot D \cdot t
$$

where $D$ is diffusivity and $\mathrm{t}$ is the diffusion time. The diffusivity $D$ is given by the relation:

$$
D=v \cdot \lambda^{2} / 6
$$

where $v$ is the average velocity and $\lambda^{2}$ is the mean square distance of the thermally activated atomic jumps. By making the correlation between Eq 2.6

and Eq 2.8 and replacing $v$ with the number of atoms replaced per fluence unit $n_{r} / N$, where $n_{r}$ is the number of relocated atoms per incoming ion and path length and $N$ is the atomic density of the target, the following relation for the mixing rate $k$ is obtained:

$$
k=\Delta \sigma^{2} / \Delta \Phi=n_{r} / 3 \cdot N \cdot \lambda_{r}^{2}
$$

The task of all theoretical models is to find suitable expressions for the quantities $n_{r}$ and $\lambda_{r}$ which successfully describe experimental results. The basic models, dealing with the IBM process, are: the ballistic model, the global spike model and the local spike model.

\section{a) Ballistic mixing}

This model only assumes that independent binary collisions between target atoms contribute to the mixing rate $k$, i.e. during the ballistic cascade phase. The final relation for ballistic mixing has the following form [2-7, 8]: 


$$
k_{\text {ball }}=\frac{1}{3} \Gamma_{0} \xi \frac{F_{D} R_{d}^{2}}{N E_{d}}
$$

where $\xi$ is a kinematic factor, $\xi=\left[4 \mathrm{mM} /(\mathrm{mM})^{2}\right]^{1 / 2}, \Gamma_{0}=0.608$ is a dimensionless constant, $N$ is the atomic density of the target, $R_{d}$ is the minimum separation distance for the production of a stable Frenkel pair $(\approx 1 \mathrm{~nm})$. $F_{D}$ is the deposited energy per ion per unit length and defined by:

$$
F_{D}(x)=\left(2 n_{v}(x)+n_{r}(x)\right) \cdot E_{D}
$$

where $n_{v}(x)$ is the number of vacancies produced per nm and $n_{r}(x)$ is the number of replacement collisions. The $F_{D}$ values are obtained by the SRIM code.

b) Global spike mixing

The model for the global spike mixing is based on the assumption of a cylindrical thermal spike around the ion path, and has the following form [2-6, 9]:

$$
k_{g b}=k_{1} N^{-5 / 3}\left(\frac{F_{D}}{\Delta H_{c o h}}\right)^{2}\left(1+\frac{k_{2} \Delta H_{r}}{\Delta H_{c o h}}\right)
$$

where $k_{1}=0.35 \mathrm{~nm}$ and $k_{2}=27.4$ are phenomenological constants, $\Delta H_{r}$ is the reaction enthalpy and $\Delta H_{c o h}$ is the cohesive energy of the reaction products.

c) Local spike mixing

The local spike model assumes the formation of local spherical spikes along the collision cascades [2-10]:

$$
k_{l s}=k_{1}^{\prime} \frac{Z_{t}^{1.5}}{N^{4 / 3} \Delta H_{c o h}^{5 / 3}}\left(1+\frac{5}{6} k_{2}^{\prime} \frac{\Delta H_{r}}{\Delta H_{c o h}}\right) F_{D}
$$

where the phenomenological constants $k_{1}$ ' and $k_{2}$ ' are independent of the target material.

It was found that the ballistic effects are often very small and that local or global thermal spikes dominate atomic mixing in metal/silicon bilayers irradiated with heavy ions. 


\subsection{The Fe-Si system}

Pure iron exists in three allotropic phases: $\alpha, \gamma$, and $\delta . \alpha$-Fe forms a bcc crystal structure and has an atomic weight of 55.847. The transformation point between $\alpha$-Fe and $\gamma$-Fe is $911^{\circ} \mathrm{C}$. $\gamma$-Fe has an fcc crystal structure and an atomic weight of 55.847. The transformation point at which bcc $\delta$-Fe is formed is $1392{ }^{\circ} \mathrm{C}$. The melting point of iron is at $1536{ }^{\circ} \mathrm{C}$. At $20^{\circ} \mathrm{C}$, the density of $\alpha, \gamma$, and $\delta$-Fe is $7.866,8.33$, and $7.86 \mathrm{~g} / \mathrm{cm}^{3}$ respectively.

Silicon has a diamond crystal structure. Its melting point is at $1412{ }^{\circ} \mathrm{C}$, its atomic weight is 28.086 and its density $2.33 \mathrm{~g} / \mathrm{cm}^{3}$.

The Fe-Si binary phase diagram is rather complicated, and consists of numerous compounds and solid solutions in different allotropic phases, which may coexist (see Fig. 2.5).

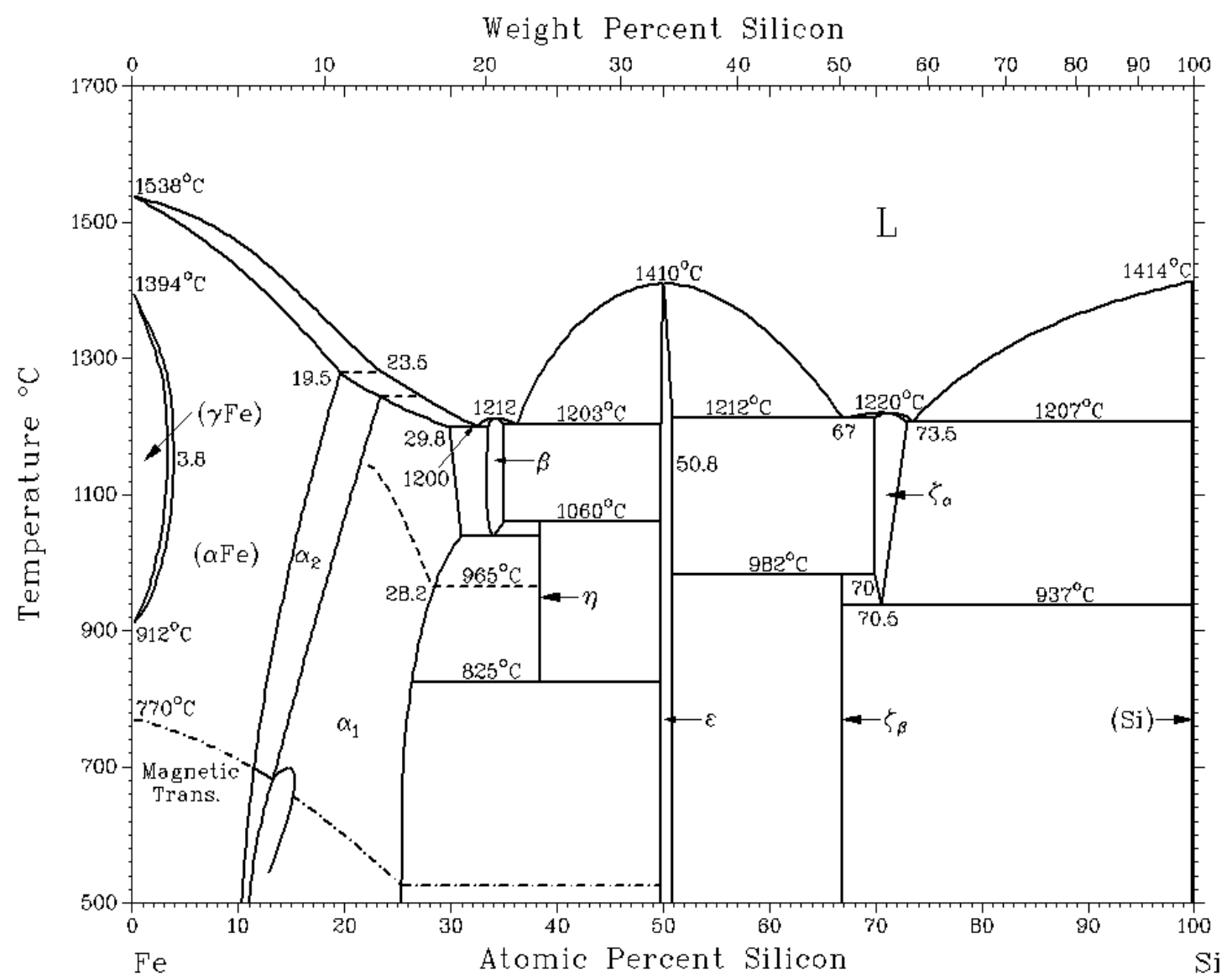

Figure 2.5 Fe-Si binary phase diagram. 
$\mathrm{Fe}_{3} \mathrm{Si}$ and $\mathrm{FeSi}$ are low-temperature phases. Iron-disilicide exists in three different phases: in a metastable cubic $\gamma$-FeSi 2 phase, a stable orthorhombic $\beta-\mathrm{FeSi}_{2}$ phase, and a stable tetragonal $\alpha-\mathrm{FeSi}_{2}$ phase. FeSi (bulk unstable) and $\gamma-\mathrm{FeSi}_{2}$ are metallic silicides. The $\beta-\mathrm{FeSi}_{2}$ and $\varepsilon$-FeSi phases are semiconductors, and $\mathrm{Fe}_{3} \mathrm{Si}$ and bulk unstable $\gamma-\mathrm{FeSi}_{2}$ are magnetic compounds. The Mössbauer parameters are well documented for this system [2-11, 12, 13], which makes this spectroscopy ideal for the investigation of mixing effects. 


\subsection{Previous results of ion beam mixing of Fe/Si bilayers}

Ion beam mixing and silicide formation in metal/silicon bilayers have been extensively studied over many years, and the Fe/Si system is no exception [2-14, 15, $16,17,18,19]$. All these experiments were performed with chemically non-reactive ion beams (Ar, Kr, Xe and $\mathrm{Au}$ ), at energies ranging from 80 to $700 \mathrm{keV}$, and ion fluences between $1 \times 10^{15}$ to $2 \times 10^{16}$ ions $/ \mathrm{cm}^{2}$ in a wide temperature range (from liquid nitrogen temperature to $870 \mathrm{~K}$ ). The results of the previous IBM experiments on the Fe/Si system are summarized in Table 2.1.

Table 2.1 Ion beam mixing results achieved by the previous experiments performed on the $\mathrm{Ta} / \mathrm{Si}$ and $\mathrm{Fe} / \mathrm{Si}$ bilayer systems.

\begin{tabular}{|c|c|c|c|c|c|c|}
\hline \multirow{2}{*}{ Bilayer system } & \multirow{2}{*}{ Ion } & \multicolumn{4}{|c|}{ Mixing Rate $\Delta \sigma^{2} / \Phi\left(\mathrm{nm}^{4}\right)$} & \multirow{2}{*}{ Ref } \\
\hline & & Experimental & Ballistic & Local & Global & \\
\hline $42 \mathrm{~nm} \mathrm{Fe} / \mathrm{Si}$ & $100 \mathrm{keV} \mathrm{Ar}^{+}$ & $1.3(2)$ & 0.16 & 1.3 & 0.4 & {$[2-18]$} \\
\hline $31 \mathrm{~nm} \mathrm{Fe} / \mathrm{Si}$ & $100 \mathrm{keV} \mathrm{Ar}^{+}$ & $1.5(2)$ & \multirow{2}{*}{0.18} & \multirow{2}{*}{1.4} & \multirow{2}{*}{0.5} & {$[2-18]$} \\
\hline $33 \mathrm{~nm} \mathrm{Fe} / \mathrm{Si}$ & $100 \mathrm{keV} \mathrm{Ar}^{8+}$ & $2.1(2)$ & & & & {$[2-18]$} \\
\hline $42 \mathrm{~nm} \mathrm{Fe} / \mathrm{Si}$ & $250 \mathrm{keV} \mathrm{Xe}^{+}$ & $4.8(5)$ & 0.5 & 4 & 3.5 & {$[2-17]$} \\
\hline $90 \mathrm{~nm} \mathrm{Fe} / \mathrm{Si}$ & $700 \mathrm{keV} \mathrm{Xe}^{2+}$ & $6.7(1.0)$ & 0.6 & 4.8 & 4.1 & [2-17] \\
\hline $36 \mathrm{~nm} \mathrm{Fe} / \mathrm{Si}$ & $400 \mathrm{keV} \mathrm{Au}^{+}$ & $8.5(1.5)$ & 0.9 & 7.4 & 10 & {$[2-17]$} \\
\hline
\end{tabular}

In all the cases, a linear increase in the interface variance $\Delta \sigma^{2}$ with the ion fluence $\Phi$ was obtained and the deduced mixing rates $k=\Delta \sigma^{2} / \Phi$ were well fitted with either the local or global spike model. One of the experiments was performed on 30-40 nm thick Fe layers with $\mathrm{Ar}^{+}$and $\mathrm{Ar}^{8+}$ in order to check a possible influence of the ion charge state on the mixing rate, but no clear conclusion could be drawn [2-18]. Besides the Fe - Si solid solutions and the $\varepsilon-F e S i$ phase, in some cases also the $\beta$ $\mathrm{FeSi}_{2}$ phase was produced. Since this phase has interesting features (a band gap that matches the wavelength of silica optical fibers, thus making it a promising industrial material), a special effort was made to produce single-phase $\beta-\mathrm{FeSi}_{2}$. After a finetuning of the experimental conditions, this aim was finally achieved [2-20]. 


\subsection{The aim of this work}

As pointed out before, most previous irradiation experiments in the $\mathrm{Fe} / \mathrm{Si}$ system were performed with chemically inactive ions, and at ion energies of hundreds of $\mathrm{keV}$, i.e. in the domain where nuclear stopping is dominant. In these experiments, the implanted ions served to induce or enhance atomic interdiffusion and/or phase formation via primary collisions cascades and subsequent thermal spikes. The ions were in a low charge state, and their projected range was close to the film/substrate interface.

Based on previous results, the present work aims at investigating basically novel aspects of the ion beam irradiation of Fe/Si bilayers and/or trilayers.

(a) Chapter 4 deals with the investigation on the influence of the irradiation of $\mathrm{Fe} / \mathrm{Si}$ bilayers with chemically active ions, namely nitrogen, on these bilayers. We were also interested in the possibility of the formation of iron-nitride and/or silicon nitride phases during irradiation as well as during post-annealing treatments.

(b) As mentioned before, the influence of the charge state on the mixing rate of $\mathrm{Fe} / \mathrm{Si}$ bilayers is not clear yet. In order to clarify this question, a careful comparison of the mixing rates obtained with highly and singly charged ions was performed for various ions ( $\mathrm{Ar}, \mathrm{Kr}$, and $\mathrm{Xe}$ ). In addition, in the same sets of experiments, the influence of the silicon surface pretreatment prior to the deposition of the iron thin film was studied (Chapter 5).

(c) The final part of this work (Chapter 6) is dedicated to studying the influence of the irradiation of the ${ }^{\text {nat }} \mathrm{Fe} /{ }^{57} \mathrm{Fe} / \mathrm{Si}$ trilayers with swift heavy ions, namely $350 \mathrm{MeV} \mathrm{Au}{ }^{17+}$. In contrast to all previous experiments, here nuclear stopping is negligible, and electronic stopping plays the major role. 


\section{Experimental techniques}

\subsection{Film preparation}

All the samples were made of thin solid iron films deposited on silicon wafers. The film thickness was up to $70 \mathrm{~nm}$. The films were prepared by physical vapor deposition techniques: either electron gun deposition or pulsed laser deposition (PLD). Two sets of the films, with natural iron, were prepared by either electron gun deposition in Göttingen in an ultra-high-vacuum (UHV) chamber [3-1] or by Ion Beam Assisted Deposition (IBAD) chamber at the Vinča Institute, Belgrade [3-2]. Another set of samples, containing layers of ${ }^{57} \mathrm{Fe}, 95 \%$ isotopically enriched, were grown by pulsed laser deposition on Si (100) wafers at the Institute of Materials Physics, Göttingen University [3-3]. The last set of samples, with a $20 \mathrm{~nm}$ thick layer of ${ }^{57} \mathrm{Fe}$ between the top $45 \mathrm{~nm}$ of natural iron layer and the silicon substrate, were prepared by PLD at the Leibniz Institut für Festkörper und Werkstoffforschung (IFW), Dresden.

Before mounting the Si substrates in the deposition chambers, a standard cleaning procedure was performed: moisture was removed by ultrasonic cleaning, and after that procedure, the native oxide layers on the Si-wafer surface were removed by diluted HF and deionized water. All these precautions did not provide a contaminantfree surface of the Si wafer. At atmospheric pressure, monolayers of silicon oxide are formed almost immediately (within less than a second). Even when the wafer is mounted inside the deposition chamber, which is evacuated to the typical pressure of $10^{-8}-10^{-9}$ mbar, monolayers are formed within 5-30 minutes. Consequently, all samples had some degree of surface and interface contamination, mainly by $\mathrm{H}_{2} \mathrm{O}$ vapor and/or $\mathrm{CO}_{2}, \mathrm{H}_{2}$. In order to achieve a clean surface, the Si wafers of the samples prepared in Belgrade were sputter-cleaned with a $1 \mathrm{keV} \mathrm{Ar}^{+}$ion beam prior to the deposition, but on the other hand, the pressure in the IBAD chamber was only $10^{-7}$ mbar, i.e. one order of magnitude lower than in the other cases.

\subsection{Rutherford Backscattering Spectroscopy (RBS)}

Since the first experiment with energetic alpha particles scattered from a thin gold foil was performed by Rutherford and Geiger (Nobel Prize in chemistry in 1908), the RBS technique has been under constant development and improvement and is nowadays a standard technique for analyzing depth profiles in thin films. RBS deals with the 
measurement of the energies of ions backscattered from the sample. The sample is not damaged structurally during the analysis and therefore RBS is considered nondestructive. Typically, helium ions with energies of up to $2 \mathrm{MeV}$ are used. From the energy of the backscattered particles the target's elemental composition can be deduced; from their intensity the target's elemental concentration can be resolved and from the energy loss of the particles in the specimen before and after the scattering, one determines the elemental depth distribution profile. The wide use of the RBS technique is due to its high sensitivity (mainly for heavy components, typically 0.1 at.\% in a light matrix) and depth resolution in the nm range.

\section{$\underline{\text { Kinematic factor }}$}

An incident beam of particles with mass $m$ and energy $E_{0}$ is backscattered at the surface of a sample by nuclei with mass $M_{i}$. The energy of the backscattered particles only depends on $M_{i}$ and three known fixed quantities: $E_{0}, m$, and the scattering angle $\theta$, in the laboratory frame. The kinematic factor is the ratio between the energy of the backscattered and incident particles and it is given by:

$$
K_{\mathrm{i}} \stackrel{\text { def }}{=} \frac{\mathrm{E}_{\mathrm{i}}}{\mathrm{E}_{0}}=\left(\frac{\left(\mathrm{M}_{\mathrm{i}}^{2}-\mathrm{m}^{2} \cdot \sin ^{2} \theta\right)^{1 / 2}+\mathrm{m} \cdot \cos \theta}{\mathrm{M}_{\mathrm{i}}+\mathrm{m}}\right)^{2}
$$

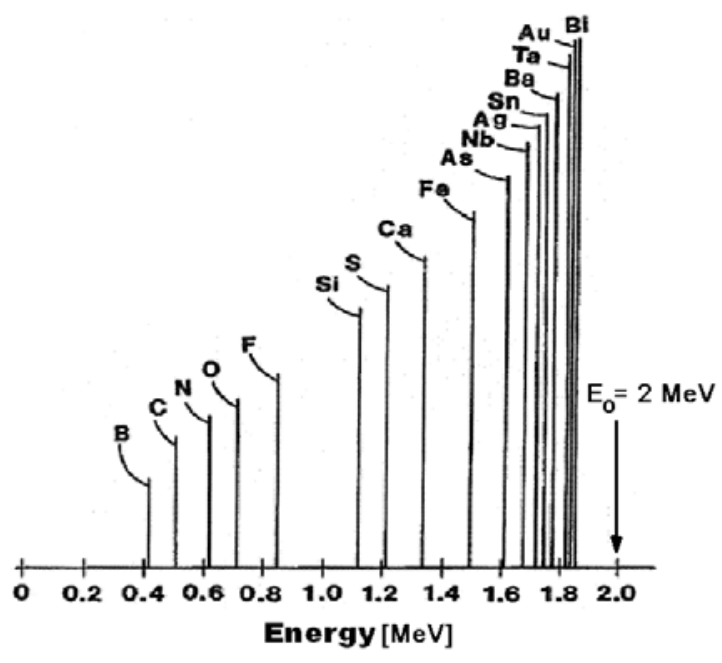

Figure 3.2.1 Mass dependence of the $\mathrm{k}$ factor (illustration of equation 3.2.1): $\mathrm{m}=4, \mathrm{E}_{\mathrm{o}}=2$ $\mathrm{MeV}$ and $\theta=180^{\circ}$.
Hence, measuring the energy of the backscattered particle provides information on mass $M_{i}$. This equation, for the alpha particles $(m=4)$ with the incident energy $E_{0}=2 \mathrm{MeV}$ and the scattering angle $\theta=180^{\circ}$, is illustrated in Figure 3.2.1. In this case equation 3.2.1 gets the form: $K_{i}=\left[\left(M_{i}-4\right) /\left(M_{i}+\right.\right.$ $4)]^{2}$. When increasing the mass of the target nucleus $M_{i}$, the measured energy $E_{i}$ increases, and the difference between the adjacent values of $E_{i}$ decreases. 


\section{Cross-section and backscattering yield}

When more than one type of nuclei is present in the sample, the relative intensity of the corresponding signals depends on their concentrations through the known differential cross-sections for Rutherford scattering into the solid angle $d \Omega$ :

$$
\frac{d \sigma_{\mathrm{i}}}{d \Omega}=\left(\frac{\mathrm{Z}_{\mathrm{m}} \cdot \mathrm{Z}_{\mathrm{i}} \cdot e^{2}}{4 \mathrm{E}_{0}}\right)^{2} \cdot \frac{4}{\sin ^{4} \theta} \cdot \frac{\left\{\left[1-\left(\mathrm{m} / \mathrm{M}_{\mathrm{i}}\right)^{2} \cdot \sin ^{2} \theta\right]^{1 / 2}+\cos \theta\right\}^{2}}{\left[1-\left(\mathrm{m} / \mathrm{M}_{\mathrm{i}}\right)^{2} \cdot \sin ^{2} \theta\right]^{1 / 2}}
$$

For increasing target charge $Z_{i}$, the cross section increases quadratically. Hence, for heavy masses the backscattered signal is much higher than that of lower mass nuclei. The number of detected backscattered particles $N_{i}$ is given by:

$$
N_{i}=\frac{d \sigma}{d \Omega} \cdot d \Omega \cdot Q \cdot N \cdot D_{E f}
$$

where $Q$ is the total number of the incident particles, $N$ the concentration of the target atoms and $D_{E f}$ the efficiency of the detector system.

\section{Energy loss and depth scale}

Light energetic incident particles, in most cases of RBS these are alpha particles, lose energy when penetrating the material, which is mainly due to electronic stopping. In backscattering spectrometry, where the elastic collision takes place at the depth $d$, one considers the energy loss along the inward and on the outward path (see Fig. 3.2.2). So, when a particle is scattered by a nucleus located at a certain depth $d$, its energy will already be reduced before the collision. Thus, the energy of the backscattered particles can be written as:

$$
E_{i}=K_{i} \cdot\left(E_{o}-\Delta E(d)\right)-\Delta E(d / \cos \theta) \quad(3.2 .3)
$$

where $\Delta E(d)$ and $\Delta E(d / \cos \theta)$ are the energy loss. The depth scales are determined from energy loss values, which are given in following ref [3-4, 5]. Here, we neglected the energy dependence of $\Delta E(d)$ and $\Delta E(d / \cos \theta)$ during both paths in and out. 


\section{Kinematic Factor}

$$
\mathbf{K}=\frac{\mathbf{E}_{\text {after }}^{\text {scattering }}}{\mathbf{E}_{\text {before }}^{\text {scattering }}}
$$

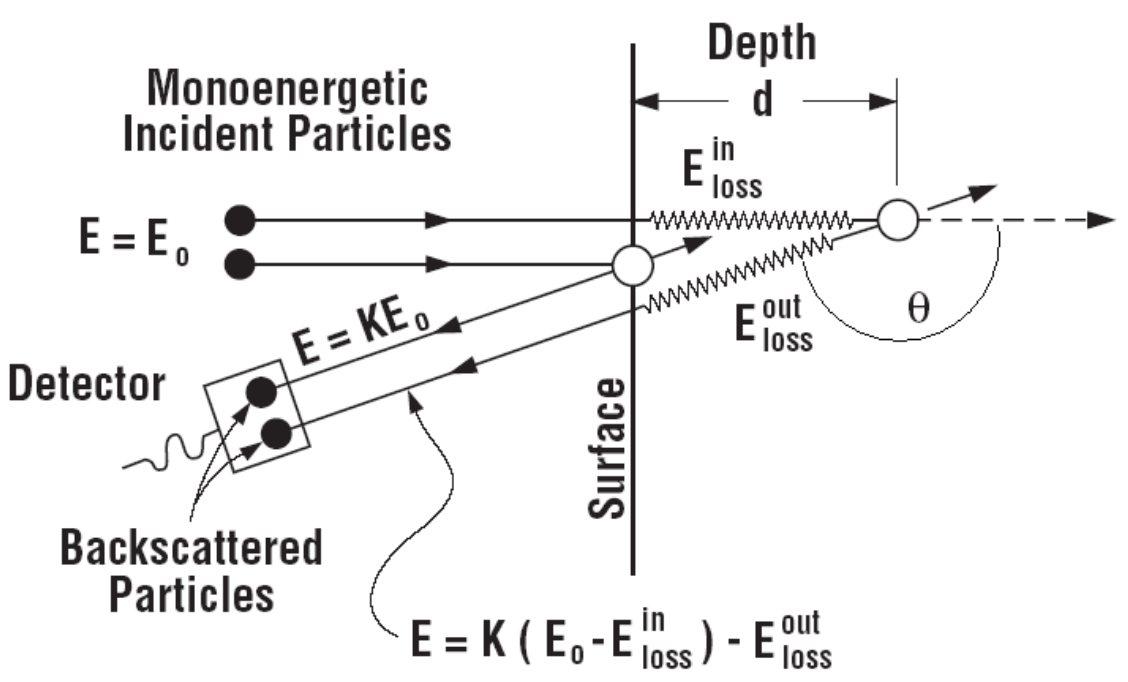

Figure 3.2.2 Schematic representation of energy loss.

All the discussed topics related to RBS can be found in the literature: [3-6, 7, 8], to name only a few of the titles.

Several RBS data analysis software packages are available, such as: RUMP [3-9], NDF [3-10] or IBA [3-11]. In this work RUMP and WiNDF (Windows version of NDF) have been used.

In the present RBS experiments, a $900 \mathrm{keV} \alpha$ - particle beam from the multipurpose IONAS accelerator $[3-8,12]$ was used in most cases. The scattered particles were detected at $\theta=165^{\circ}$ to the beam. For the detection of the particles, two silicon surface detectors with a typically energy resolution of 12 - $13 \mathrm{keV}$ (FWHM) and a solid angle $d \Omega$ of 3.2 msr were used. A few of the RBS experiments were performed at the University of Jena with a $1 \mathrm{MeV} \alpha$ - particle beam, with a silicon surface detector positioned at $\theta=168^{\circ}$, and an energy resolution and solid angle of $15 \mathrm{keV}$ and 3.2 msr, respectively. 
As an example, a raw RBS spectrum and a fit of it, as well as the corresponding deduced depth profiles are shown in Figure 3.2.3. This thin film consists of two layers: a $20 \mathrm{~nm}$ thick layer of ${ }^{57}$ Fe deposited on a (111) Si wafer, and a $45 \mathrm{~nm}$ thick layer of natural iron deposited on top of the ${ }^{57} \mathrm{Fe}$ layer. The fitting procedure was performed with the WiNDF code. In order to carry out a fit of the raw spectra, five sublayers were introduced. Each of them has a different thickness and composition, as can be seen in Table 3.2.1. For converting the at $/ \mathrm{cm}^{2}$ to the depth unit - nm, an average
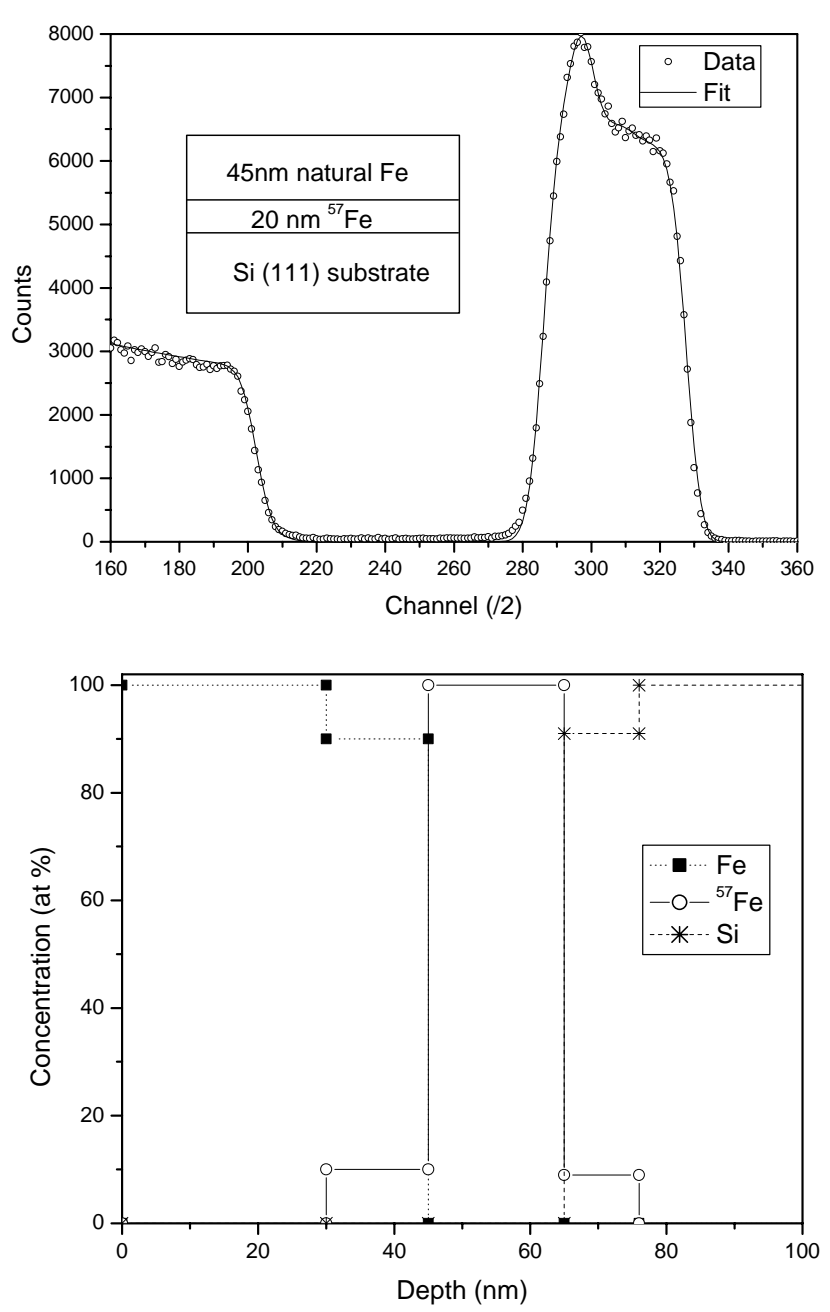

Figure 3.2.3 Example of the RBS spectra: $45 \mathrm{~nm}$ natural $\mathrm{Fe} / 20 \mathrm{~nm}{ }^{57} \mathrm{Fe} / \mathrm{Si}$ (111).

density of the two

components was assumed.

Table 3.2.1 Thickness and composition of the sublayers used for the fitting procedure of the raw spectra shown in Fig. 3.2.3.

\begin{tabular}{|c|c|c|c|c|}
\hline Layer & Thickness (nm) & Atomic \% of ${ }^{\text {nat. }} \mathrm{Fe}$ & Atomic \% of ${ }^{57} \mathrm{Fe}$ & Atomic \% of Si \\
\hline 1 & 30 & 100 & 0 & 0 \\
\hline 2 & 17 & 90 & 10 & 0 \\
\hline 3 & 18 & 0 & 100 & 0 \\
\hline 4 & 11 & 0 & 9 & 91 \\
\hline 5 & bulk & 0 & 0 & 100 \\
\hline
\end{tabular}


Some of the advantages of RBS have already been mentioned, but there are also a few disadvantages. Here, two of them will be discussed briefly:

Fundamental unit of concentration obtained by RBS is in atoms $/ \mathrm{cm}^{2}$ and the concentration of the various atoms in the sample versus the backscattering energy loss. In order to convert a backscattering spectrum into a depth profile (nm unit) it is necessary to assume a density for the sample. In the case of single-element and compound films, the bulk density can be assumed for the film, and the thickness is obtained with nm accuracy. In the case of multielemental ("mixed") films with an unknown density, one may calculate the depth-dependent density of each element normalized to its concentration and incorporate this result into the WiNDF analysis. Since the RBS technique only depends on Couloumb scattering from the nuclei, in first order, it does not reveal any information on the electronic structure or chemical bonding. Implications of stoichiometric compound formation deduced from the sample composition must always be verified by other techniques, such as Mössbauer spectroscopy or X-ray diffraction.

\subsection{Mössbauer spectroscopy}

Mössbauer spectroscopy is based on the recoilless nuclear resonance emission and absorption of gamma rays by nuclei in solids. Its energy resolution of $\sim 10^{-12}$ is sufficient to resolve the hyperfine structures of nuclear levels, thereby making it the most precise physical measurement ever achieved. In a Mössbauer experiment only one type of isotopes is affected by the gamma-ray transition between the ground state and a specific excited state, and no other elements/isotopes contribute to the Mössbauer spectra. The traditional source of gamma rays (synchrotron radiation is "not traditional") is a radioactive source that decays into the aimed Mössbauer isotope with the following properties: long radioactive $\beta$-decay half-life (allows time to prepare and use the setup), large Mössbauer recoilless fraction for emission (DebyeWaller factor; most of the emitted gamma rays are not recoil-broadened), single-lined (without hyperfine splittings), small (intrinsic) resonance width of the transition. The intrinsic linewidth (i.e. $\Gamma$, or full width at a half maximum - FWHM) is a property of the particular transition (not depending on the host material) and is given by the relation:

$$
\Gamma=\hbar / \tau
$$

where $\tau$ is the lifetime of the excited nuclear state. 
Many isotopes have these properties, such as ${ }^{119} \mathrm{Sn},{ }^{151} \mathrm{Eu},{ }^{129} \mathrm{I}$ (see Figure 3.3.1.), but still the isotope ${ }^{57} \mathrm{Fe}$, with its $2.2 \%$ of abundance in natural iron, is the most common Mössbauer isotope. More than 95\% of all Mössbauer measurements are performed on this isotope.

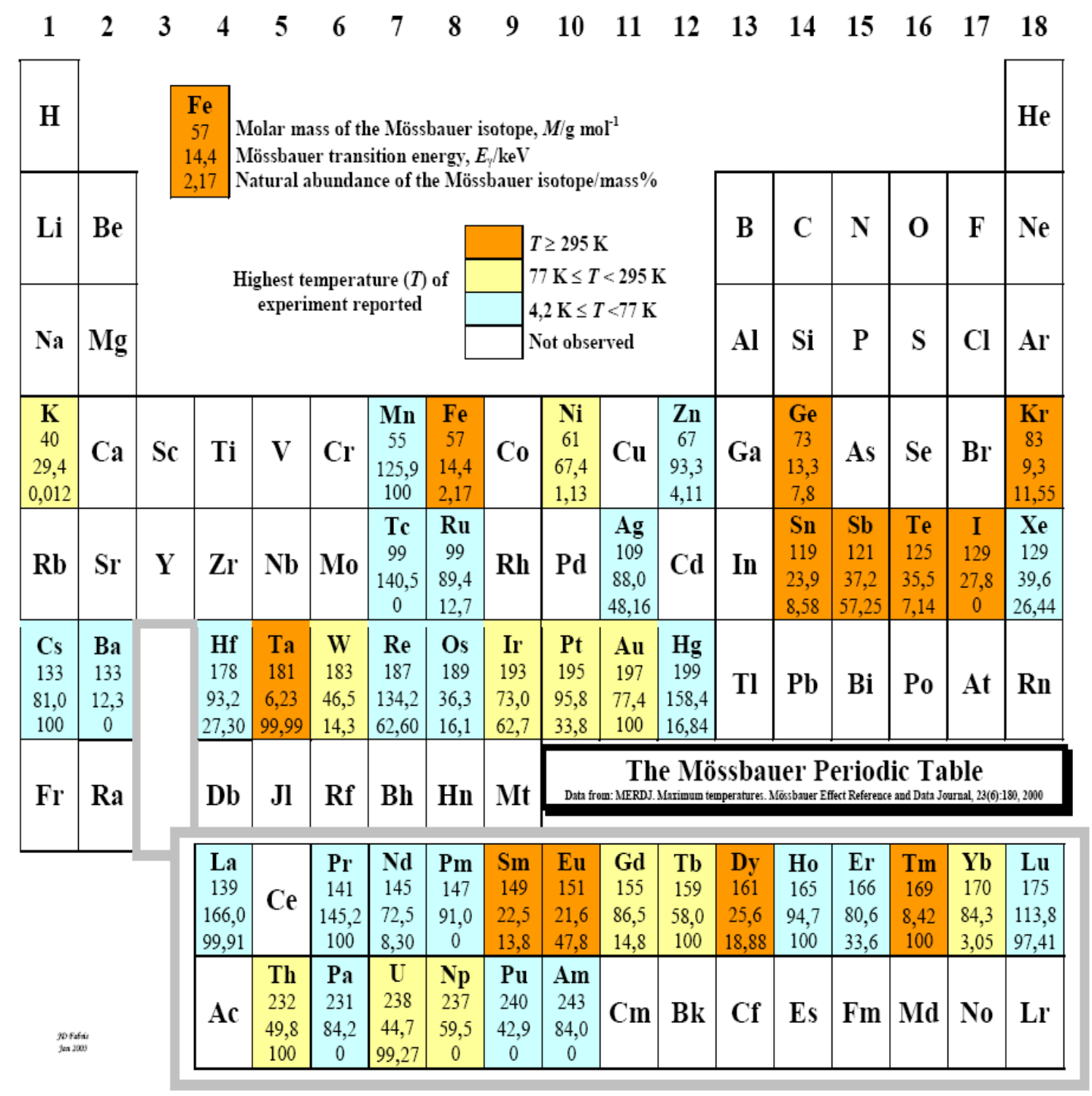

Figure 3.3.1 List of isotopes suitable for Mössbauer spectroscopy. 
The decay of ${ }^{57} \mathrm{Fe}$ from its parent, ${ }^{57} \mathrm{Co}$, is shown in Figure 3.3.2. The $14.4 \mathrm{keV} \gamma$-ray is emitted from the excited state of ${ }^{57} \mathrm{Fe}$ via magnetic dipole transition from the $\mathrm{I}=3 / 2$ metastable state to the $\mathrm{I}=1 / 2$ ground state. The linewidth of the emitted gamma ray is only $4.7 \times 10^{-9} \mathrm{eV}$.

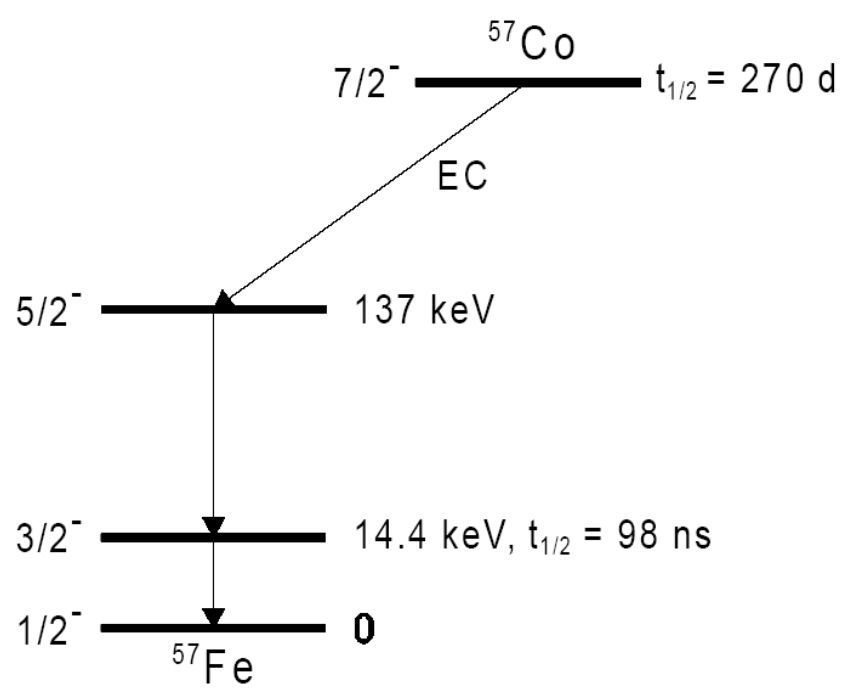

Figure 3.3.2 The decay scheme of ${ }^{57} \mathrm{Co}$.

\section{Apparatus}

The ${ }^{57}$ Fe nuclei are embedded in two solids; those in the source, which originate from the ${ }^{57} \mathrm{Co}$ decay, and those in the sample to be tested, which are in the ground state. In our case, ${ }^{57} \mathrm{Co}$ in an fcc-Rh matrix is used as a source, and the conversion electrons of the $14.4 \mathrm{keV}$ transition are detected (Conversion Electron Mössbauer Spectroscopy CEMS). The conversion electrons escape from the upper $150 \mathrm{~nm}$ of the sample, and in this way only provide information on thin film. In the present experiment, a triple Mössbauer spectrometer was used [3-13], but no data from transmission and conversion X-ray Mössbauer spectroscopy were recorded. The radioactive ${ }^{57} \mathrm{Co}$ source is mounted on an electromechanical drive that moves with a well-defined velocity $v$. Generally, velocities of a few $\mathrm{mm} / \mathrm{s}$ are required. The energy of the emitted gamma rays is shifted, due to the Doppler effect, by an amount proportional to the velocity. The obtained spectrum is given in units of the Doppler velocity $v$, which is related to the energy shift $\Delta E$ via:

$$
\Delta E=(v / c) \cdot E_{\gamma}
$$

where $c$ is the speed of light and $E_{\gamma}$ the Mössbauer transition energy (14.4 keV for ${ }^{57} \mathrm{Fe}$ ). The conversion (and Auger) electrons were detected in a flat $2 \pi \mathrm{He} / \mathrm{CH}_{4}$ gasflow proportional counter. In order to reduce the background, the amplified signals 
from the detector were separated by an energy discriminator and then stored in a multichannel analyzer with 1024 channels.

\section{Hyperfine interactions}

There are three important hyperfine interactions detected by means of Mössbauer spectroscopy: the isomer shift, which differentiates between the ionic valence such as $\mathrm{Fe}^{2+}$ or $\mathrm{Fe}^{3+}$ and the electron density at the site of the nucleus; electric quadrupole splitting, which reflects the lattice asymmetry; and nuclear Zeeman splitting, which measures the ferromagnetic hyperfine field.

a) The simplest hyperfine interaction is the electric monopole interaction. This is due to the shielding of electrons that find themselves inside the nuclear volume. Since the nuclear site can be different for different nuclear states, the resulting energy shifts depend on the corresponding nuclear levels. The electric monopole interaction does not split the levels, but only affects their precise energies. The corresponding Mössbauer resonance is a single line called "singlet". Whenever the source and the sample have different electron densities at the nuclear site, the observed absorption occurs at some non-zero Doppler velocity. The isomer shift (IS) of the absorber relative to the source is given by:

$$
\text { IS }=\text { const. } \cdot\left(\left(R^{*}\right)^{2}-R^{2}\right) \cdot\left[\rho_{a}(0)-\rho_{s}(0)\right] \quad(3.3 .3),
$$

where $R^{*}$ and $R$ are the mean nuclear radii in the excited and ground state, respectively, and $\rho_{a}(0)$ and $\rho_{s}(0)$ are the total electron densities at the nuclei in the absorber and the source. IS can also be defined relative to a chosen calibration material, in which case $\rho_{s}(0)$ is the electron density at the probe nuclei of the standard. In all the CEMS measurements of this work the IS are given in comparison to $\alpha$-Fe. Since only s-orbitals have a finite electron density at the nucleus, only they contribute to $\rho_{a}(0)$ and $\rho_{s}(0)$. In addition, s-type conduction electrons in metals can also contribute.

b) The electric quadrupole interaction is the hyperfine interaction between the electric quadrupole moment of the nucleus, $e Q$, and the local electric field gradient (EFG). The EFG is caused by the non-spherical distribution of the electrons around the probe nuclei via long range Coulomb electric forces. The EFG is a tensor described by the three componenets $V_{z z}, V_{x x}$ and $V_{y y}$ in the principal-axes coordinate system. As $V_{x x}+$ $V_{y y}+V_{z z}=0$, the EFG can be expressed by two quantities: the maximum component $V_{z z}$ and the asymmetry parameter: 


$$
\eta=\left(V_{x x}-V_{y y}\right) / V_{z z} \quad \text { (3.3.4). }
$$

This interaction partially removes the degeneracies of the nuclear states. As the ${ }^{57} \mathrm{Fe}$ ground state has no quadrupole moment $(I=1 / 2)$, it is not affected by the EFG interaction. But the first excited state $(I=3 / 2)$ has a quadrupole moment and the degeneracy of its magnetic substates will be partially removed in the presence of an EFG. The excited state is split into two magnetic substates with magnetic quantum numbers $m= \pm 1 / 2$ and $m= \pm 3 / 2$. The splitting is called quadrupole splitting (QS, or $\Delta$ ), and is given by:

$$
\Delta=\left(e^{2} q Q / 2\right) \cdot\left[1+\eta^{2} / 3\right]^{1 / 2} \quad(3.3 .5)
$$

Whenever $V_{z z} \neq 0$, the Mössbauer resonance in ${ }^{57} \mathrm{Fe}$ consists of a doublet. The relative intensities of the two lines depend on the orientation of the EFG tensor relative to the incident gamma ray direction. For a sample in which all directions are uniformly distributed, the two lines have equal intensities (see Fig 3.3.3).

c) The magnetic dipole, or hyperfine field, interaction is the interaction between the nuclear magnetic moment and the effective magnetic field that is felt by the nucleus. This interaction completely removes all the magnetic substate degeneracy and produces a "Zeeman splitting" for the ground state (two substates with $m= \pm 1 / 2$ ) and the first excited state (four substates with $m= \pm 1 / 2, \pm 3 / 2$ ), and it is equal to:

$$
\Delta E=g^{*} \mu_{N} B_{h f}
$$

where $g^{*}$ is the nuclear $g$-factor of the considered state, $\mu_{N}$ is the nuclear magneton, and $B_{h f}$ is the hyperfine field. Due to the splittings of the ground and excited state, resonance absorption can occur for six possible combinations, connected by dipole transitions. The resonance consists of six lines called "sextet”.

All three hyperfine interactions are presented in Figure 3.3.3.

Literature about the Mössbauer effect can be traced since the first original work of Mössbauer [3-14], until present days [3-15, 16], which shows constant development and improvement of the Mössbauer spectroscopy. 


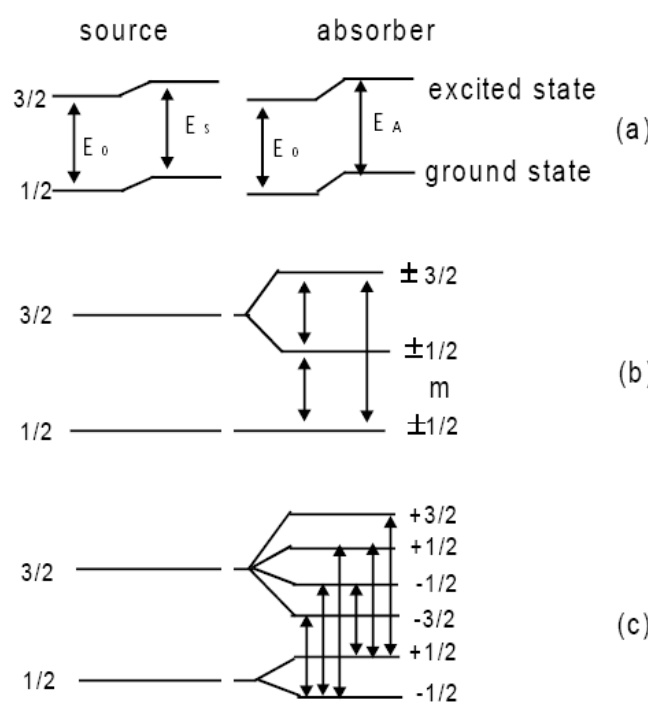

(a)

(b)

(c)

Figure 3.3.3 Schematic presentation of the energy levels and three hyperfine interactions: a) electric monopole interaction, IS b) electric quadrupole interaction, QS c)magnetic hyperfine interaction, $\mathrm{H}$.

In order to fit the raw CEMS spectrum, least-square routines were used by superimposing the Lorentzian lines. The fitting procedure was performed with the Recoil program package [3-17]. The hyperfine parameters used for fitting the different phases observed in the CEMS spectrum are listed in Table 3.3.1.

Table 3.3.1 Hyperfine parameters used for fitting the CEMS spectra. $\left(\mathrm{B}_{\mathrm{hf}}=\right.$ magnetic hyperfine field, IS = isomer shift, $\Delta=$ quadrupole splitting, $\mathrm{RA}=$ relative area of the subspectrum.)

\begin{tabular}{|c|c|c|c|c|c|}
\hline Phase & $\mathrm{B}_{\mathrm{hf}}(\mathrm{T})$ & IS $(\mathrm{mm} / \mathrm{s})$ & $\Delta(\mathrm{mm} / \mathrm{s})$ & RA (\%) & Ref. \\
\hline$\alpha-\mathrm{Fe}$ & 33 & 0 & 0 & 100 & \multirow{19}{*}{$\begin{array}{c}\text { [3-18, 19, } \\
20] \\
\text { and } \\
\text { references } \\
\text { therein }\end{array}$} \\
\hline$\alpha^{\prime}-\mathrm{Fe}(\mathrm{N})$ & 30.4 & 0.08 & & & \\
\hline \multicolumn{5}{|l|}{$\varepsilon-\mathrm{Fe}_{2+\mathrm{x}} \mathrm{N}$} & \\
\hline \multirow{2}{*}{$\mathrm{x}=1.2$} & 29.8 & 0.24 & & 12 & \\
\hline & 23.8 & 0.33 & & 88 & \\
\hline \multirow{3}{*}{$x=1.0$} & 28.57 & 0.21 & & 11.5 & \\
\hline & 21.83 & 0.33 & & 77.0 & \\
\hline & 7.0 & & & 11.5 & \\
\hline \multirow{3}{*}{$x=0.67$} & 27.4 & 0.26 & & $\approx 3$ & \\
\hline & 20.50 & 0.34 & & 61 & \\
\hline & 9.95 & 0.40 & & 36 & \\
\hline \multirow{2}{*}{$x=0.47$} & 18.6 & 0.35 & & 52 & \\
\hline & 8.4 & 0.41 & & 48 & \\
\hline \multirow{3}{*}{$\gamma^{\prime}-\mathrm{Fe}_{4} \mathrm{~N}$} & 34.06 & 0.24 & 0.00 & 25 & \\
\hline & 21.55 & 0.3 & 0.22 & 50 & \\
\hline & 21.92 & 0.3 & 0.43 & 25 & \\
\hline \multirow{2}{*}{$\beta-\mathrm{FeSi}_{2}$} & - & 0.09 & 0.54 & 50 & \\
\hline & - & 0.10 & 0.323 & 50 & \\
\hline amorph. Fe-Si & & 0.2 & 0.7 & & \\
\hline
\end{tabular}


The raw CEMS spectrum and its deconvolution are shown in Figure 3.3.4. The hyperfine parameters used in the fitting process are listed in Table 3.3.1.

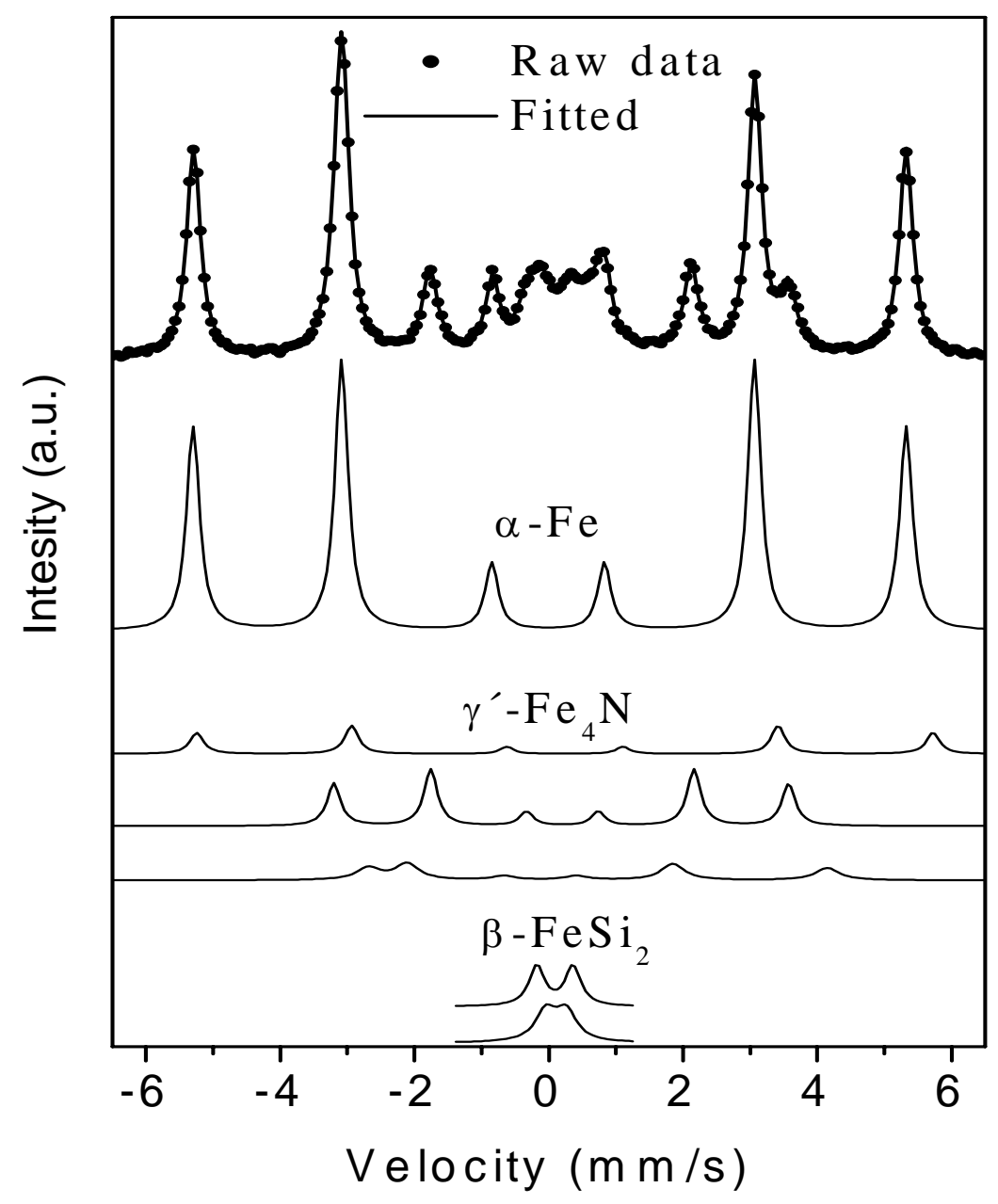

Figure 3.3.4 Deconvoluted CEM spectrum of a Fe/Si sample irradiated to the fluence of $1 \mathrm{x} \cdot 10^{17} \mathrm{~N} / \mathrm{cm}^{2}$ and annealed at $600^{\circ} \mathrm{C}$. The subspectra corresponding to $\alpha-\mathrm{Fe}, \gamma^{\prime}-\mathrm{Fe}_{4} \mathrm{~N}$ and $\beta-\mathrm{FeSi}_{2}$ are indicated with the hyperfine parameters listed in Table 3.3.1. 
Magnetic Orientation Mössbauer Spectroscopy (MOMS)

The intensities of transitions between the magnetic substates depend on the ClebschGordon coefficients of the particular transition [3-21]. Table 3.3.2 presents their values for ferromagnetic spectra in the case of ${ }^{57} \mathrm{Fe}$ :

Table 3.3.2 Intensities of the magnetic transitions in the ${ }^{57} \mathrm{Fe}$ isotope. $\Delta \mathrm{m}$ is the difference in the magnetic quantum numbers between two energy states, $\theta$ is the angle between the incident $\gamma$-ray and the principal axis of the magnetic field or the EFG tensor.

\begin{tabular}{|c|c|c|c|}
\hline Line & Transition & $\Delta \mathrm{m}$ & Intensity \\
\hline L1 & $-3 / 2 \rightarrow-1 / 2$ & +1 & $\mathrm{I}_{1}=3 / 8\left(1+\cos ^{2} \theta\right)$ \\
\hline L2 & $-1 / 2 \rightarrow-1 / 2$ & 0 & $\mathrm{I}_{2}=1 / 2\left(1-\cos ^{2} \theta\right)$ \\
\hline L3 & $+1 / 2 \rightarrow-1 / 2$ & -1 & $\mathrm{I}_{3}=1 / 8\left(1+\cos ^{2} \theta\right)$ \\
\hline L4 & $-1 / 2 \rightarrow+1 / 2$ & +1 & $\mathrm{I}_{4}=1 / 8\left(1+\cos ^{2} \theta\right)$ \\
\hline L5 & $+1 / 2 \rightarrow+1 / 2$ & 0 & $\mathrm{I}_{5}=1 / 2\left(1-\cos ^{2} \theta\right)$ \\
\hline L6 & $+3 / 2 \rightarrow+1 / 2$ & -1 & $\mathrm{I}_{6}=3 / 8\left(1+\cos ^{2} \theta\right)$ \\
\hline
\end{tabular}

If the hyperfine field vector is in the plane of the thin film, and if the angle between the $\gamma$-ray and the sample normal is $0^{\circ}\left(\theta=90^{\circ}\right)$, the ratio of the relative intensities is:

$$
\mathrm{I}_{1}: \mathrm{I}_{2}: \mathrm{I}_{3}=3: 4: 1
$$

If the sample is then rotated around the direction of the sample normal, the angle $\theta$ remains unchanged and the relative intensities do not change. When the geometry of the experiment is changed in such a way that the $\gamma$-ray does not enter along the surface of the sample normal, the relative intensity ratio $\mathrm{I}_{1}, \mathrm{I}_{2}, \mathrm{I}_{3}$ depends on their angle, while the ratio $I_{1}: I_{3}$ ratio remains constant. In our work we have measured the changes in the relative intensities between the sextet lines 2 and 3, which are given by the following equation [3-22, 23, 24]:

$$
\frac{I_{2}}{I_{3}}(\varphi)=4 \cdot \sum_{i=1}^{n} c_{i} \cdot \frac{1-\sin ^{2} \alpha \cdot \sin ^{2}\left(\varphi-\psi_{i}\right)}{1+\sin ^{2} \alpha \cdot \sin ^{2}\left(\varphi-\psi_{i}\right)}+4 \cdot\left(1-\sum_{i=1}^{n} c_{i}\right) \cdot \frac{1-\cos ^{2} \alpha}{1+\cos ^{2} \alpha}
$$

where $\alpha$ is the angle between the $\gamma$-ray and the surface normal, $\varphi$ is the rotation angle of the sample around its normal, measured from an arbitrarily chosen zero direction. The $\Psi_{i}$ are the directions of the in-plane vectors of the magnetization, and $c_{i}$ their relative contributions. If all hyperfine field vectors are in the film plane (i.e. $\mathrm{I}_{1}: \mathrm{I}_{2}: \mathrm{I}_{3}$ 
$=3: 4: 1$ ), then $\sum_{i=1}^{n} c_{i}=1$, and the second term in equation 3.3.8 disappears. Finally, if the sample is tilted by $\alpha=45^{\circ}$, equation 3.3.8 becomes:

$$
\frac{I_{2}}{I_{3}}(\varphi)=4 \cdot \sum_{i=1}^{n} c_{i} \cdot \frac{1-0.5 \cdot \sin ^{2}\left(\varphi-\psi_{i}\right)}{1+0.5 \cdot \sin ^{2}\left(\varphi-\psi_{i}\right)}
$$

The typical MOMS data are shown in the next few figures. These measurements were performed on the sample, which contains a $20 \mathrm{~nm}$ thick layer of ${ }^{57} \mathrm{Fe}$ deposited on a (111) Si wafer and covered by a $45 \mathrm{~nm}$ thick layer of natural iron. In order to check if all hyperfine vectors are in the film plane, first a CEMS measurement was done with the $\gamma$-rays perpendicular to the surface of the sample (Fig. 3.3.5). The spectrum was fitted with the hyperfine parameters of the $\alpha$-iron, with a relative intensity ratio of: $\mathrm{I}_{1}$ $: \mathrm{I}_{2}: \mathrm{I}_{3}=3: 4: 1$.

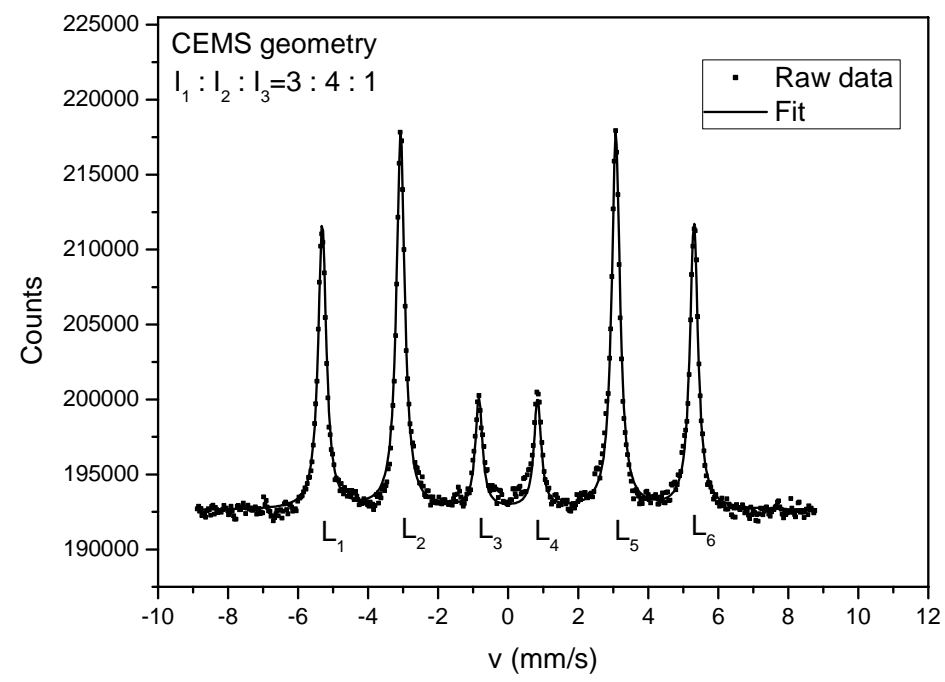

Figure 3.3.5 CEMS spectrum and coresponding fit of 45nm $\mathrm{Fe} / 20 \mathrm{~nm}^{57} \mathrm{Fe} / \mathrm{Si}$.

Then, the sample was tilted by $\alpha=45^{\circ}$ and the spectra were taken at intervals of $\Delta \varphi=$ $20^{\circ}$. The spectra taken at $\varphi=120^{\circ}$ and $\varphi=240^{\circ}$ are shown in Figure 3.3.6. Finally, a fit of the measured $I_{2} / I_{3}(\varphi)$ ratios is shown in Figure 3.3.7. Three terms of equation 3.3.9 were necessary in order to get a proper fit of the data: $c_{1}=0.35(4), c_{2}=0.26(3)$ and $c_{3}=0.40(4)$ with corresponding angles of $\Psi_{1}=81(12)^{\circ}, \Psi_{2}=204(13)^{\circ}$ and $\Psi_{3}=$ $310(11)^{\circ}$. Almost equal contribution of each vector, which are approximately 
separated by $120^{\circ}$, indicate that the hyperfine field is almost randomly distributed inplane.

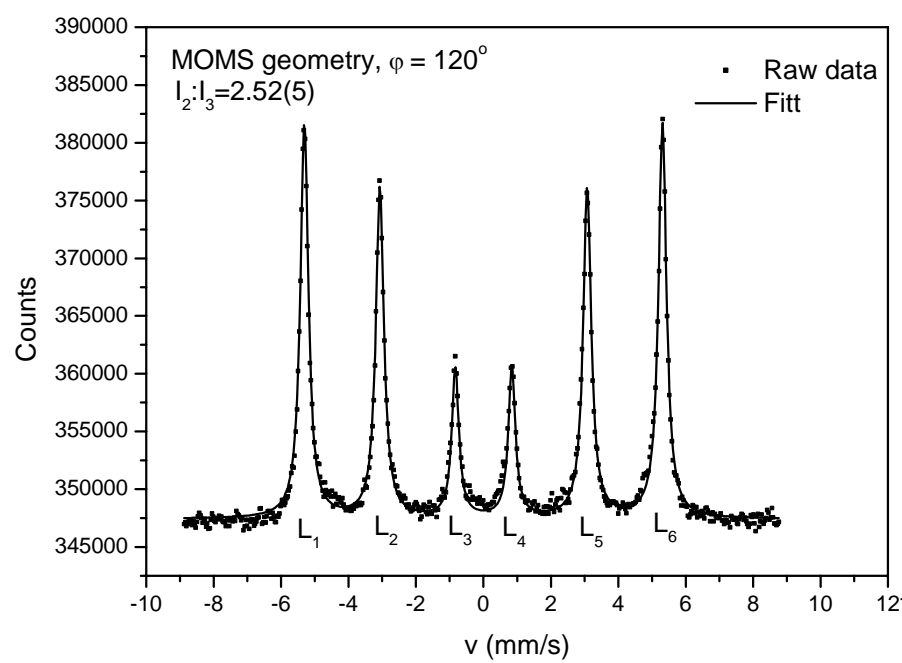

(a)

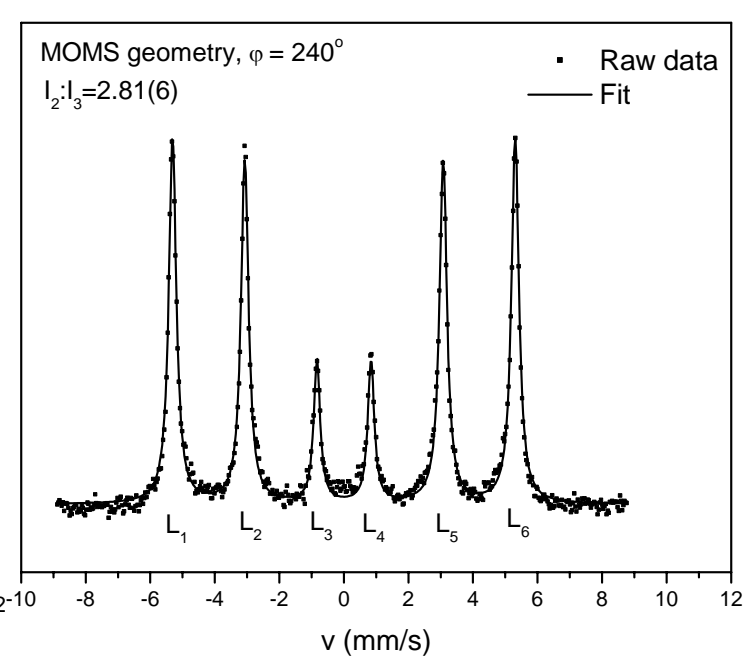

(b)

Figure 3.3.6 MOMS spectra $\left(\alpha=45^{\circ}\right)$ and corresponding fits for a $45 \mathrm{~nm} \mathrm{Fe} / 20 \mathrm{~nm}^{57} \mathrm{Fe} / \mathrm{Si}$ sample taken at $\varphi=120^{\circ}$ (a) and $240^{\circ}(\mathrm{b})$. The corresponding $\mathrm{I}_{2} / \mathrm{I}_{3}$ ratios are 2.52(5) and 2.81(6), respectively.

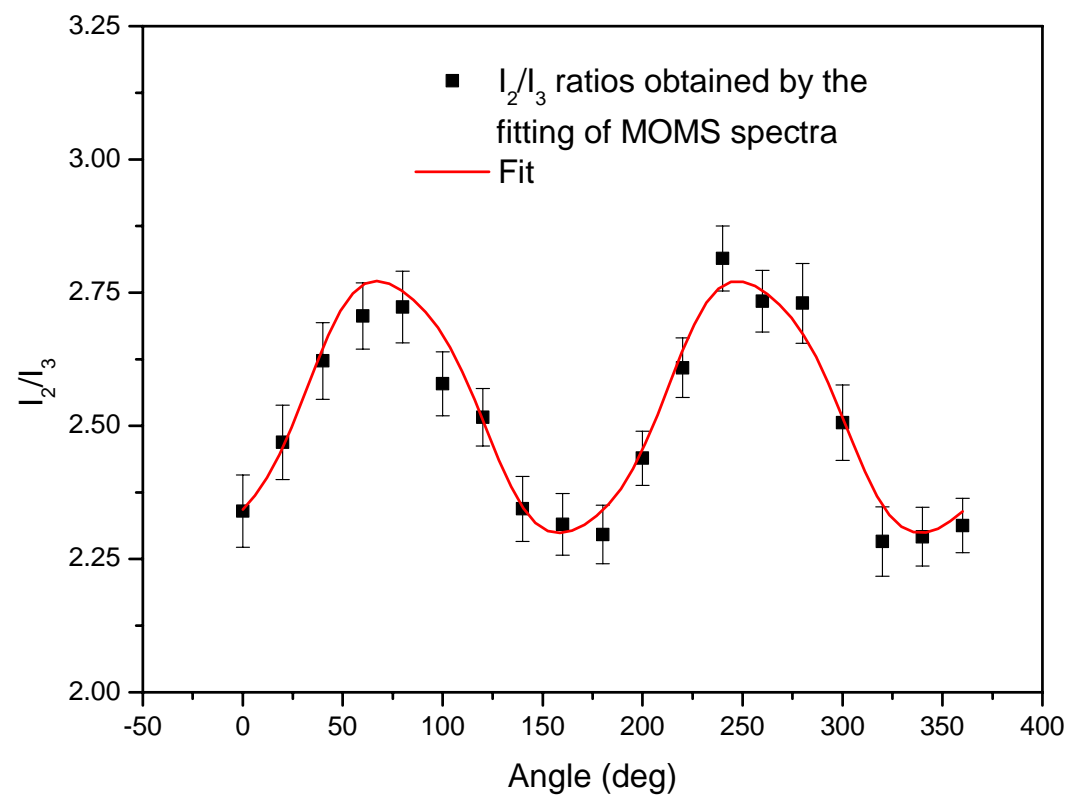

Figure 3.3.7 Relative ratios of $\mathrm{I}_{2} / \mathrm{I}_{3}(\varphi)$, and corresponding fit. The components of the fit are: $c_{1}=0.35(4), c_{2}=0.26(3)$ and $c_{3}=0.40(4)$, with corresponding angles of $\Psi_{1}=81(12), \Psi_{2}=204(13)$ and $\Psi_{3}=310(11)$. 


\subsection{The Magneto-Optical Kerr Effect (MOKE)}

Magneto optics describes the interaction of electromagnetic radiation with magnetized matter. Magneto-optical effects are observed in a wide energy range, from microwaves through X-rays. The Faraday effect and the magneto-optical Kerr effect (MOKE) are induced by visible light, and they are linear functions of the magnetization $\mathbf{M}$. The Faraday effect is the change in polarization that occurs upon transmission of linearly polarized light through a magnetized sample with the magnetization perpendicular to the surface [3-25]. MOKE can be observed as a change in the intensity and/or polarization of light reflected from a magnetized sample as a function of the applied magnetic field [3-26]. The Kerr signal is induced by a specific magnetization component, which is determined by the experimental geometry used. Three geometries of the MOKE effect can be distinguished (Fig3.4.1): polar, longitudinal and transversal.

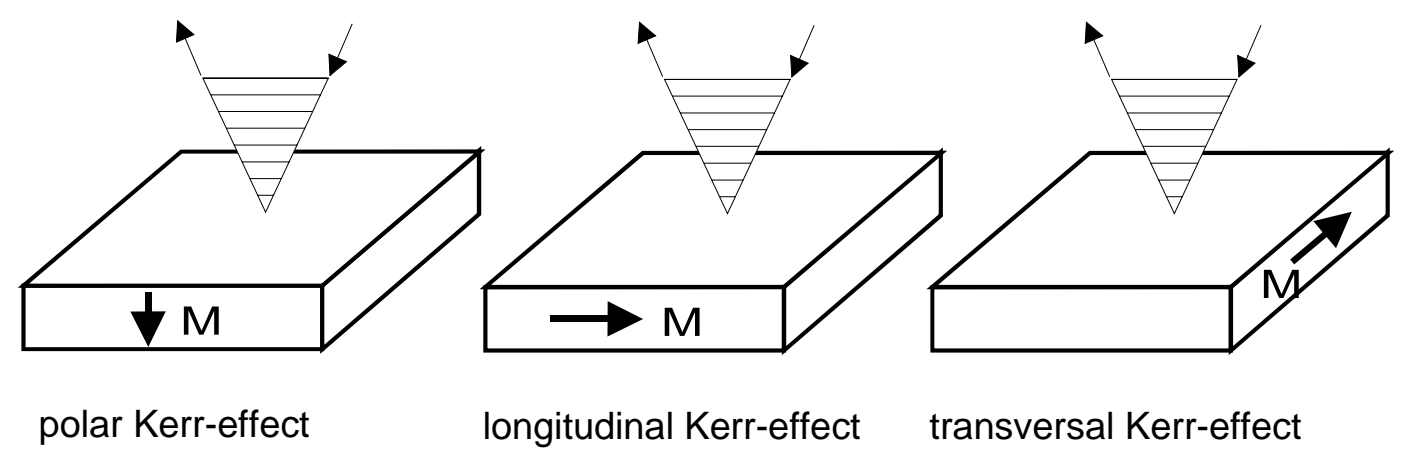

Figure 3.4.1 MOKE geometries: out-of-plane magnetization (polar); in plane magnetization with $\mathbf{M}$ parallel (longitudinal) or perpendicular (transversal) to the plane of incidence light.

For conventional MOKE measurements, the light reflected by the magnetized sample passes through an analyzing polarizer (the analyzer) and onto a photodetector, providing the signal. The nature of the Kerr effect depends on the magnetization (M) with respect to the scattering plane and the sample plane. When $\mathbf{M}$ is in the scattering plane and the sample plane (longitudinal orientation) or when $\mathbf{M}$ is in the scattering plane but perpendicular to the sample plane (polar orientation), the Kerr effect produces a change in the polarization state of the reflected light and the analyzer is set close to extinction in order to observe a change in the polarization state. When $\mathbf{M}$ is perpendicular to the scattering plane but in the same plane (transverse orientation), the Kerr effect is seen only as a change in the intensity of the reflected beam and the analyzer is set to transmit light of the same polarization as the incident light. 
As mentioned earlier, when linearly polarized light is reflected by a magnetic film, its polarization becomes elliptic (Kerr ellipticity: $\epsilon_{\text {Kerr }}$ ) and the principal axis is rotated (Kerr rotation: $\phi_{\text {Kerr }}$ ). Both effects depend on the off-diagonal component of the conductivity tensor, polarizability tensor and frequency, and they are linear functions of $\mathbf{M}$.

Detailed expressions for these two effects, obtained by the quantum-mechanical approach, can be found in the following references: [3-27, 28, 29] In the present work, the longitudinal Kerr effect was used. Fig. 3.4.2 shows the MOKE set-up used in all our measurements [3-24].

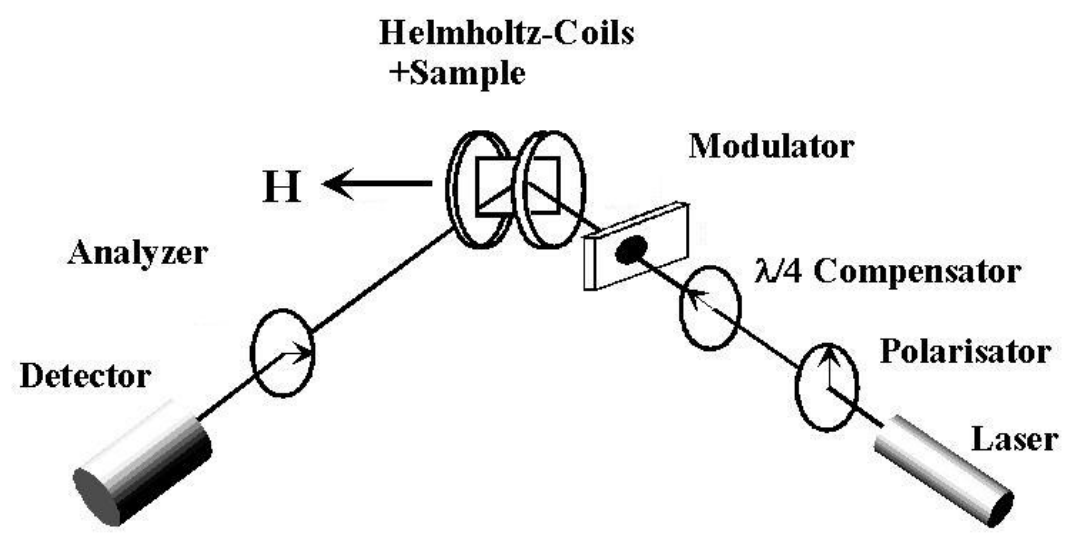

Figure 3.4.2 Schematic drawing of the MOKE set-up.

As a light source, a He-Ne laser with a power of $2 \mathrm{~mW}$ and a wavelength of $\lambda=632.8$ $\mathrm{nm}$ was used. To adjust the polarity of the laser light, a polarisator was placed directly in the front of a laser. The polarization axis was set at $90^{\circ}$ relative to the incidence plane of the light on the sample. Behind the polarisator, the light beam passed through a $\lambda / 4$ compensator which delayed the component parallel to the slow axis of light by $\pi / 2$. Before hitting the sample, the beam passed through a modulator, which changed the phase of the horizontal component of the light with a modulation frequency of 50 $\mathrm{kHz}$, and the previously circular polarized light then showed a modulated linear and circular polarization. The linear polarization was oriented at $\pi / 4$ after $T / 4$ or at $3 \pi / 4$ after 3T/4. And the light hit the sample, which was placed in a goniometer, inside water-cooled Helmholtz coils, which provided a field strength of up to 1500 Oe. After being reflected from the magnetized sample, the light had changed its linear polarization state for $\pi / 4+\phi_{\text {Kerr }}($ at $t=T / 4)$, or for $3 \pi / 4+\phi_{\text {Kerr }}($ at $t=3 T / 4)$. In order 
to measure the Kerr effect, the reflected light was directed through an analyzer before detection. By changing the orientation of the sample via the goniometer, the intensities of the $\mathbf{M}$ vector were measured in the different direction ( $\phi_{\text {Kerr }}$ has a linear dependence of the $\mathrm{M} !$ !).

As an example two hysteresis loops measured at $\varphi=30^{\circ}$ and $90^{\circ}$ of a $70 \mathrm{~nm}$ thick iron film deposited on Si wafer and irradiated with $350 \mathrm{Mev}$ Au ions to a fluence of $12.5 \times 10^{14} \mathrm{~cm}^{-2}$ are shown in Fig 3.4.3. The important parameters, i.e. the coercive field $\mathrm{H}_{\mathrm{c}}$, residual magnetization $\mathrm{M}_{\mathrm{r}}$ and saturation magnetization $\mathrm{M}_{\mathrm{s}}$ are also shown in this figure.

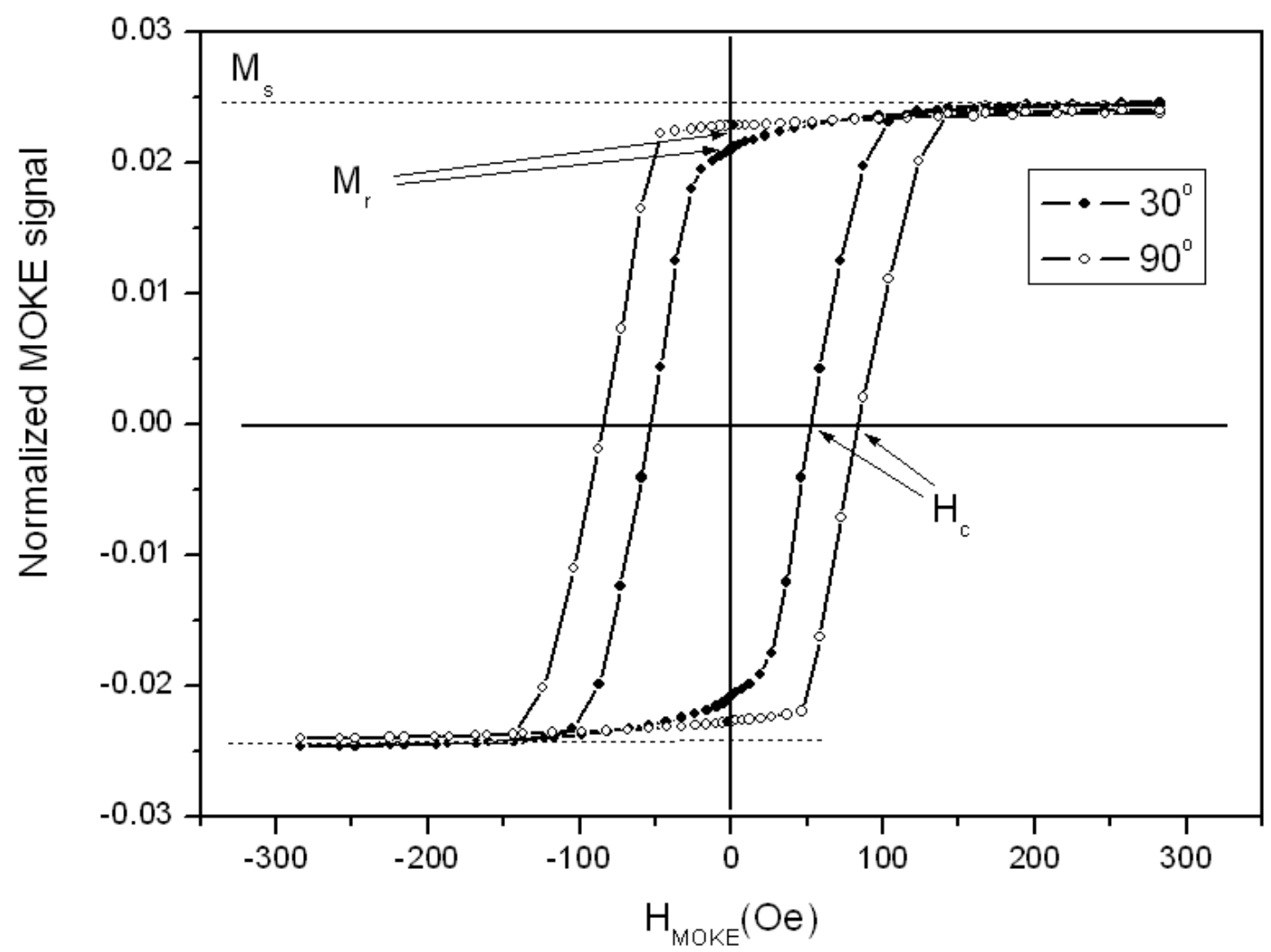

Figure 3.4.3 Hysteresis curves of the $70 \mathrm{~nm}$ Fe/Si sample irradiated with $350 \mathrm{Mev}$ gold ions at a fluence of $12.5 \times 10^{14} \mathrm{~cm}^{-2}$. The coercive field $H_{c}$, residual magnetization $\mathrm{M}_{\mathrm{r}}$, and saturation magnetization $\mathrm{M}_{\mathrm{s}}$ are also shown.

Figure 3.4.5 shows the polar plots of the quantities $H_{c}$ and $M_{r} / M_{s}$ obtained from the MOKE measurements of the same sample. 

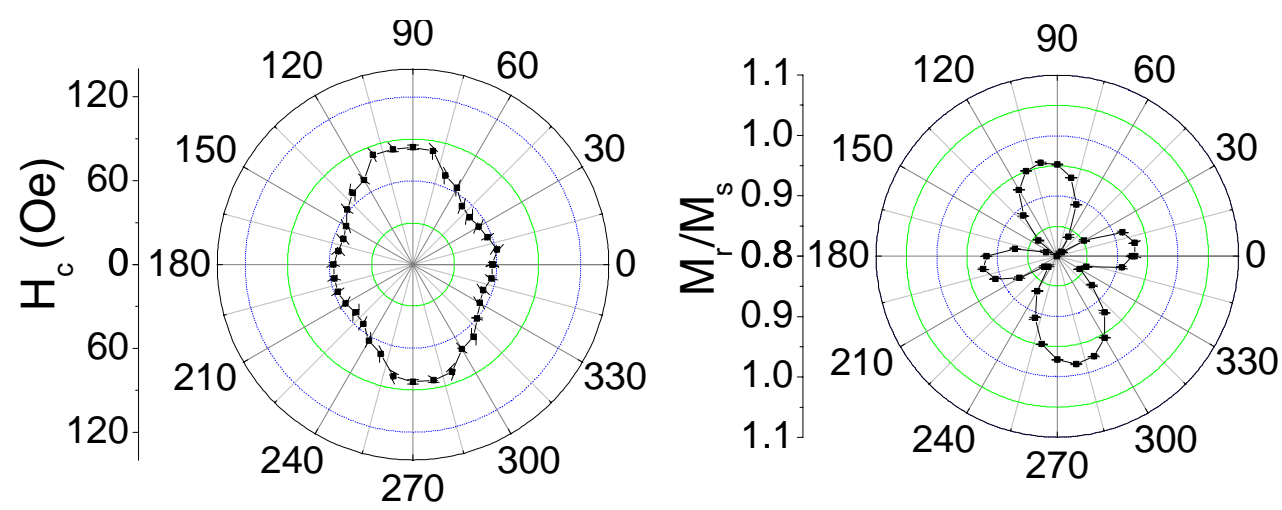

Figure 3.4.5 Polar plots of $\mathrm{H}_{\mathrm{c}}$ and $\mathrm{M}_{\mathrm{r}} / \mathrm{M}_{\mathrm{s}}$ obtained from the MOKE measurements of the $70 \mathrm{nmFe} / \mathrm{Si}$ sample irradiated at a fluence $12.5 \cdot 10^{14} \mathrm{~cm}^{-2}$ with $350 \mathrm{MeV} \mathrm{Au}$ ions.

Visible light only penetrates some $20 \mathrm{~nm}$; therefore, with MOKE only the magnetization in the surface region was probed. On the other hand, the MOMS measurements were performed on the trilayer samples, where the ${ }^{57} \mathrm{Fe}$ layer was inserted between ${ }^{\text {nat }} \mathrm{Fe}$ and the Si substrate. Hence, the biggest contribution to the MOMS spectra came from the ${ }^{57} \mathrm{Fe} / \mathrm{Si}$ interface region, which was mixed during the ion irradiation. Therefore, a comparison of the MOKE and MOMS experiments may lead to different results.

\subsection{X-Ray Diffraction (XRD)}

When monochromatic X-rays hit a crystal with a wavelength comparable to the lattice constant, a constructive interference within the scattered waves may occur. The Bragg's relation gives the condition that must be fulfilled, in order to achieve constructive interference:

$$
\lambda / 2=d \sin \theta \quad(3.4 .1)
$$

where $\lambda$ is the wavelength, $d$ is the spacing between crystallographic planes, and $\theta$ is the incidence angle of the electromagnetic waves relative to the normal of the lattice planes [3-30].

In this work, the $\mathrm{Cu} \mathrm{K}_{\alpha}$ line with a wavelength of $1.54 \mathrm{~nm}$ was used. Bragg-Brentano and grazing incidence geometries were applied. In the Bragg-Brentano geometry, the source of the X-rays and the detector are moving simultaneously, while in the grazing incidence geometry the incoming $X$-ray beam enters at a fixed small angle (up to $5^{0}$ ) relative to the surface of the sample, and the detector is moving. In this way, the X-ray 
spectra provide information on the near-surface part of the sample. The reflections were compared to the powder diffraction database [3-31], and in this way a phase analysis was done.

In Figure 3.5.1, the XRD spectrum of a trilayer sample is displayed, which consists of a $20 \mathrm{~nm}$ thick layer of ${ }^{57}$ Fe deposited on a (111) Si wafer, and a $45 \mathrm{~nm}$ thick layer of natural iron deposited on top of the ${ }^{57}$ Fe layer. In this case, an XRD measurement was performed in glancing incidence geometry. The incidence angle was $3^{\circ}$, and the scanning was done between $42^{\circ}$ and $100.5^{\circ}$, in steps of $0.05^{\circ}$; the measuring time was 60 s/step. The obtained peaks were compared with the PDF JCPDS - ICDD (Powder Diffraction File Joint Committee on Powder Diffraction Standards - International Center for Diffraction Data) database [3-31]. It was found that all peaks match the reflections from the different crystallographic planes of bcc-iron, card number 060696. The peak around $61^{\circ}$ degrees is an artefact.

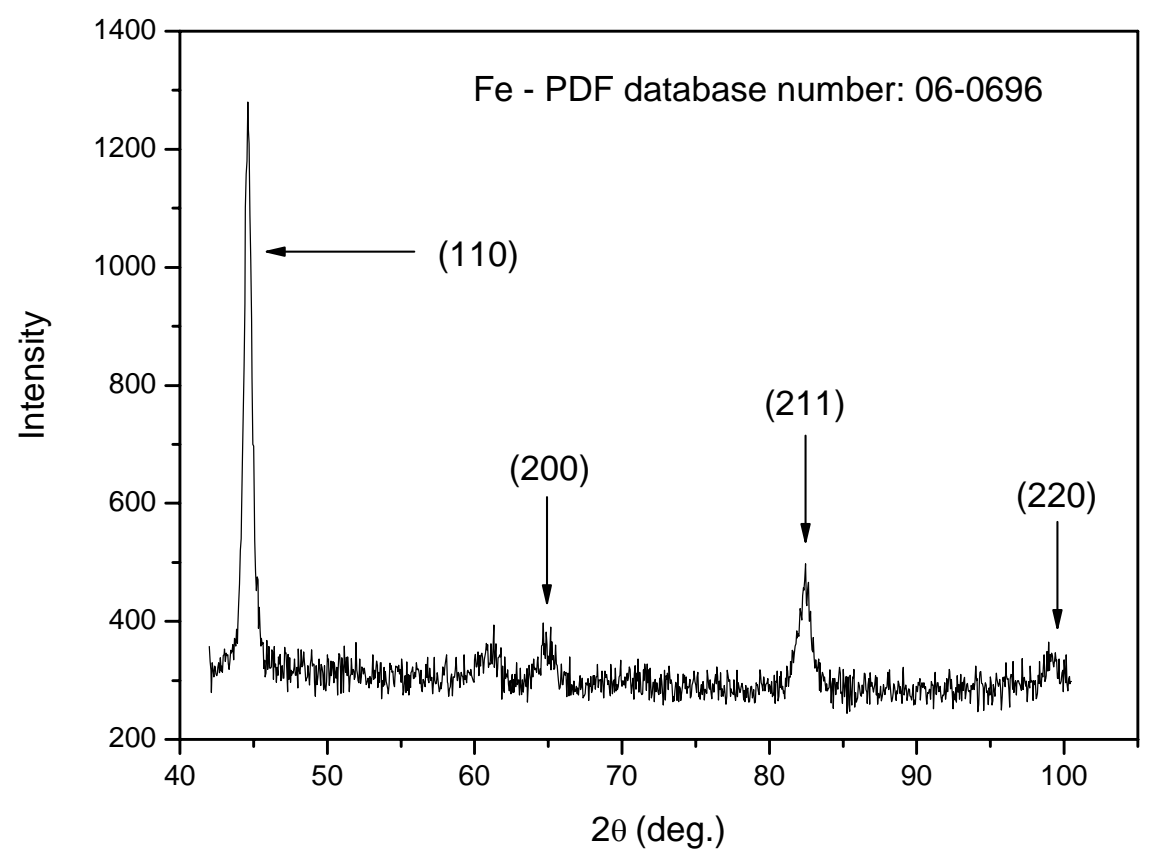

Figure 3.5.1 Glancing incidence XRD spectrum of the sample made of $40 \mathrm{~nm}$ natural $\mathrm{Fe} / 20 \mathrm{~nm}{ }^{57} \mathrm{Fe} / \mathrm{Si}(111)$. 


\section{Nitrogen irradiation and thermal annealing}

The purpose of this part of the work is to investigate the influence of the irradiation of the chemically active nitrogen ions on the ${ }^{57} \mathrm{Fe} / \mathrm{Si}$ bilayer system, as well as the influence of the post-implantation annealing treatment. Ion beam mixing of $\mathrm{Fe} / \mathrm{Si}$ bilayers with noble gas ions had shown the formation of silicide phases and Fe - Si solid solutions [4-1]. In the case of high-fluence nitrogen-ion implantation across the $\mathrm{Fe} / \mathrm{Si}$ interface, one may expect to see some additional iron and silicon nitride phases. Due to the important role of the Fe-N system in industry [4-2,3], its phase diagram has been under investigation for more than a century and is shown in Figure 4.1 [4-4,5].

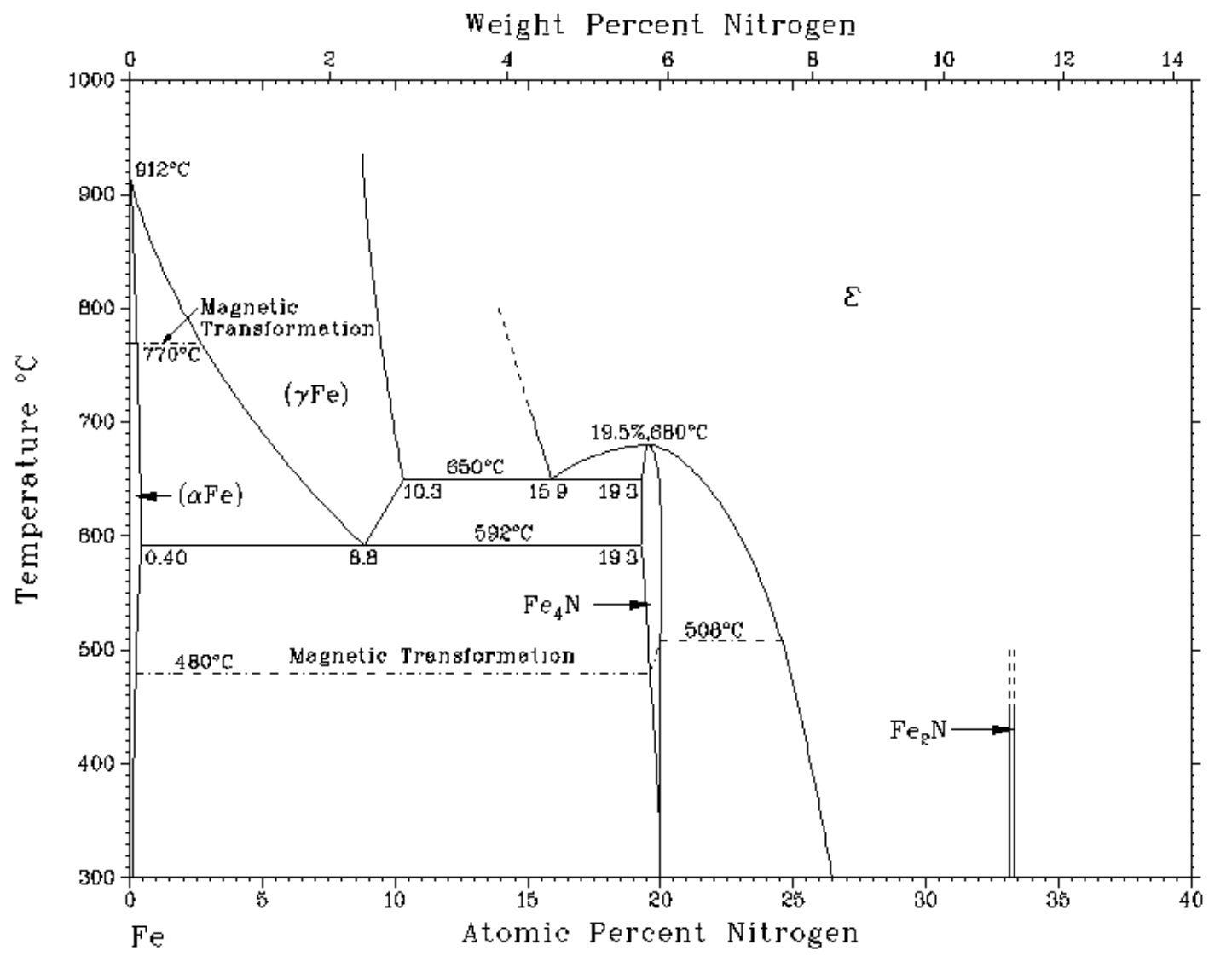

Figure 4.1 Fe - N phase diagram.

Up to about $600{ }^{\circ} \mathrm{C}$, Fe-N system shows three solid solutions: $\alpha, \gamma\left(\mathrm{Fe}_{4} \mathrm{~N}\right), \varepsilon$; a chemical compound $\left(\mathrm{Fe}_{2} \mathrm{~N}\right)$ and two metastabile phases $\left(\alpha^{\prime}\right.$-martensite and $\left.\alpha^{\prime \prime}-\mathrm{Fe}_{16} \mathrm{~N}\right)$. There are numerous comprehensive and well-documented Mössbauer studies about this system [4-6]. 


\subsection{Sample preparation and ${ }^{14} \mathrm{~N}^{++}$ion irradiation}

In this experiment $95 \%$ isotopically enriched ${ }^{57} \mathrm{Fe}$ - films were used. The ${ }^{57} \mathrm{Fe} / \mathrm{Si}$ bilayers were prepared by the PLD method on Si (100) wafers. Before mounting the substrates in the deposition chamber, a standard cleaning procedure was performed, which is described in Chapter 3.1. The thin ${ }^{57} \mathrm{Fe}$ films were deposited at a rate of 0.5 $\mathrm{nm} / \mathrm{s}$ at ambient temperature to a total thickness of $30 \mathrm{~nm}$; the chamber pressure was below $1 \times 10^{-9}$ mbar. A Kr-F excimer laser was used for the evaporation of the material [4-7].

The samples were irradiated to fluences of $0.6,1.0$ and $2.0 \times 10^{17}$ ions $/ \mathrm{cm}^{2}$ with $22 \mathrm{keV}$ ${ }^{14} \mathrm{~N}^{++}$-ions provided by the TESLA facility of the Vinča Institute [4-8]. The pressure in the implantation chamber and in the beam lines was better than $10^{-6} \mathrm{mbar}$. The ion current was in the range of few hundreds of nA. In order to provide a homogeneously irradiated surface, an electrostatic beam sweep-system was used.

\subsection{Results and discussion}

\subsubsection{Phase formation}

An RBS analysis was performed in order to obtain information about the film thickness, possible sputtering effects during nitrogen irradiation, and mixing at the $\mathrm{Fe} / \mathrm{Si}$ interface. Figure 4.2.1 shows the RBS spectra taken from an as-deposited and irradiated ${ }^{57} \mathrm{Fe} / \mathrm{Si}$ sample, as well as the deduced depth profiles. Comparing the integrated counts of the Fe peaks in both samples, the experimental sputtering yield was found to be less than 0.2 atoms/ion. At the highest fluence of $2 \times 10^{17} \mathrm{~N}$-ions / $\mathrm{cm}^{2}$ only $5 \mathrm{~nm}$ would be sputtered off. The decrease in the height of the iron signal, with increasing nitrogen fluence, indicates significant changes in the samples. This effect is most pronounced at the highest fluence and might indicate nitride and/or silicide formation. Both the Fe and Si depth profiles show very similar results. Ion beam mixing will be discussed in Section 4.2.2. 

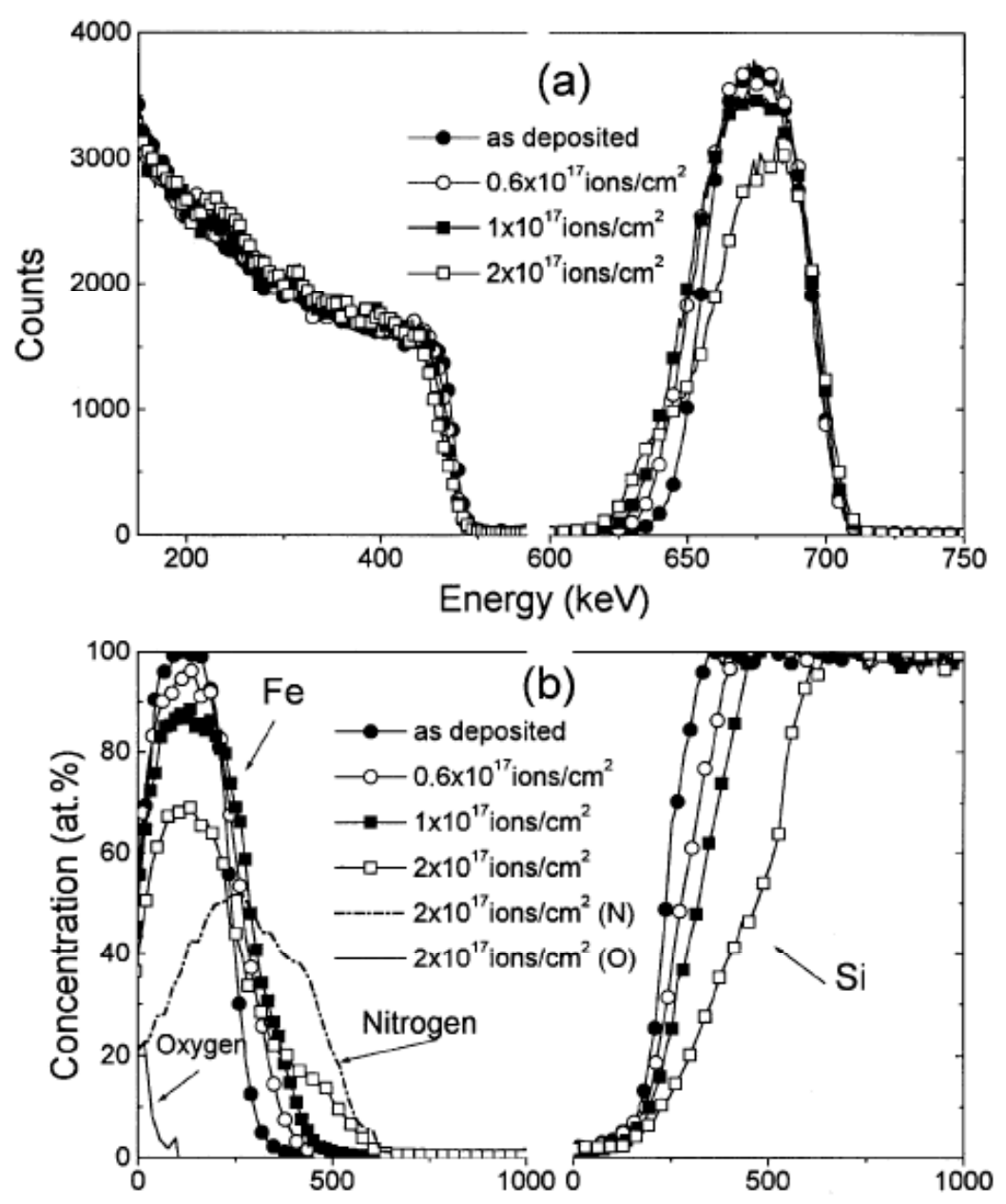

Figure 4.2.1 RBS spectra of the as-deposited and nitrogen (22 keV) irradiated samples to fluences of $0.6,1.0$ and $2.0 \times 10^{17}$ ions $/ \mathrm{cm}^{2}$ (a), as well as their corresponding calculated depth profiles (b).

As RBS was not able to provide clear information about possible nitride or silicide formation, further information about the irradiated samples was obtained by using $\mathrm{XRD}$ and CEM analyses.

Figure 4.2.2 shows X-ray diffraction measurements, performed at a glancing incidence angle of $5^{0}$. As can be seen from the figure, only a few broad, peaks are recorded. A shifting and broadening of the line at an angle of $44^{\circ}$ with an increasing nitrogen fluence indicates the presence of the $\varepsilon-\mathrm{Fe}_{2+} \mathrm{N}$ phase. This diffraction peak is broad, which suggests that the newly formed phase has small crystalline grains. At the highest fluence of $2.0 \times 10^{17}$ ions $/ \mathrm{cm}^{2}$, the well defined $\varepsilon-\mathrm{Fe}_{2} \mathrm{~N}$ phase was achieved. In all the cases a broad peak that corresponds to the (110) Fe reflection is present. This is a clear indication that the formation of the nitride phases was not complete. No other peaks, e.g. from crystalline iron silicides, were observed. 


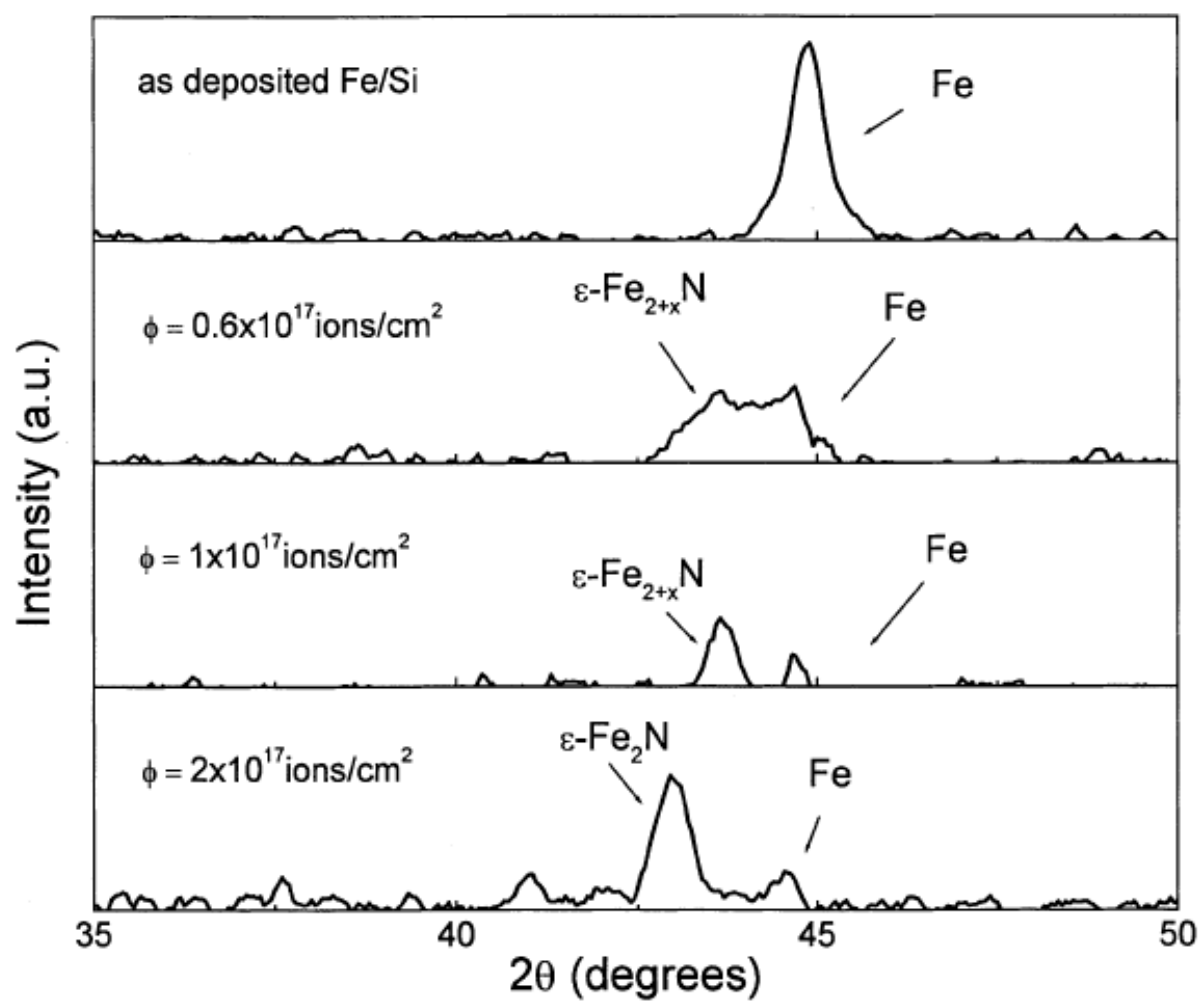

Figure 4.2.2 XRD spectra of the ${ }^{57} \mathrm{Fe} / \mathrm{Si}$ bilayers, before and after irradiation with $22 \mathrm{keV}$ nitrogen ions to various fluences.

Further information about the implanted Fe/Si bilayers was obtained by CEMS measurements. Figure 4.2.3 shows raw and fitted CEMS spectra for the samples irradiated with increasing nitrogen fluence.

For the lowest nitrogen fluence of $0.6 \times 10^{17}$ ions $/ \mathrm{cm}^{2}$, the CEMS analysis showed the presence of $\alpha-\mathrm{Fe}$, with a hyperfine field of $33.0 \mathrm{~T}$. The relative contribution to the spectrum corresponding to this phase was $32.6 \%$. Also, the nitride $\varepsilon-\mathrm{Fe}_{2+\mathrm{x}} \mathrm{N}$ phase was identified, with a relative abundance of 51\%. A nitride paramagnetic phase, which cannot be precisely identified, was also found. These unresolved nitride phases gave a $15.5 \%$ relative contribution to the CEM spectra.

In the sample, which was irradiated to a fluence of $1.0 \times 10^{17}$ ions $/ \mathrm{cm}^{2}$, the CEMS analysis showed one singlet, one doublet and five sextets. The relative abundances of the $\alpha-\mathrm{Fe}$ and $\varepsilon-\mathrm{Fe}_{2+\mathrm{x}} \mathrm{N}$ phases slightly decreased, from $32.6 \%$ to $26 \%$ and from $51 \%$ to $49 \%$, respectively. The origin of a broad sextet, with a hyperfine field of $29.4 \mathrm{~T}$ and an IS of $-0.039 \mathrm{~mm} / \mathrm{s}$ does not provide any clear information about the nitride phase it originates from: is it the $\alpha^{\prime}-\mathrm{FeN}$ or the $\gamma^{\prime}-\mathrm{Fe}_{4} \mathrm{~N}$ phase, or both of them? One additional doublet with a $14 \%$ fraction indicates the possible presence of the unresolved nitride phase. 


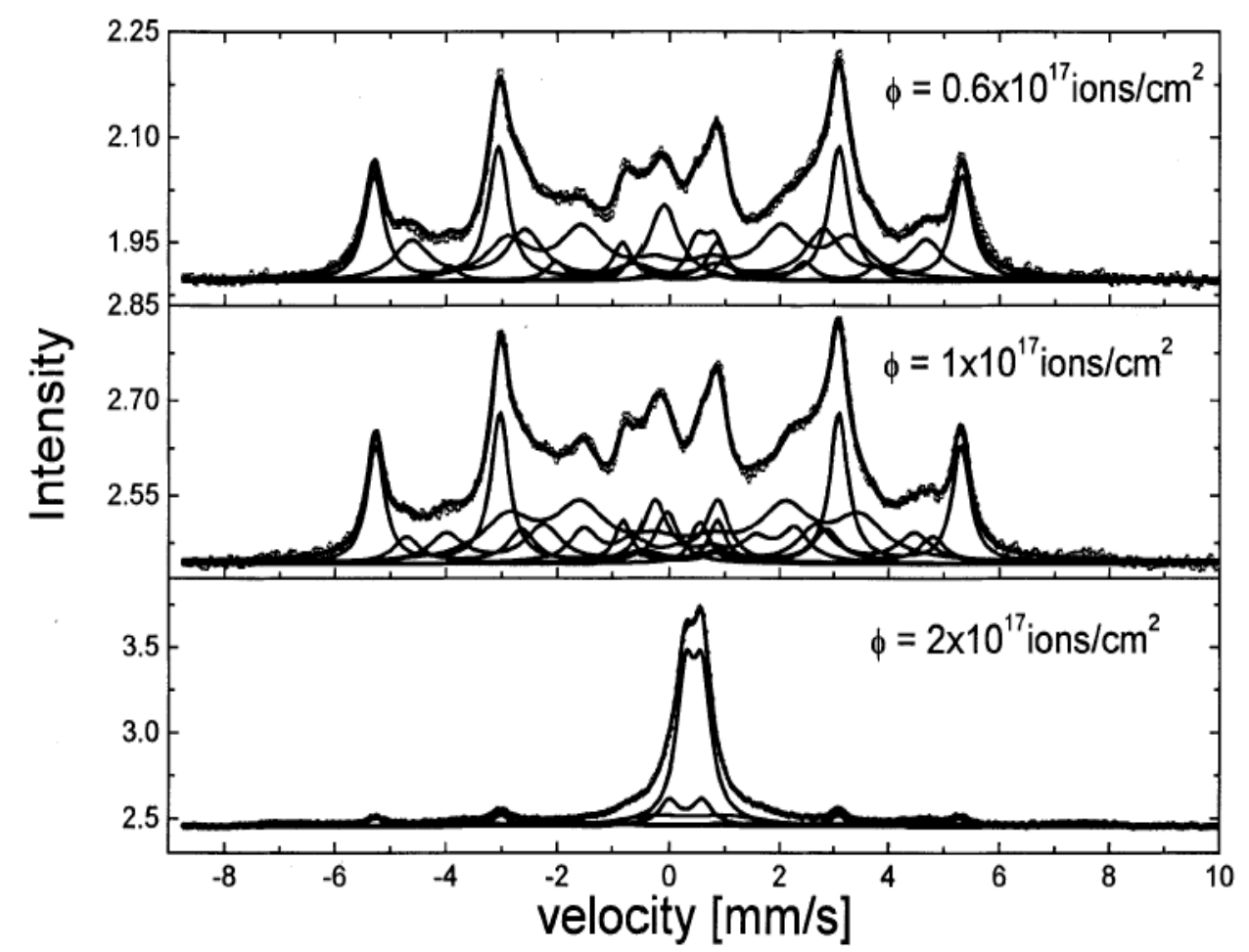

Figure 4.2.3 CEMS measurements of a ${ }^{57} \mathrm{Fe} / \mathrm{Si}$ bilayers irradiated with 22 $\mathrm{keV} \mathrm{N} \mathrm{N}^{++}$ions to $0.6 \times 10^{17}, 1.0 \times 10^{17}$ and $2.0 \times 10^{17} \mathrm{ions} / \mathrm{cm}^{2}$.

Finally, the CEM spectra of the sample irradiated at the highest fluence of 2.0x10 17 ions $/ \mathrm{cm}^{2}$ was successfully fitted with two doublets and four sextets. Two doublets were assigned to the $\varepsilon-\mathrm{Fe}_{2} \mathrm{~N}$ phase, with a relative abundance of $61 \%$. One sextet with a relative abundance of 7.6\%, and a hyperfine field of 33.0 T, was assigned to $\alpha$-Fe. The remaining sextets, with a relative abundance of $19 \%$, were assigned to a mixture of the $\alpha$ '-FeN and $\gamma^{\prime}-\mathrm{Fe}_{4} \mathrm{~N}$ phases.

The evolution of the phase transitions derived from CEMS analyses is summarized in Figure 4.2.4. This figure shows that the fraction of the $\alpha$-Fe phase decreased with increasing nitrogen fluence. A complete transformation of $\alpha$-Fe into nitrides is not achieved, not even for the highest fluence. However, the abundance of this phase (7.6 $\%$ ) is considerably lower than the one obtained when implanting nitrogen into bulk iron (20\%) [4-9]. This significant difference can be attributed to the high concentration of defects in the thin film, which develop during the deposition process. These additional defects contribute to the enhanced reaction of iron and nitrogen and in this way the higher relative fractions of nitrides are achieved. 


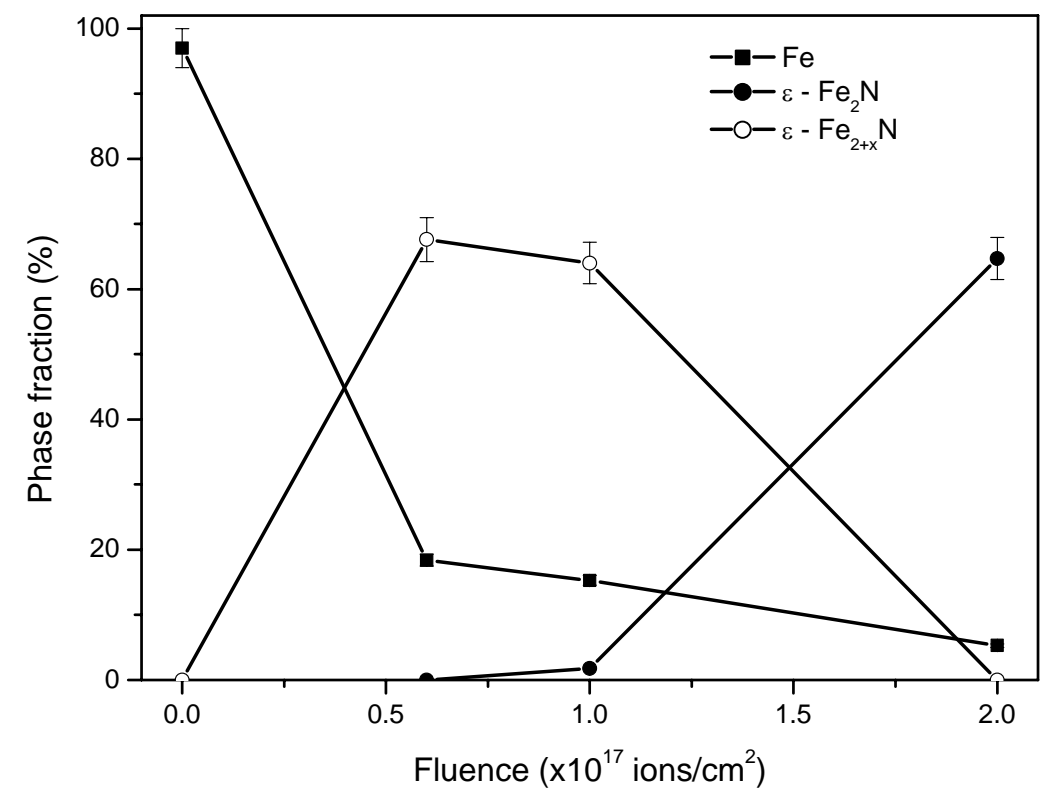

Figure 4.2.4 Nitride phase fractions as observed by CEMS as a function of the ion fluence $\Phi$. The ${ }^{57} \mathrm{Fe}(30 \mathrm{~nm}) / \mathrm{Si}$ samples were irradiated at room temperature with $22 \mathrm{keV}^{14} \mathrm{~N}^{2+}$ ions.

The relative fraction of $\varepsilon-\mathrm{Fe}_{2+\mathrm{x}} \mathrm{N}$ is almost constant after irradiation at low fluences. In the sample irradiated with the highest nitrogen fluence, the nitrogen richer $\varepsilon-\mathrm{Fe}_{2} \mathrm{~N}$ phase appears with a rather large fraction of $61 \%$, while the iron-rich nitride phase $\varepsilon$ $\mathrm{Fe}_{2+\mathrm{x}} \mathrm{N}$ almost disappears. According to CEM measurements, as in the case of XRD measurements, iron silicides are not observed in the samples. In conclusion, highfluence nitrogen irradiation of $\mathrm{Fe}(30 \mathrm{~nm}) / \mathrm{Si}$ bilayers finally produces the $\varepsilon-\mathrm{Fe}_{2} \mathrm{~N}$ nitride, but neither iron-silicide nor silicon nitride [4-10].

\subsubsection{Ion beam mixing}

According to the procedure discussed in Chapter 2, the interface variance $\sigma^{2}$ of the $\mathrm{Fe} / \mathrm{Si}$ mixed region was deduced from the Fe and Si depth profiles as a function of the ion fluence $\Phi$. An average atomic density of Fe and Si of $67.3 \mathrm{at} / \mathrm{nm}^{3}$ was used to convert the depth scale into nm. From the slope of $\Delta \sigma^{2}(\Phi)$ (see Fig 4.2.5) a mixing rate of $\mathrm{k}=\Delta \sigma^{2} / \Phi=0.55(12) \mathrm{nm}^{4}$ was determined.

Neglecting the chemical effects that can be caused by nitrogen ions, the atomic transport across the Fe/Si interface is expected to be dominated by ballistic effects [411,12]. The expression for the ballistic mixing $k_{\text {ball }}$ (Chapter 2.1.3, Eq 2.10) is: 


$$
\Delta \sigma^{2} / \Phi=\mathrm{k}_{\text {ball }}=\frac{1}{3} \Gamma_{0} \xi \frac{\mathrm{R}_{\mathrm{d}}^{2}}{\mathrm{NE}_{\mathrm{d}}} \mathrm{F}_{\mathrm{D}}
$$

where $\Gamma_{0}=0.608, \xi$ is a kinematic factor involving the masses of the colliding target atoms, $\mathrm{N}$ the average atomic density, $\mathrm{R}_{\mathrm{d}} \approx 1 \mathrm{~nm}$ the minimum separation distance for the production of a stable Frenkel pair, $\mathrm{E}_{\mathrm{d}}(\approx 20 \mathrm{eV})$ the average displacement energy, and $F_{D}=0.18 \mathrm{keV} / \mathrm{nm}$ the average energy density deposited at the interface, and it gives $\mathrm{k}_{\text {ball }} \sim 0.03 \mathrm{~nm}^{4}$. It is clear that the experimental mixing rate is at least by one order of magnitude larger than the mixing rate estimated by the ballistic mixing model. This result is in contrast to the experiments performed with Xe ions irradiating strongly bound nitride (AIN or TiN)/metal bilayers [4-13], where the ballistic mixing model proved to be very successful. For Xe ion irradiation of the weakly bound nitride $\mathrm{Ni}_{3} \mathrm{~N}$ on an $\mathrm{Al}$ or $\mathrm{Si}$ substrate, the thermal spike mixing model was successful [4-1,14]. In our case, the absence of any silicide phase excludes thermal spike mixing at the $\mathrm{Fe} / \mathrm{Si}$ interface.

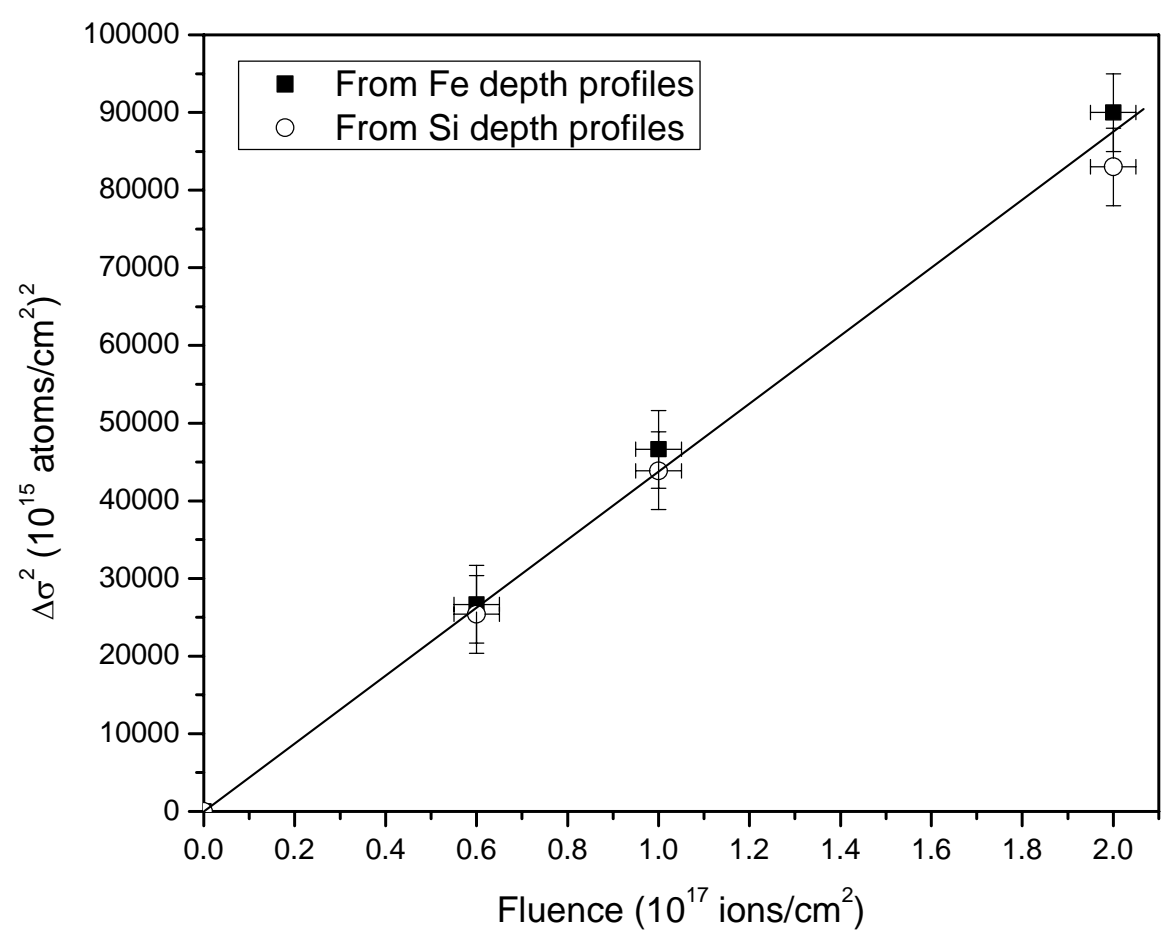

Figure 4.2.5 Interface broadening variance $\Delta \sigma^{2}(\Phi)$ versus ion fluence $\Phi$ in $\mathrm{Fe} / \mathrm{Si}$ bilayers irradiated with $22 \mathrm{keV} \mathrm{N}^{++}$. 


\subsection{Annealing: results and discussion}

The sample irradiated at a fluence of $1 \times 10^{17}$ ions $/ \mathrm{cm}^{2}$ was chosen for a post annealing treatment. The sample was annealed in vacuum at a pressure below $2 \times 10^{-6}$ mbar between $100^{\circ} \mathrm{C}$ and $700^{\circ} \mathrm{C}$. Each annealing step took one hour. After each annealing process, RBS, XRD, and CEMS measurements were performed [4-15]. In Figure 4.3.1, the Si and Fe depth profiles obtained by RBS at various annealing temperatures are shown.

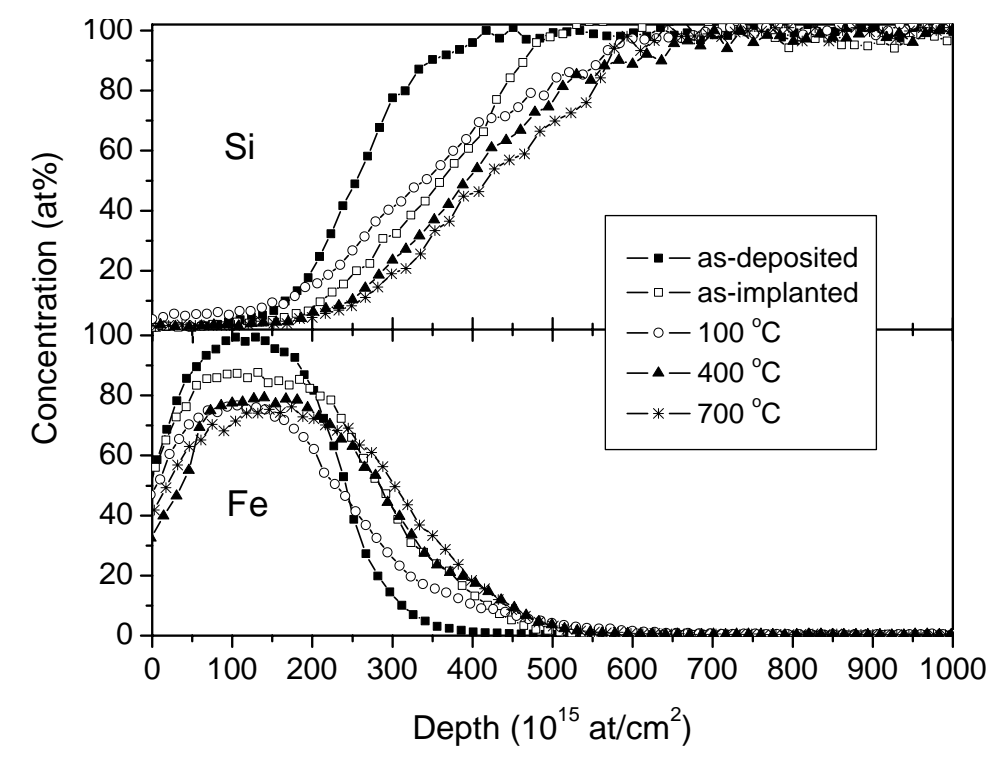

Figure 4.3.1 Selected depth profiles obtained from the RBS spectra taken after post-annealing the sample irradiated to a fluence of $1 \times 10^{17}$ ions $/ \mathrm{cm}^{2}$ for $1 \mathrm{~h}$ at the given temperatures.

After nitrogen irradiation, the Fe concentration decreases from 100 at. \% to about 85 at. \%. As a result of the post - annealing treatment, the maximum Fe concentration decreases further to about 75 at. \% and almost stays constant during all subsequent thermal annealing steps (up to $700{ }^{\circ} \mathrm{C}$ ). In contrast to this behavior of the maximum concentration of iron, the broadening of the Fe/Si interface becomes more and more pronounced when increasing the annealing temperature. The overlapping of the Fe and Si depth profiles indicates that Fe and Si migrate towards each other, and possibly form iron-silicides. However, due to the fact that there is no region with constant Fe and Si concentrations, not a single silicide phase was formed.

The influence of the thermal post-annealing treatment, viewed by XRD, is presented in Fig. 4.3.2. All the scans were performed in glancing-incidence geometry with Xray angle fixed to $3^{\circ}$. 


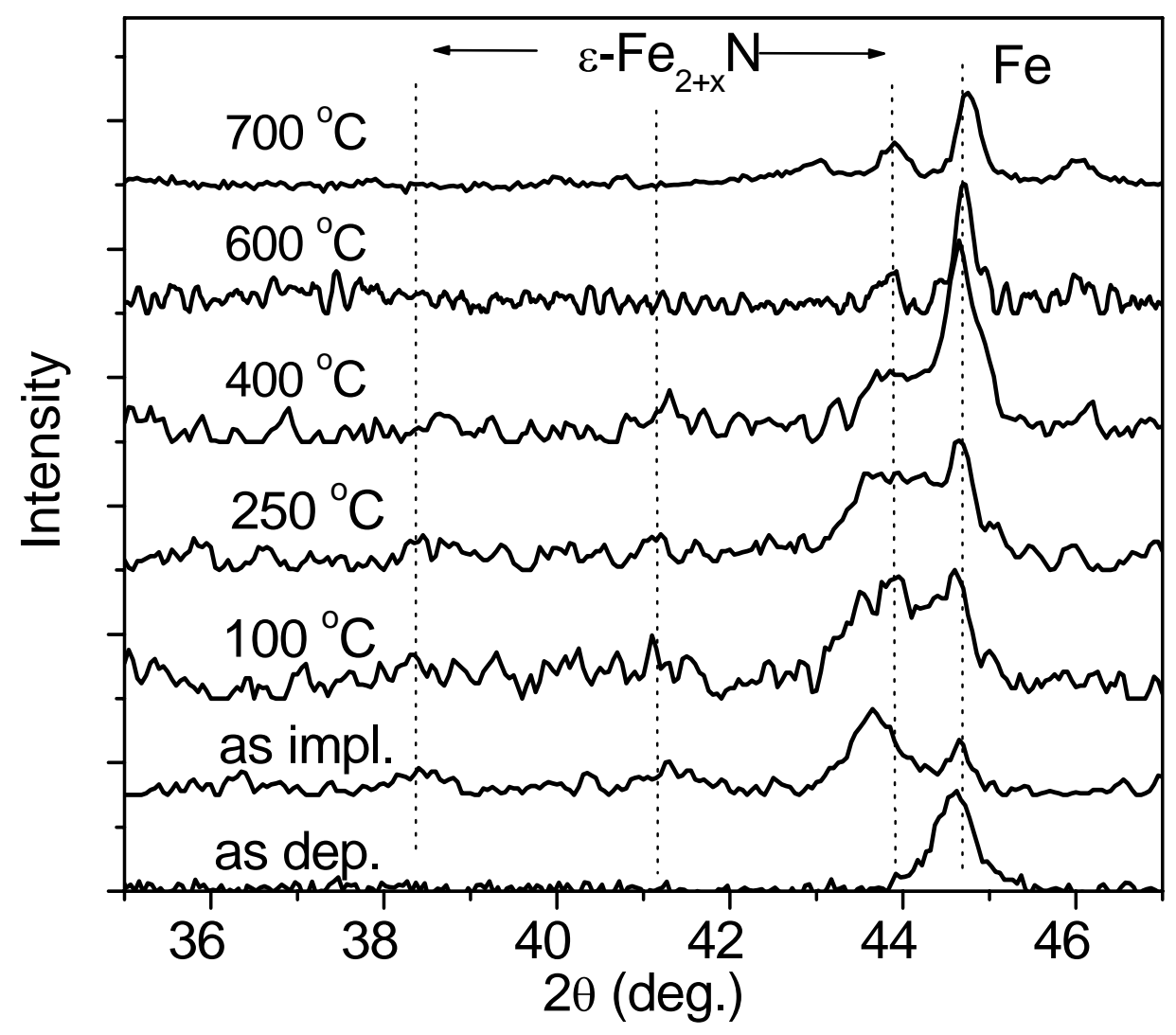

Figure 4.3.2 XRD spectra of the successively annealed sample irradiated to a fluence of $1 \times 10^{17}$ ions $/ \mathrm{cm}^{2}$.

The small thickness of the Fe layer of only $30 \mathrm{~nm}$ did not provide satisfactory counting statistics; consequently, the information obtained from the XRD spectra was insufficient. Besides the peak that corresponds to the iron, we only observed those of $\varepsilon-\mathrm{Fe}_{2+\mathrm{x}} \mathrm{N}$ up to $400{ }^{\circ} \mathrm{C}$. Slight changes in the position of the main peak of this phase suggest a variation of the nitrogen content. 


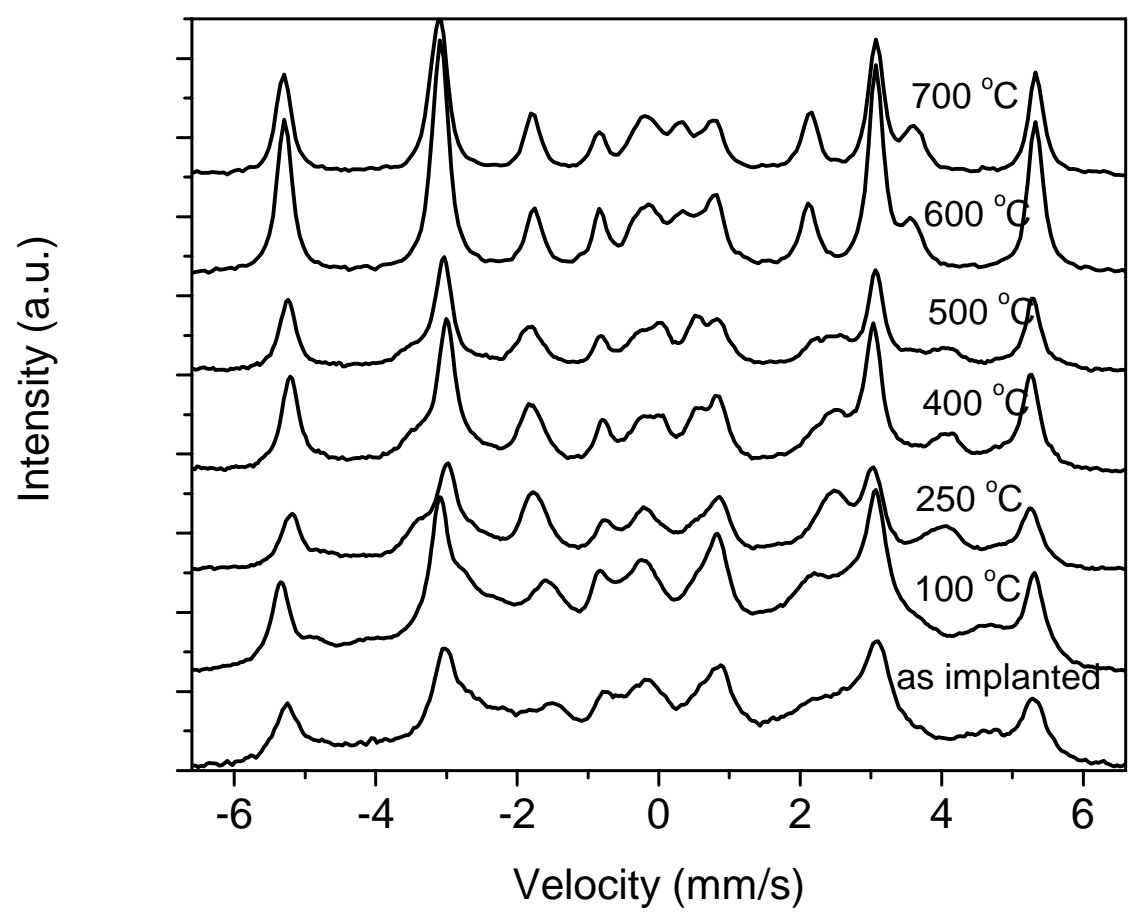

Figure 4.3.3 CEM spectra of the successively annealed ${ }^{57} \mathrm{Fe} / \mathrm{Si}$ bilayer.

The most comprehensive information was gained by the CEMS measurements. Fig. 4.3.3 shows the CEM spectra after each thermal annealing step. In all these cases we identified a sextet with a hyperfine field of $\mathrm{B}_{\mathrm{hf}}=33 \mathrm{~T}$ and a vanishing isomer shift $\delta$ and a quadrupole splitting QS that corresponds to $\alpha$-Fe. We also identified 2-3 additional sextets with fields of $\mathrm{B}_{\mathrm{hf}}=7.0 \mathrm{~T}$ to $29.8 \mathrm{~T}$ corresponding to the $\varepsilon-\mathrm{Fe}_{2+\mathrm{x}} \mathrm{N}$ phase. As the hyperfine field linearly depends on the nitrogen content [4-16], it can also be used to determine the nitrogen concentration in this phase. The three more further sextets with $\mathrm{B}_{\mathrm{hf}}=21.55(4) \mathrm{T}, 21.92(4) \mathrm{T}$ and 34.06(5) $\mathrm{T}$ correspond to the $\gamma^{\prime}$ $\mathrm{Fe}_{4} \mathrm{~N}$ phase [4-17]. Two doublets describe the silicide phase $\beta-\mathrm{FeSi}_{2}$ with isomer shifts of $0.09(1) \mathrm{mm} / \mathrm{s}$ and $0.10(1) \mathrm{mm} / \mathrm{s}$ and quadrupole splittings of $0.54(2) \mathrm{mm} / \mathrm{s}$ and $0.32(1) \mathrm{mm} / \mathrm{s}$, respectively [4-18]. Values of $0.80(\alpha-\mathrm{Fe}), 0.35\left(\varepsilon-\mathrm{Fe}_{3} \mathrm{~N}\right), 0.55\left(\gamma^{\prime}-\right.$ $\left.\mathrm{Fe}_{4} \mathrm{~N}\right)$ and $0.90\left(\beta-\mathrm{FeSi}_{2}\right)$ were used as Debye-Waller factors. One example of the deconvolution of the CEM spectra is shown in Chapter 3. In Fig. 4.3.4, the deduced phase fractions are plotted as a function of the annealing temperature. The predominant phase, up to an annealing temperature of $400^{\circ} \mathrm{C}$, is iron-rich $\varepsilon-\mathrm{Fe}_{2+{ }_{x}} \mathrm{~N}$; its fraction is $75 \%$ at $250^{\circ} \mathrm{C}$. This phase fraction starts to decrease at $400^{\circ} \mathrm{C}$ and is below the detection limit at $600^{\circ} \mathrm{C}$. After annealing at $400^{\circ} \mathrm{C}$, the phase $\gamma^{\prime}-\mathrm{Fe}_{4} \mathrm{~N}$ appears, whose abundance reaches $50 \%$ after the last annealing step at $700^{\circ} \mathrm{C}$. Between $100^{\circ} \mathrm{C}$ 
and $500^{\circ} \mathrm{C}$ we observed a small amount $(\approx 5 \%)$ of amorphous iron-silicide. This fraction may have been underestimated because of its unknown Debye-Waller factor. Only after annealing at $600^{\circ} \mathrm{C}$ and $700^{\circ} \mathrm{C}$ annealing the $\beta-\mathrm{FeSi}_{2}$ iron silicide phase was observed, with a fraction of about $10 \%$.

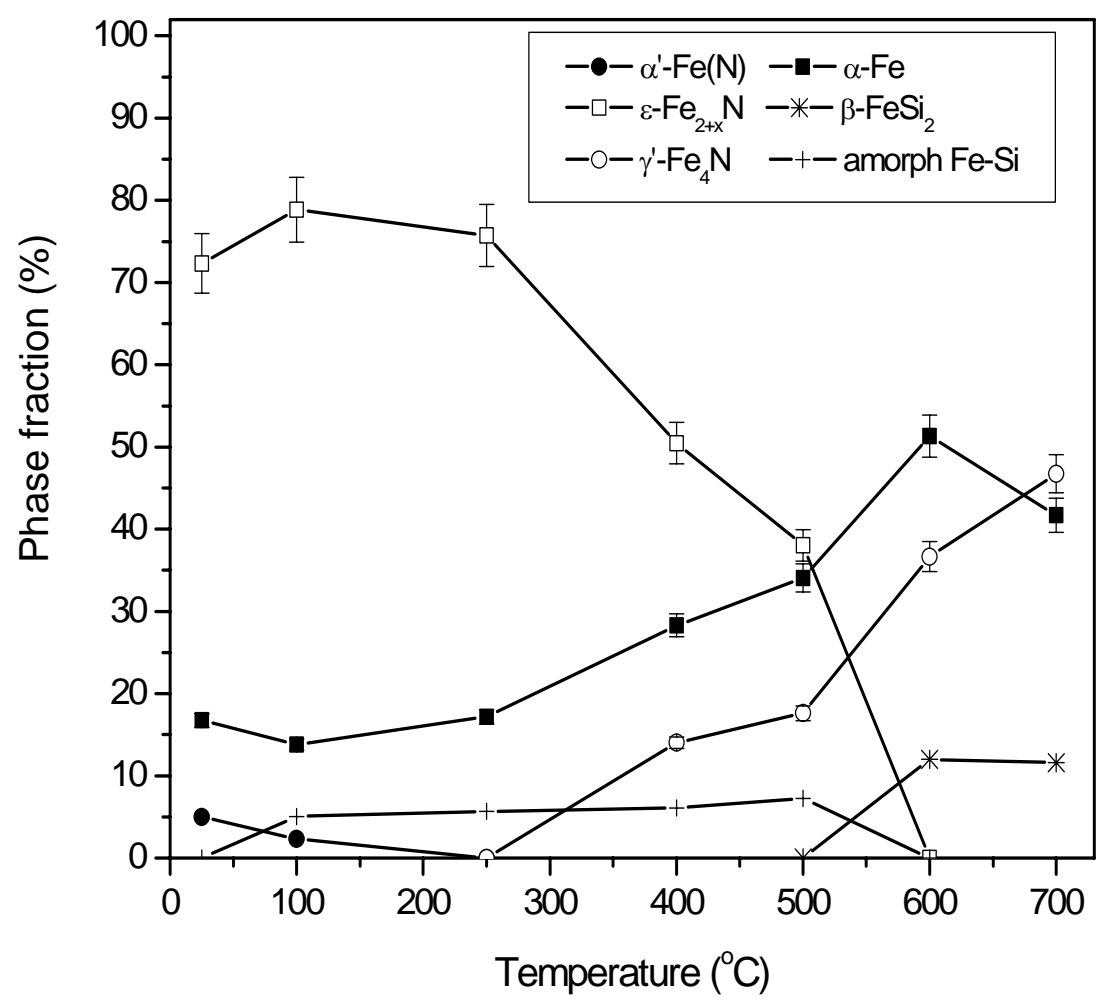

Figure 4.3.4 Phase fractions of various iron-nitrides and iron-silicidies observed by CEMS as a function of the annealing temperature. 
The relative nitrogen content in the sample bound to iron, $\mathrm{C}_{\mathrm{N}-\mathrm{Fe}}$, was calculated from the measured iron nitride phase fractions. As shown in Fig. 4.3.5 this quantity decreases rapidly after the annealing at $250^{\circ} \mathrm{C}$ and continues to decrease up to $600^{\circ} \mathrm{C}$. At this temperature region, the formation of iron-silicides occurs at the Fe/Si interface and at the same time nitride decomposition starts, followed by nitrogen outdiffusion.

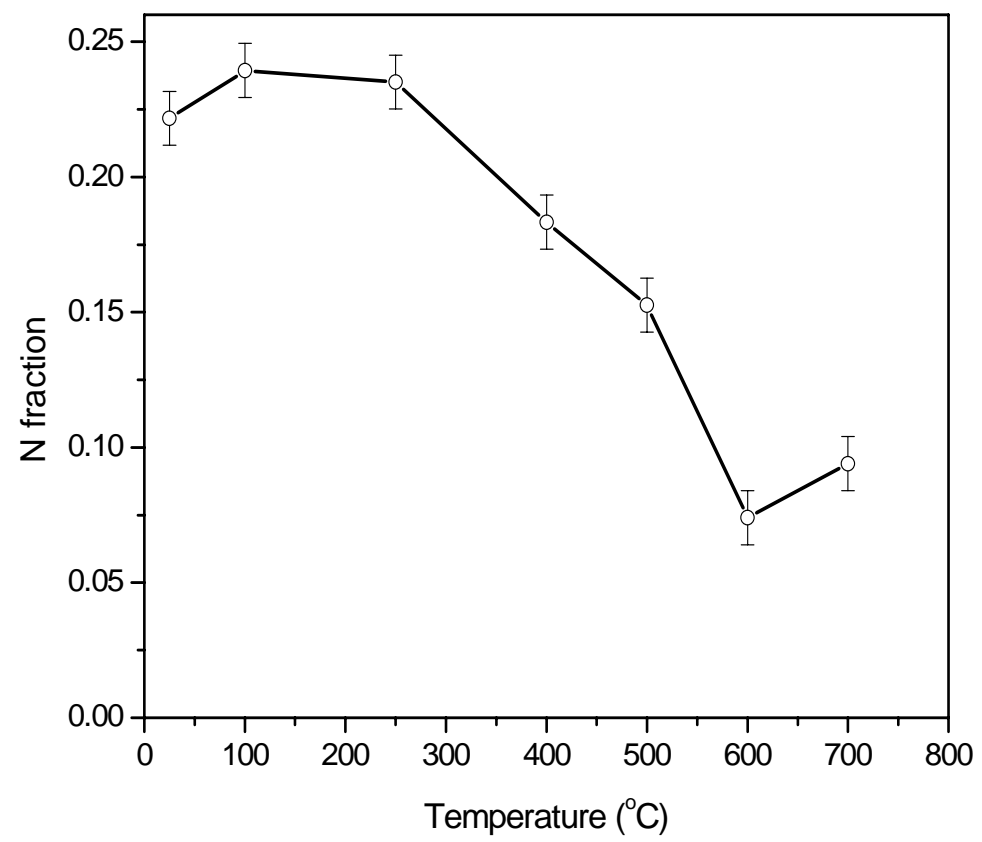

Figure 4.3.5 Total relative nitrogen content bound to iron and observed by CEMS. 


\section{Effects of substrate amorphization and ion charge}

\subsection{Sample preparation}

In these sets of experiments two types of samples were prepared: with and without amorphization of the Si substrate prior to iron deposition. These two different types of bilayers were labeled as Fe/aSi and Fe/Si. Amorphization was performed with a 1.0 $\mathrm{keV} \mathrm{Ar}^{+}$ion beam. The projected range of $1.0 \mathrm{keV} \mathrm{Ar}^{+}$ions and the sputtering yield are 3.5 (16) $\mathrm{nm}$ and 0.8(3) atoms/ion, respectively [5-1]; so all the changes in the $\mathrm{Si}$ substrate are located near the surface of the wafer. Fig. 5.1 shows a scanning electron microscope (SEM) picture of the amorphized sample (aSi), which clearly shows that no additional roughening was induced. Two distinct hillocks are attributed to impurities.

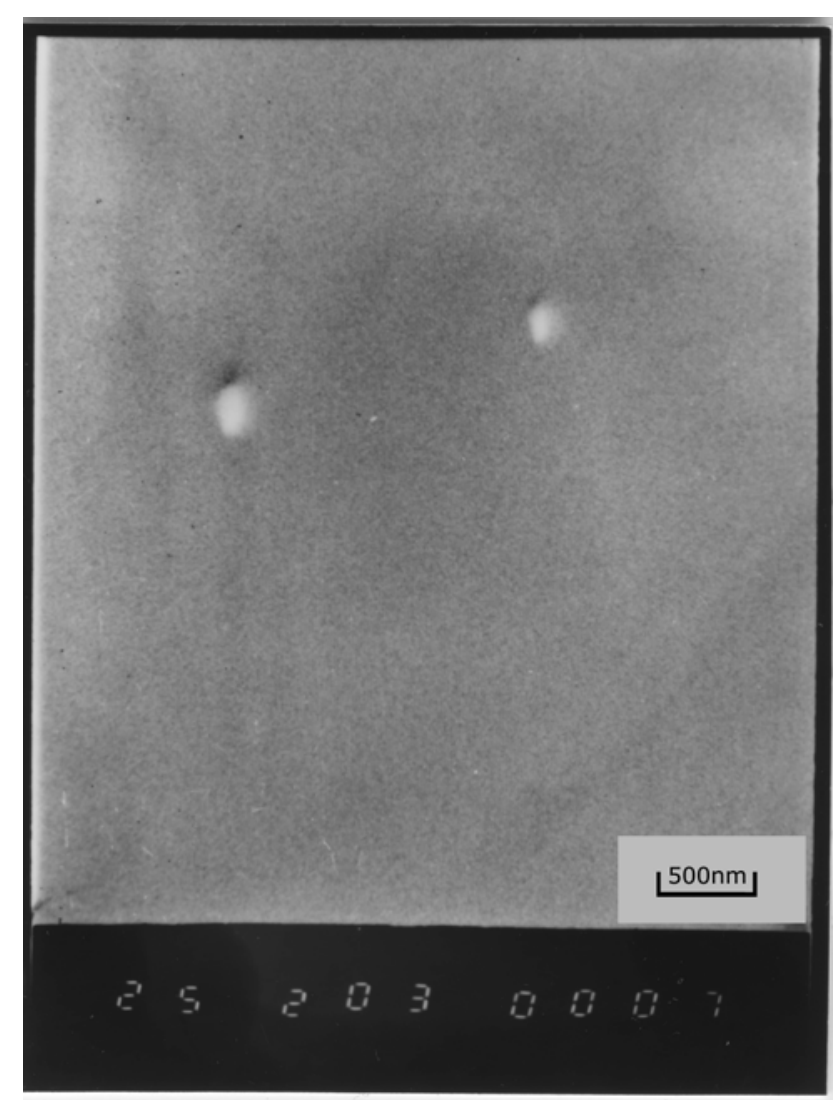

Figure 5.1 SEM picture of the amorphized (aSi) sample: no roughening of the Si surface due to $1.0 \mathrm{keV} \mathrm{Ar}^{+}$beam irradiation. 
Surface roughness can cause significant changes to the values of $\Delta \sigma^{2}$, but this effect was neglected in all these cases as the surface roughness did not change significantly (less than $2 \mathrm{~nm}$ ). From the SEM figure shown in Fig.5.1, any possible roughening of the Si wafer surface due to the $1.0 \mathrm{keV}$ Ar irradiation is well beyond the detection limit.

Iron layers of 30-40 nm thickness were deposited by e-beam evaporation at a rate of $0.5 \mathrm{~nm} / \mathrm{s}$ and a base pressure of $10^{-7} \mathrm{mbar}$ [5-2]. Irradiations of the samples were performed at room temperature with different types of noble gas-ions and different charge states, namely: $\mathrm{Ar}^{1+}$ and $\mathrm{Ar}^{8+}, \mathrm{Kr}^{1+}$ and $\mathrm{Kr}^{11^{+}}, \mathrm{Xe}^{1+}$ and $\mathrm{Xe}^{17+}$. The energies of the ions were chosen in such a way that the deposited energy per ion and unit length $\left(F_{D}\right)$ had its maximum at the iron-silicon interface. Energies of $100 \mathrm{keV}$ were used for argon ions, $180 \mathrm{keV}$ for krypton, and $250 \mathrm{keV}$ for xenon. In this energy range of the ions and thickness of the top iron layer, nuclear energy loss is dominant in the Fe-Si interface region (Chapter 2.1.1, Fig. 2.3). Irradiation experiments with singly-charged ions were performed with the IONAS implanter in Goettingen [5-3], while irradiations with the multiply charged ions were carried out at the TESLA facility at Vinca [5-2]. The experimental mixing rates were obtained by means of RBS, using a $900 \mathrm{keV} \mathrm{He}^{2+}$ beam of the IONAS accelerator; the spectra were analyzed by means of the WiNDF code [5-4].

\subsection{Results and discussion}

Numerous experiments and analyses were done in the scope of this topic. As an example, RBS spectra, the deduced depth profiles and the interface variance $\Delta \sigma^{2}$ versus the fluence $\Phi$ of the $32 \mathrm{~nm}$ thick iron layer on an amorphized Si wafer (aSi), irradiated with $250 \mathrm{keV} \mathrm{Xe}^{1+}$ and different fluences are shown in Figures 5.2 and 5.3. The transformation from "natural RBS depth units" (at/ $\mathrm{cm}^{2}$ ) to "normal depth units" (nm) was performed by using the average atomic density of iron and silicon at the interface: $\rho_{\text {ave }}=67.3$ at. $/ \mathrm{nm}^{3}$. 


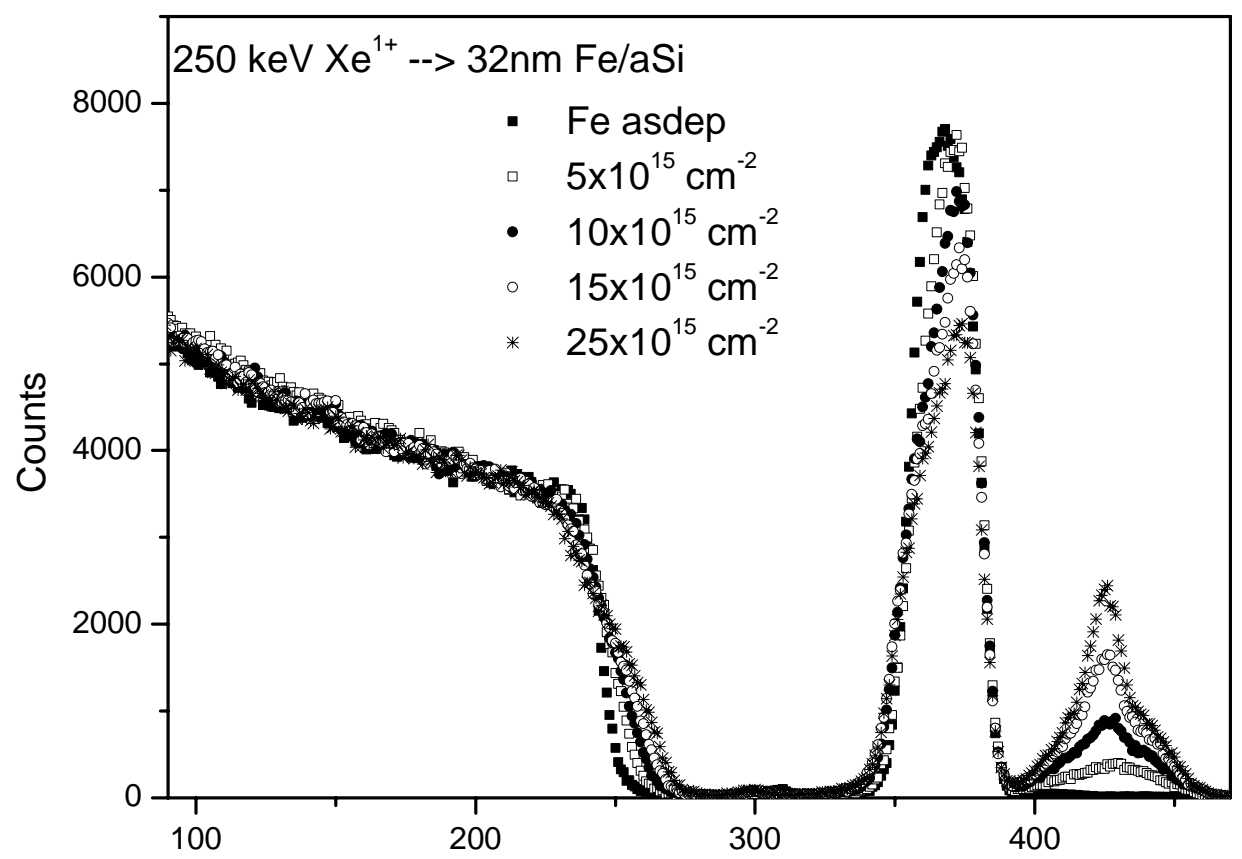

Figure 5.2 Raw RBS spectra of a $32 \mathrm{~nm}$ thick iron layer on an amorphized Si wafer (aSi), irradiated with $250 \mathrm{keV} \mathrm{Xe^{1+ }}$ to a different fluences.
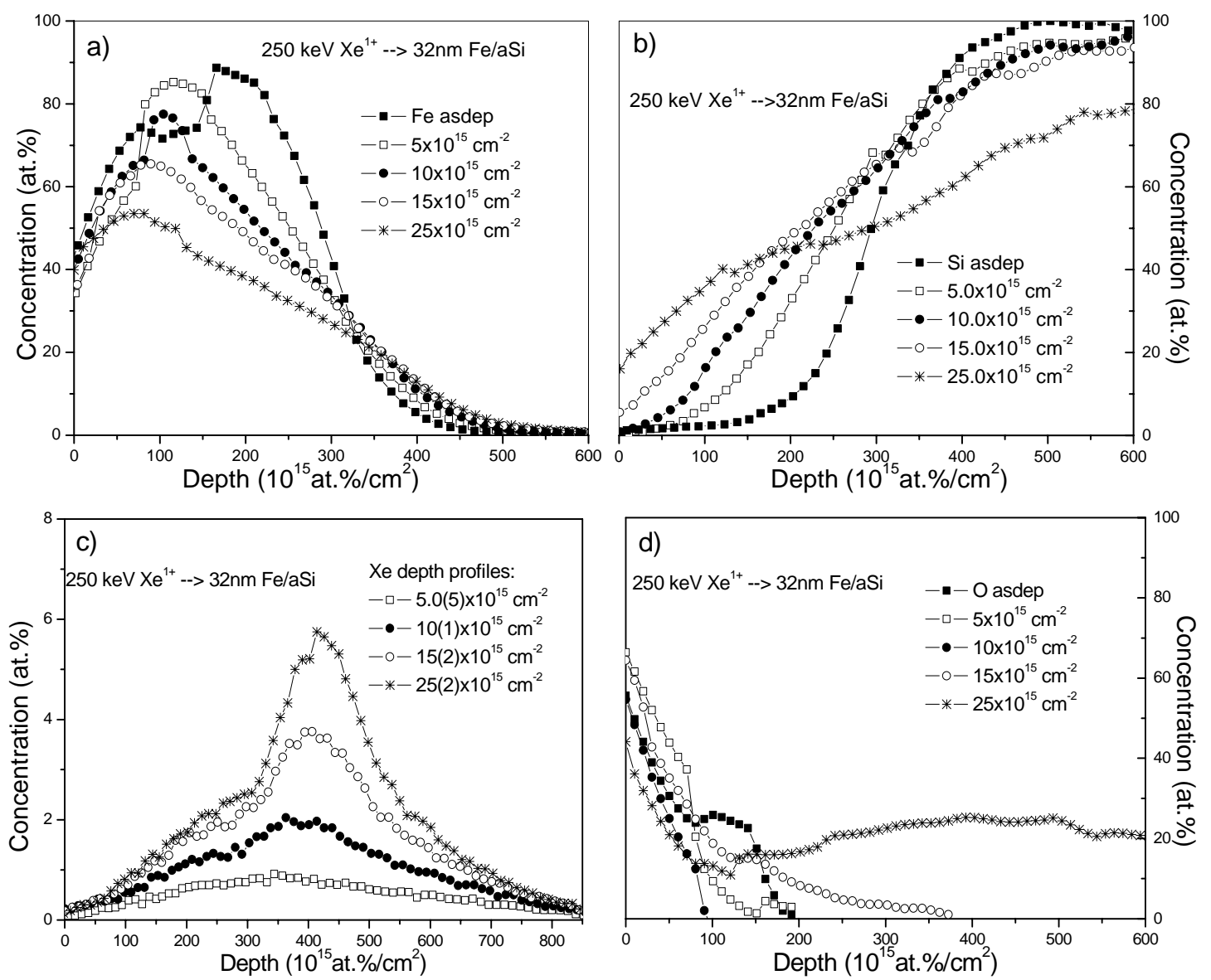

Figure 5.3 Obtained depth profiles of iron (a), silicon (b), xenon (c) and oxygen (d): results of the fitting procedure of the raw RBS spectra shown in Fig 5.2. 
By the integration of the Fe depth profiles (Fig. 5.3a), and a comparison of the irradiated and the as-deposited samples, the experimental sputtering yield was found to be 3.6(6) atoms/ion. Consequently, there was a slight decrease in the iron layer, about $5 \mathrm{~nm}$ for the fluence of $15 \times 10^{15} \mathrm{Xe} / \mathrm{cm}^{2}$. The influence of the sputtering effect can also be noticed at the Si depth profiles: with increasing fluence the silicon edge is shifted toward the top of the sample. Xenon was the heaviest ion used in these sets of experiments, and the sputtering effect was less pronounced in the case of irradiations with argon and krypton ions.

A changes in the variance $\sigma^{2}$ of the silicon depth profiles (Fig. 5.3b), as a function of the ion fluence, provides the slope of the linear curve (Fig 5.4), which is the mixing rate $\Delta \sigma^{2} / \Phi$. The data for the highest fluence were not taken into account in the calculation of the mixing rate. In this case, the Fe-Si interface is heavily contaminated with oxygen during irradiation (see Fig.5.3d) and it represents a different system.

The exact ion fluence was obtained by the integration of the Xe depth profiles (Fig.5.3c)

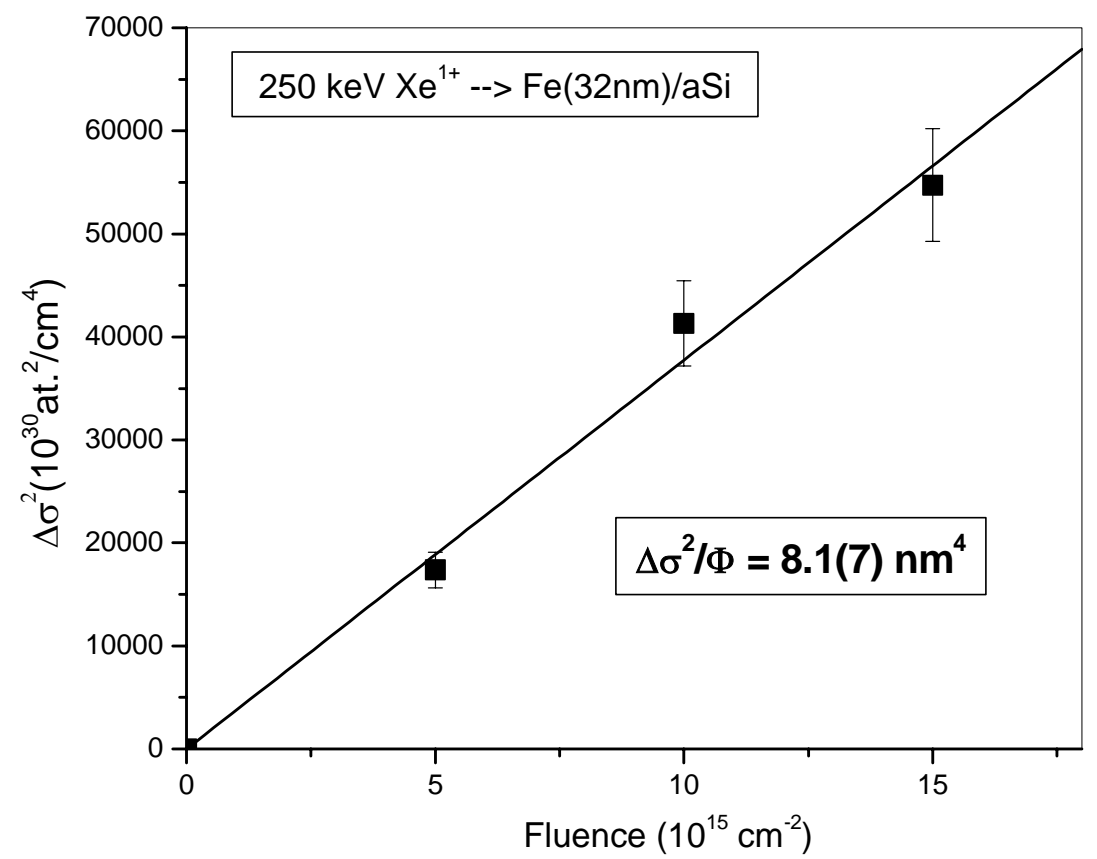

Figure 5.4 Interface variance of the mixed Fe/Si interface $\Delta \sigma^{2}$ as function of the Xe ion fluence $\Phi$. Also slope of the linear curve is indicated $\Delta \sigma^{2} / \Phi$, labeled as mixing rate. 
Fig. 5.5 shows the ion ranges and the deposited energy per ion per unit length, $F_{D}$. These results were obtained using the SRIM code.

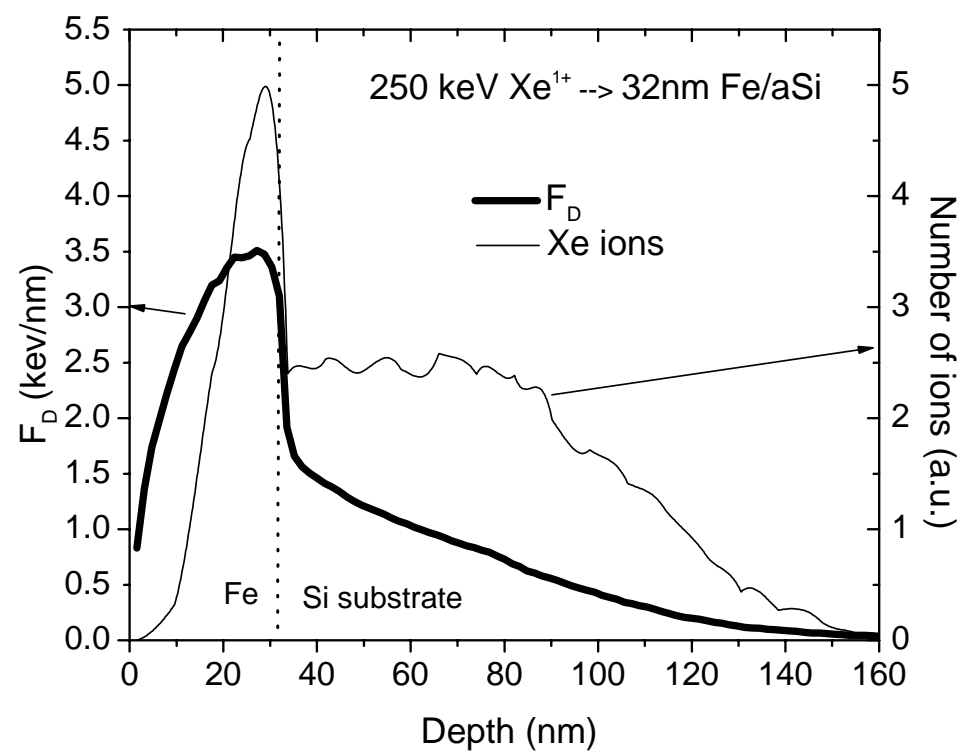

Figure 5.5 Xe implantation distribution and deposited energy per ion per unit length, $\mathrm{F}_{\mathrm{D}}$, calculated by means of the SRIM code.

As can be seen in Fig. 5.5, most Xe ions rested in the Si substrate, but their energy was chosen in such a way that the deposited energy reaches its maximum at the $\mathrm{Fe}$ aSi interface. The deposited energy is slightly changed with increasing ion fluence, due to the sputtering effect; however, this effect was not taken into account.

Equivalent calculations were performed in all these cases, and the deduced mixing rates are summarized in Table 5.1.

Table 5.1 Summary of the irradiation experiments with singly and multiple charged ions of samples with amorphized and non-amorphized Si substrates before iron deposition, as well as comparison with different ion beam mixing models.

\begin{tabular}{|c|c|c|c|c|c|c|c|}
\hline \multirow{3}{*}{$\begin{array}{l}\text { Ion energy } \\
(\mathrm{keV})\end{array}$} & \multirow{3}{*}{ Ion } & \multicolumn{5}{|c|}{$\begin{array}{c}\text { Mixing rate } \Delta \sigma^{2} / \Phi \\
\left(\mathrm{nm}^{4}\right)\end{array}$} & \multirow{3}{*}{$\begin{array}{c}\mathrm{F}_{\mathrm{D}} \\
(\mathrm{keV} / \mathrm{nm})\end{array}$} \\
\hline & & \multicolumn{2}{|c|}{$\begin{array}{l}\text { Experimental, for two } \\
\text { types of substrates }\end{array}$} & \multicolumn{3}{|c|}{ Model } & \\
\hline & & $\mathrm{Fe} / \mathrm{aSi}$ & $\mathrm{Fe} / \mathrm{Si}$ & Ball & Local & Global & \\
\hline \multirow{2}{*}{100} & $\mathrm{Ar}^{1+}$ & $2.7(3)$ & $1.5(2)^{*}$ & \multirow{2}{*}{0.18} & \multirow{2}{*}{1.4} & \multirow{2}{*}{0.5} & \multirow{2}{*}{1.0} \\
\hline & $\operatorname{Ar}^{8+}$ & $3.3(5)$ & $2.1(2)^{*}$ & & & & \\
\hline \multirow{2}{*}{180} & $\mathrm{Kr}^{1+}$ & $4.0(3)$ & $2.6(2)$ & \multirow{2}{*}{0.28} & \multirow{2}{*}{2.9} & \multirow{2}{*}{2.0} & \multirow{2}{*}{2.0} \\
\hline & $\mathrm{Kr}^{11+}$ & $4.3(9)$ & $2.7(5)$ & & & & \\
\hline \multirow{2}{*}{250} & $\mathrm{Xe}^{1+}$ & $8.1(7)$ & $4.8(5)^{*}$ & \multirow{2}{*}{0.5} & \multirow{2}{*}{4.0} & \multirow{2}{*}{3.5} & \multirow{2}{*}{2.8} \\
\hline & $\mathrm{Xe}^{17+}$ & $9.3(6)$ & $4.5(5)$ & & & & \\
\hline
\end{tabular}

-Ref.: [5-5] 
Each of the experimental mixing rates shown in Table 5.1 was obtained by analyzing five RBS spectra for the four different fluences of each ion and for the as-deposited samples. In all the cases $\Delta \sigma^{2}$ varied linearly with the fluence and the well-defined slopes $\Delta \sigma^{2} / \Phi$ were obtained. The fluences were in the range between 1 and $28 \times 10^{15}$ ions $/ \mathrm{cm}^{2}$. The mixing rate values labeled with ${ }^{*}$ were taken from Ref. [5-5].

The experimental results, presented in Table 5.1 are shown in the next Figure 5.6.

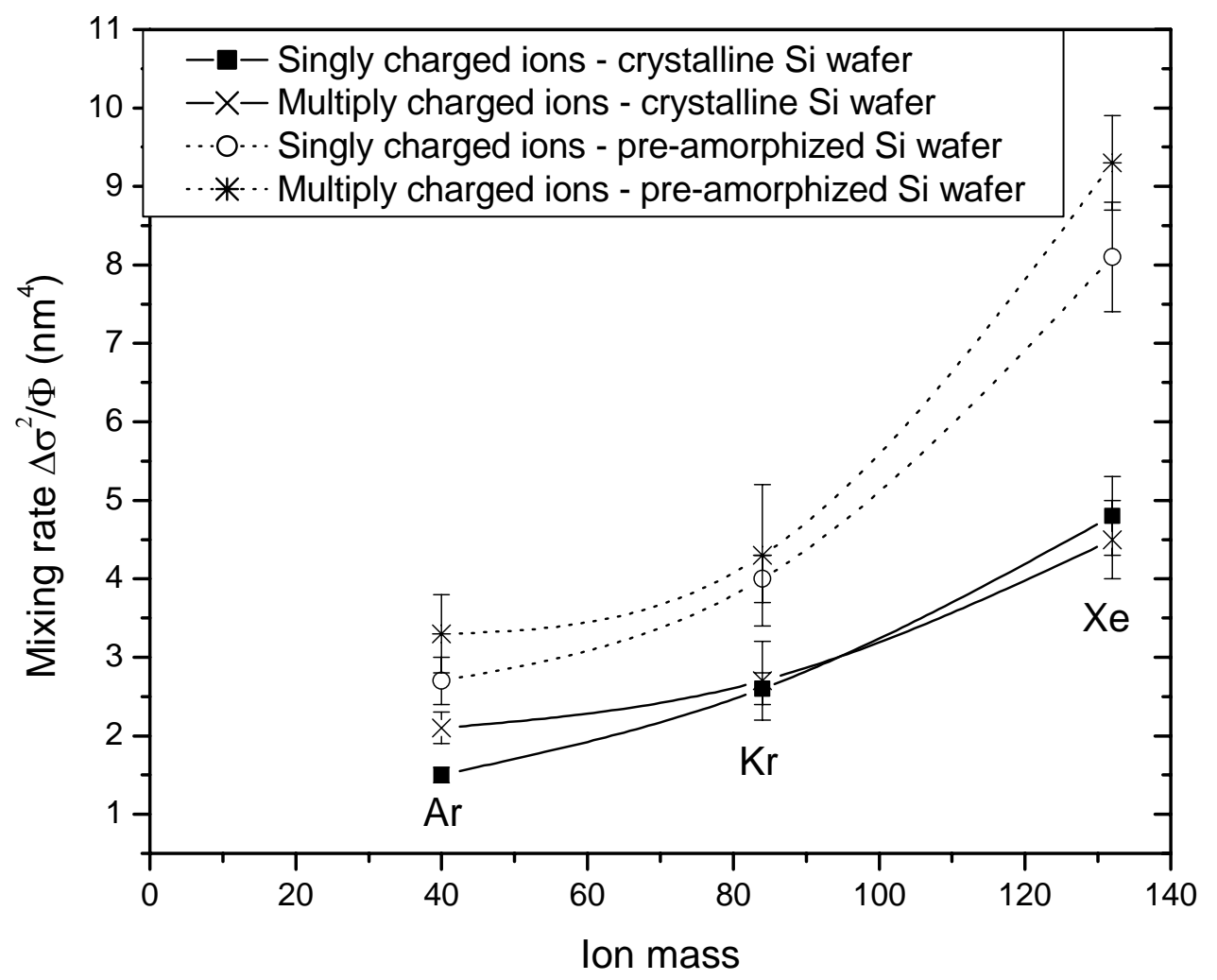

Figure 5.6 Comparison of the mixing rates for singly/multiply charged ions of $\mathrm{Ar}^{+} / \mathrm{Ar}^{8+}, \mathrm{Kr}^{+} / \mathrm{Kr}^{11+}$ and $\mathrm{Xe}^{+} / \mathrm{Xe}^{11^{+}}$on crystalline and pre-amorphized Si wafers before iron deposition.

The most obvious conclusion is that the mixing rate increases with increasing ion mass and deposited energy $F_{D}$. This result was expected and is a well-known effect. However, this large amount of data allows us to draw some more interesting conclusions: 


\section{a) Effects of the ion charge}

To clarify the influence of the ion charge on the mixing rate, an extensive set of experiments was carried out on the Fe/Si bilayers. Irradiations with three different noble-gas ions were performed: Ar, $\mathrm{Kr}$ and $\mathrm{Xe}$; with singly-charge and multiplycharge ion states. For each pair of singly and multiply-charged ion state irradiations, thickness of the top iron layer was the same. Also, both types of Si wafers were used: amorphized and non-amorphized (crystalline). In the case of the $\mathrm{Ar}^{1+} / \mathrm{Ar}^{8+}$ irradiations, the thickness of the iron layer in the Fe/aSi bilayer was $30 \mathrm{~nm}$. For irradiations with $\mathrm{Kr}^{1+} / \mathrm{Kr}^{11+}$ and $\mathrm{Xe}^{1+} / \mathrm{Xe}^{17+}$ the thickness of the top iron layer in $\mathrm{Fe} / \mathrm{aSi}$ and $\mathrm{Fe} / \mathrm{Si}$ bilayer systems was $32 \mathrm{~nm}$.

After a careful calculation of the mixing rates, the values listed in Table 5.1 and also shown in Fig 5.6 were obtained. We found out that, in the chosen range of energies and thicknesses , the ion charge state does not have any influence on the mixing rates. The mixing effect was the same for each ion pair, within error bars.

The investigation of the mixing rate as a function of the ion charge state was triggered by the previously published results in Ref. [5-5]. It was reported that the mixing rate in $\mathrm{Fe} / \mathrm{Si}$ bilayers, due to irradiation with $100 \mathrm{keV} \mathrm{Ar}^{8+}$ ions, is about 50\% larger than the one obtained by irradiation of the same system with $100 \mathrm{keV} \mathrm{Ar}^{1+}$ (Table 5.1). This result was quite surprising, and it is in discrepancy with the mixing rates of $\mathrm{Ta} / \mathrm{Si}$ bilayers irradiated with $250 \mathrm{keV} \mathrm{Xe}^{1+}$ and $\mathrm{Xe}^{19+}$ ions. In this case the mixing rates were the same $\left(\Delta \sigma^{2} / \Phi=3.5(4)\right.$, Ref. [5-5]). It is known that the ions reach their equilibrium charge state after penetrating of 5-7 nm of the conductive solid matter [56], and the thicknesses of the Fe/Si system were in the 30-40 nm range. The sputtering yield in the case of $100 \mathrm{keV}$ Ar ions is negligible, and hence there are no changes in the thickness of the top Fe layer and the deposited energy density. The obviously different behavior of the mixing rates obtained for the $\mathrm{Ar}^{1+} / \mathrm{Ar}^{8+} \rightarrow \mathrm{Fe} / \mathrm{Si}$ and $\mathrm{Xe}^{1+} / \mathrm{Xe}^{19+} \rightarrow \mathrm{Ta} / \mathrm{Si}$ irradiations was explained with the help of the projected range of the ions. In the case of irradiations with argon, 70\% of all ions cross the Fe-Si interface region and are stopped in the Si substrate. In the case of xenon irradiation, only $30 \%$ of the ions manage to cross the Ta-Si interface. It was suggested that the probability that some of the Ar ions with higher charge states are able to cross the interface is larger than in the case of the Xe ions and, consequently, the mixing rates 
are different for $\mathrm{Ar}^{1+}$ and $\mathrm{Ar}^{8+}$ irradiations, and they are the same for $\mathrm{Xe}^{1+}$ and $\mathrm{Xe}^{19+}$ irradiations.

This argumentation was not so convincing. The difference in the charge states of the xenon ions is more than twice as high as the difference between the charge states of the argon ions. If this parameter plays a major role, it would be easier to observe an effect of the charge state in the case of $\mathrm{Xe}^{1+} / \mathrm{Xe}^{19+}$. On the other hand, two different systems were compared: $\mathrm{Fe} / \mathrm{Si}$ and $\mathrm{Ta} / \mathrm{Si}$. This effect was observed only in the $\mathrm{Fe} / \mathrm{Si}$ bilayer. The Fe/Si bilayer system might be especially sensitive to the charge state of ion. Also, the thicknesses of the Fe/Si bilayers were not identical and this may be the reason for the different values of the mixing rates.

The presented results ruled out speculations about the influence of the projected range of the ions and some possible special features of the Fe-Si bilayer system.

b) Effects of the amorphization of the Si substrate prior to the deposition of the iron layer

The obtained mixing rates for this part of the experiment are listed in Table 5.1 and plotted in Fig. 5.6. It is clear that in all the cases preamorphization of the Si substrate leads to higher mixing rates, by a factor of 1.8(2) than those of the crystalline Si substrate. The influence of roughening was not considered because the SEM picture (Fig. 5.1) did not show any significant roughening of the surface of the Si wafer. RBS measurements led to the same result. Figure 5.6 shows the Si depth profiles of the $\mathrm{Fe} / \mathrm{aSi}$ and $\mathrm{Fe} / \mathrm{Si}$ as-deposited samples. RBS is not able to resolve any roughening of the bulk systems, but can supply this information with respect to the interfaces. When alpha particles hit the surface of the bulk material, they will collide with surface atoms with the same energy, regardless of the relative position of the surface atoms to each other. Consequently, the backscattered alpha particles will have the same energy, and the counts will be collected around the same channel, spread by the resolution of the RBS system. When RBS measurements are performed with the bilayer system, due to the different thicknesses of the top layer, introduced by the roughens, the alpha particles will loose different amounts of energy before they hit the substrate layer. Thus the backscattered particles will have slightly different energies, and any spreading of the substrate signal will be larger than for ideal flat surfaces. The spreading of the signal is not only caused by the roughness of the substrate, but also 
by the roughness of the surface of the top layer. In Fig. 5.7 the same slopes of the silicon depth profiles of Fe/aSi and Fe/Si as-deposited bilayers can be seen. The difference between the Si depth profiles of two types of Fe-Si bilayers, pre-amorhized and crystalline Si wafers, is $1 \mathrm{~nm}$, (using the average atomic density of iron and silicon at the interface: $\rho_{\text {ave }}=67.3$ at. $/ \mathrm{nm}^{3}$ ). The spreading is caused only by the resolution of the RBS system used. So, within $1 \mathrm{~nm}$, according to the RBS analysis, interface and surface of the two types of bilayers, Fe/aSi and Fe/Si, can be considered to be flat.

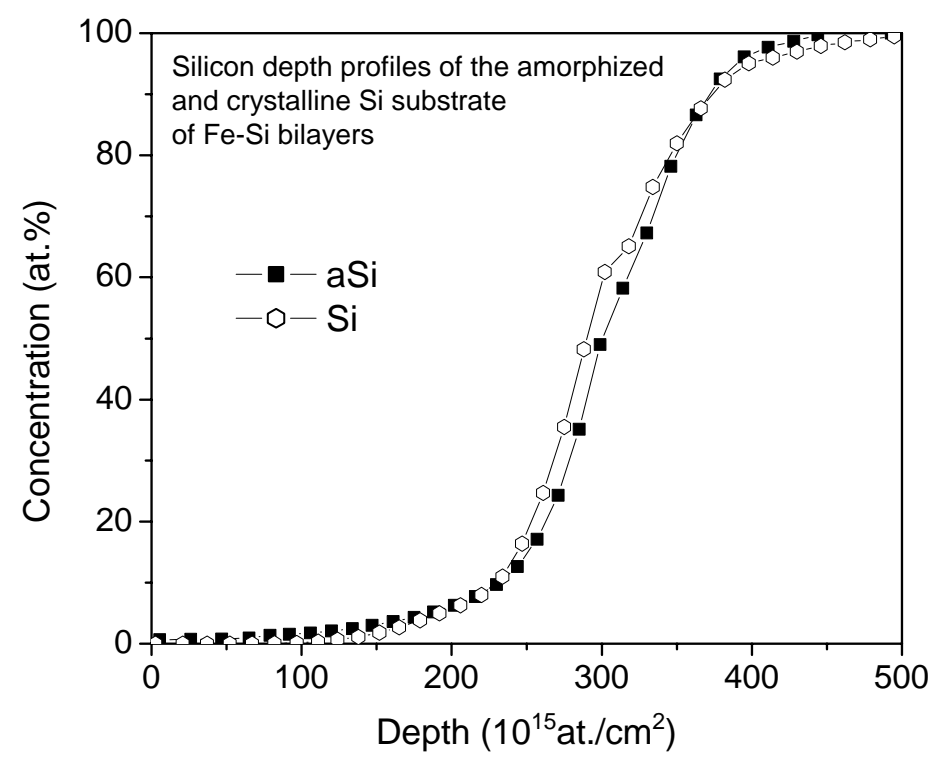

Figure 5.7 Si depth profiles of the Fe/aSi and Fe/Si as-deposited samples.

The only remaining effect of the treatment of the Si substrate with a low-energy $\mathrm{Ar}^{+}$ beam prior to the deposition of an iron layer is amorphization. Argon ions break the crystal lattice of the silicon, thus the Si atoms are more mobile and can easily diffuse in to the iron layer during the irradiation process. Their mobility is almost doubled, which is shown by the larger mixing rate.

\section{c) A comparison of experimental and model mixing rates}

It is obvious (see Table 5.1) that ballistic mixing (Chapter 2.1a) underestimates the experimental mixing rates by one order of magnitude, as expected for heavy-on bilayer-systems. Either local or global spikes can explain the results for the 
experimental mixing rates. The local spike mixing model (Chapter 2.1b) reproduces the mixing rates in the mixing experiments with the Fe/Si system very well. On the other hand, the global spike mixing model (Chapter 2.1c) is less successful in all the cases. This model supplies the best prediction for irradiation experiments with Xe ions. Here the highest value of the deposited energy, $F_{D}$, is $2.8 \mathrm{keV} / \mathrm{nm}$. In our set of experiments, the transition between local and global spikes appears to occur at a deposited energy density higher than $2.8 \mathrm{keV} / \mathrm{nm}$.

For higher mixing rates obtained in the experiments with Fe/aSi bilayers, none of the models is suitable and modifications of the mixing models are necessary. 


\section{Swift ion irradiation with $350 \mathrm{MeV}$ Au ions}

In contrast to the experiments described previously, where the nuclear stopping of the ions played an important role, in this set of experiments nuclear stopping is negligible and electronic stopping is predominant. At those chosen energy of $350 \mathrm{MeV}$ Au ions it is possible to investigate interface mixing and phase formation in the regime of pure electronic stopping.

\subsection{Sample preparation}

Two sets of samples were prepared for the swift heavy ion irradiation experiments: ${ }^{\text {nat }} \mathrm{Fe}(50 \mathrm{~nm}) / \mathrm{Si}$ bilayers and ${ }^{\text {nat }} \mathrm{Fe}(45 \mathrm{~nm}) /{ }^{57} \mathrm{Fe}(20 \mathrm{~nm}) / \mathrm{Si}$ trilayers. The ${ }^{\text {nat }} \mathrm{Fe}(50 \mathrm{~nm}) /$ Si bilayers were prepared in a UHV chamber [6-1] by electronbeam evaporation of the iron. The deposition rate was about $0.5 \mathrm{~nm} / \mathrm{s}$, and the base pressure in the chamber was below $4 \cdot 10^{-8}$ mbar.

The trilayer films of ${ }^{\mathrm{nat}} \mathrm{Fe}(45 \mathrm{~nm}) /{ }^{57} \mathrm{Fe}(20 \mathrm{~nm}) / \mathrm{Si}$ were deposited at room temperature by pulsed laser deposition. The ambient pressure in the deposition chamber was below $10^{-8}$ mbar. These samples, with a ${ }^{57} \mathrm{Fe}$ sandwiched layer, were used for extensive CEMS and MOMS measurements. They were prepared at the Leibniz Institut für Festkörper und Werkstoffforschung (IFW), Dresden.

The samples were homogeneously irradiated across a $7 \times 7 \mathrm{~mm}$ area with $350 \mathrm{MeV}$ $\mathrm{Au}^{26+}$ ions and a $100 \mathrm{nA}$ beam at the ECR-RFQ cyclotron accelerator facility of the Hahn-Meiter-Institut, Berlin [6-2] at fluences of up to $5 \times 10^{15}$ ions $/ \mathrm{cm}^{2}$. The irradiations were performed either at room temperature or at $92 \mathrm{~K}$. In all the cases the ambient pressure during irradiation process was below $10^{-6} \mathrm{mbar}$.

\subsection{Results and discussion}

SRIM simulations [6-3] of the irradiation of ${ }^{\text {nat }} \mathrm{Fe}(45 \mathrm{~nm}) /{ }^{57} \mathrm{Fe}(20 \mathrm{~nm}) / \mathrm{Si}$ trilayers with $350 \mathrm{MeV} \mathrm{Au}^{26+}$ ions suggest that the nuclear stopping power in the iron layers and in the Si substrate near the interface are: $S_{n}(\mathrm{Si})=23 \mathrm{eV} / \mathrm{nm}$ and $\mathrm{S}_{\mathrm{n}}(\mathrm{Fe})=90$ $\mathrm{eV} / \mathrm{nm}$. The electronic stopping is by three orders of magnitude higher and its values 
are: $\mathrm{S}_{\mathrm{e}}(\mathrm{Si})=19 \mathrm{keV} / \mathrm{nm}$ and $\mathrm{S}_{\mathrm{e}}(\mathrm{Fe})=52 \mathrm{keV} / \mathrm{nm}$. The nuclear and electronic stopping powers are almost constant in the upper $200 \mathrm{~nm}$ of the samples (Fig. 6.2.1.). The projected range of the Au ions is deeply in the Si substrate, and it is about $27 \mathrm{~nm}$.

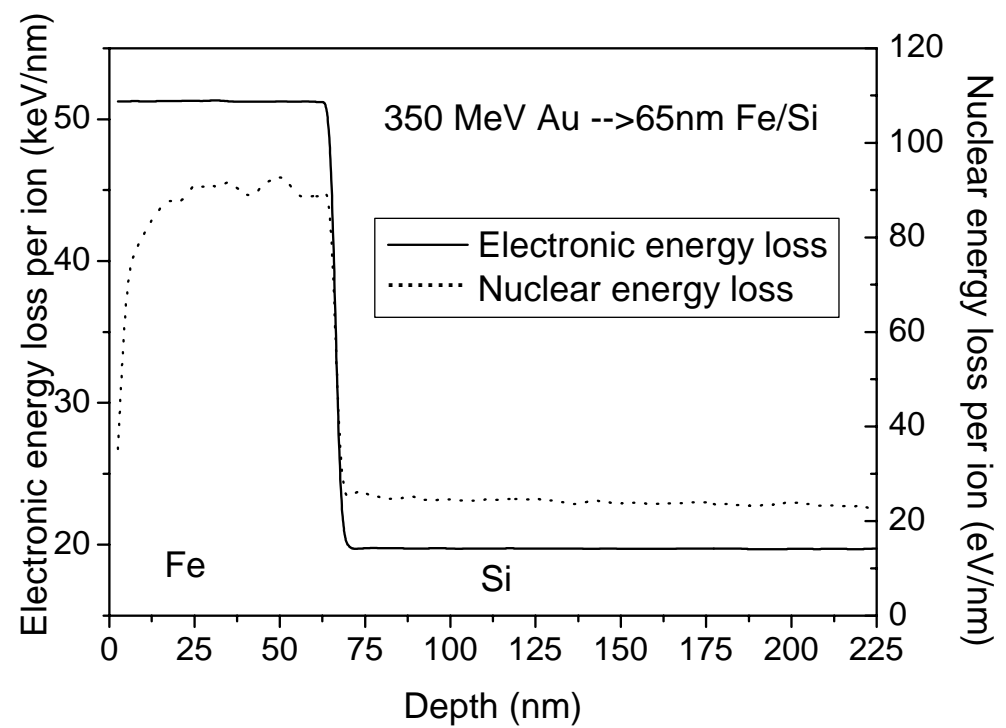

Figure 6.2.1 SRIM simulations of the irradiation of Fe(65 nm)/Si trilayers with $350 \mathrm{MeV}$ Au ions.

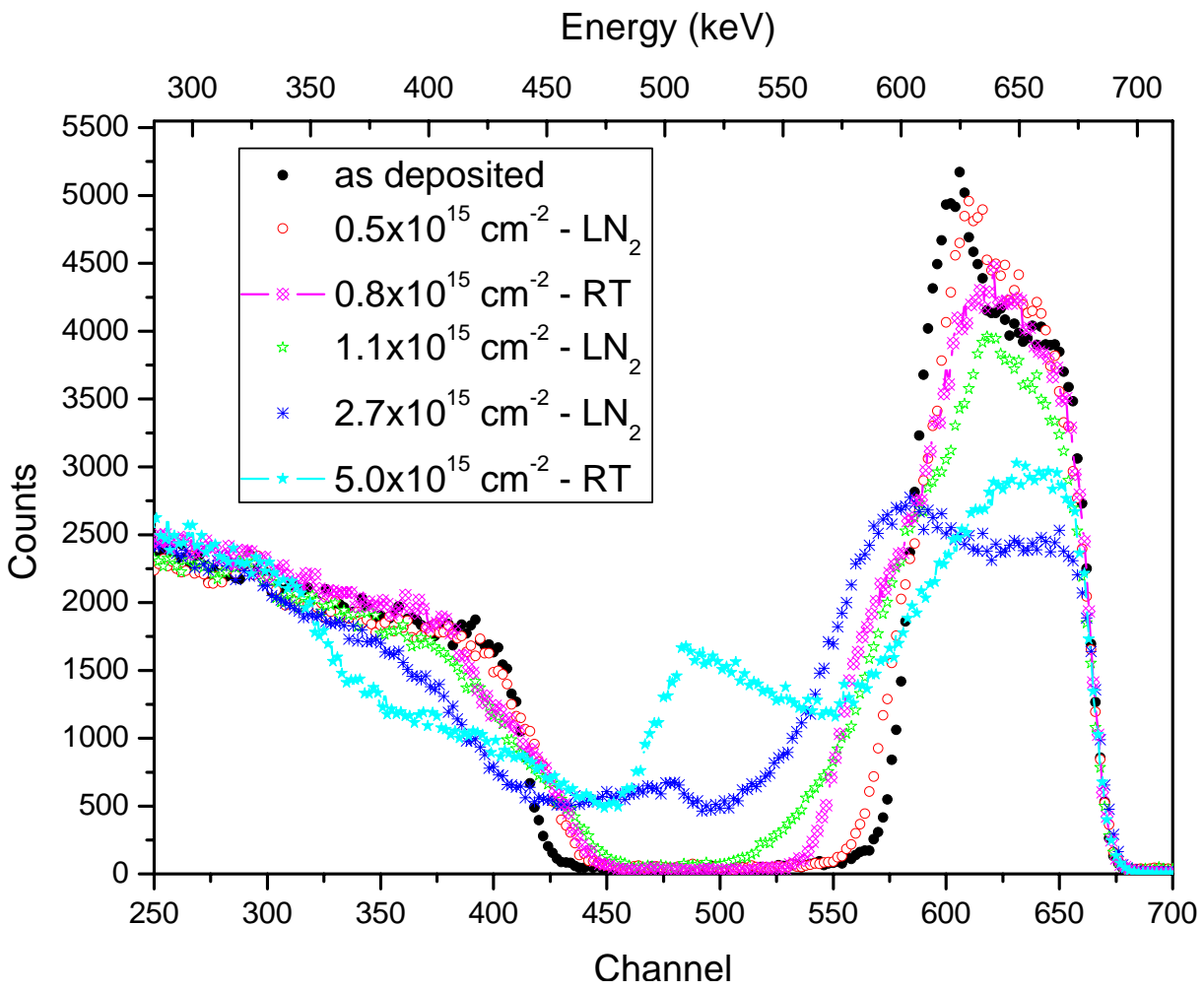

Fig 6.2.2 Selected raw RBS spectra measured before and after irradiation of the ${ }^{n a t} \mathrm{Fe}(45 \mathrm{~nm}) /{ }^{57} \mathrm{Fe}(20 \mathrm{~nm}) / \mathrm{Si}$ trilayers. The irradiations were performed at different fluences and two temperatures: liquid nitrogen $\left(\mathrm{LN}_{2}\right)$ and room temperature (RT), as indicated in the picture. 
Fig 6.2.2, which represents selected RBS spectra of the trilayer samples, shows that silicon reaches the sample surface when it is irradiated at the highest fluences $\left(2.7 \times 10^{15}\right.$ and $\left.5.0 \times 10^{15} \mathrm{~cm}^{-2}\right)$, which is indicated by a clear peak at channel 480 . For all other fluences the silicon and iron signals were separated. From the measured broadening of the silicon signal (Fig. 6.2.3a) the experimental mixing rate of $\Delta \sigma^{2} / \Phi=$

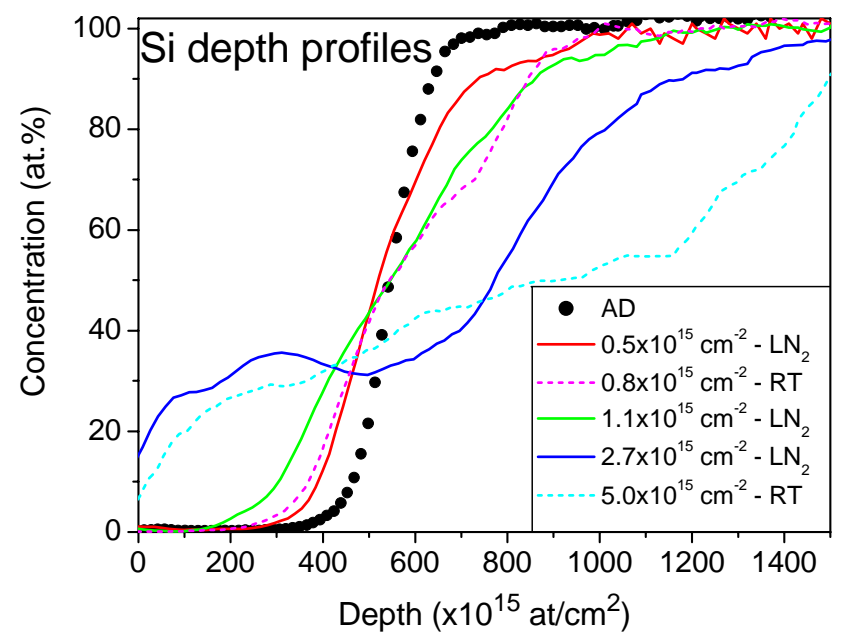

(a)

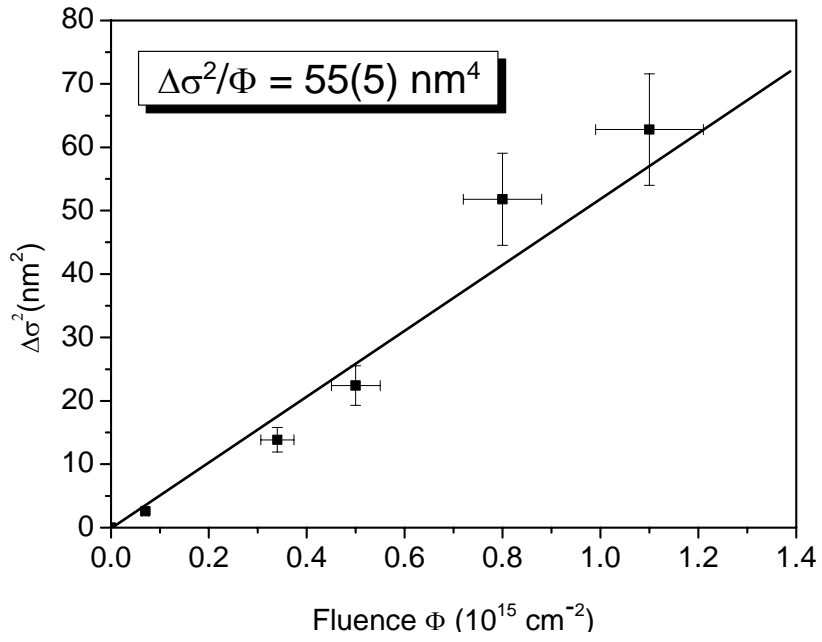

(b)

Figure 6.2.3 Broadening of the silicon depth profile (a) and interface variance of the mixed Fe/Si interface $\Delta \sigma^{2}$ as a function of the Xe ion fluence $\Phi(\mathrm{b})$. Also the slope of the linear curve is indicated $\left(\Delta \sigma^{2} / \Phi\right)$, labeled as mixing rate.

55(5) $\mathrm{nm}^{4}$ was calculated (Fig. 6.2.3b).

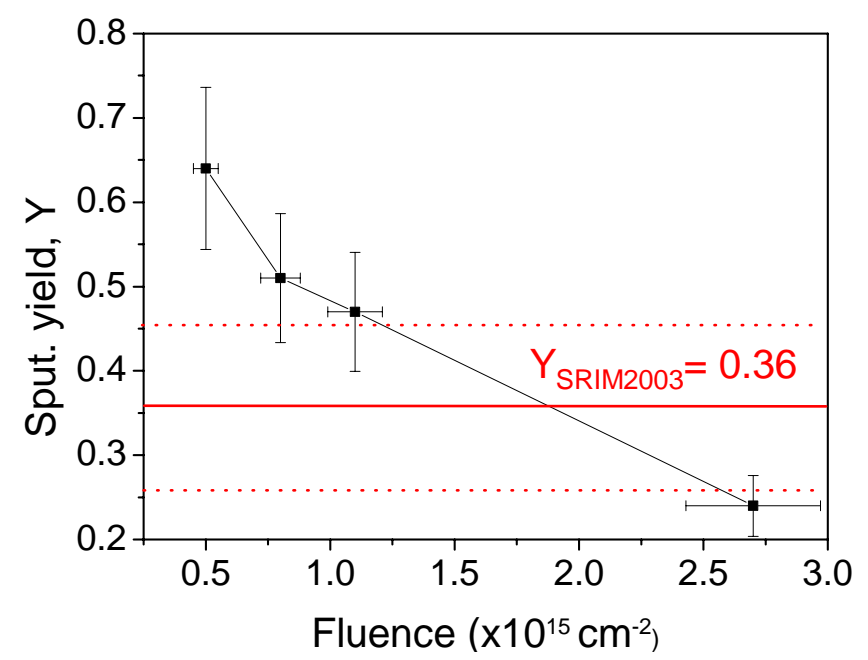

Figure 6.2.4 Calculated sputtering yield for the trilayers irradiated at different fluences, as well as SRIM result.
By integrating the iron depth profiles the sputtering yield, which is close to the SRIM predictions, was deduced. It decreases with increasing ion fluence (Fig 6.2.4), indicating the structural changes in the upper part of the sample, probably ironoxide formation [6-4] and iron-silicide. 
The deduced mixing rate of 55(5) $\mathrm{nm}^{4}$ is by one order of magnitude higher than the mixing rates measured for the $250-700 \mathrm{keV} \mathrm{Xe}^{+}$or $\mathrm{Au}^{+}$ions [6-5]. In these experiments, where nuclear stopping plays a major role, the mixing rate of the $\mathrm{Fe} / \mathrm{Si}$ bilayers was in the range of 5-9 $\mathrm{nm}^{4}$. The rather big mixing effect of the bilayers when electronic stopping is dominant was explained by a transfer of the energy deposited by the ions in the electronic subsystem to the lattice $[6-6,7,8]$. It was assumed that the energy deposited initially in the electronic subsystem within $10^{-15}-10^{-14}$ s gets transferred to the atomic subsystem by electron-phonon coupling in $10^{-13}-10^{-12} \mathrm{~s}$. As a result of this transfer, a rapid rise of the lattice temperature up to $10^{4} \mathrm{~K}$ occurs along the ion path (thermal spike). If a certain threshold of energy loss is exceeded, the material within few nanometers from the ion path melts for a duration of $10^{-12}-10^{-11}$ $\mathrm{s}$. The molten region rapidly quenches at a rate of about $10^{-14} \mathrm{~K} / \mathrm{s}$, forming latent tracks. It is interesting to note that in order to reach the melting point of $\mathrm{Si}$, the calculated critical value of the energy deposited due to electronic stopping is about 10 $\mathrm{keV} / \mathrm{nm}[6-9,10]$. Experimental proof of this result does not exist yet. The fact that no latent tracks in bulk Si were observed can be attributed to the recrystallization of the molten region.

The electronic energy loss in our experiment is sufficient to produce a molten region in both layers (Fe and Si). In this way, the conditions for forming thermal spikes are fulfilled, and interdiffusion of Fe and Si occurs in the molten region. The large mixing rate of 55(5) $\mathrm{nm}^{4}$ gave us evidence of this process.

It was reported that a significant diffusion of oxygen occurs during high-energy ion irradiations of iron [6-4]. Bearing this in mind, the decreasing sputtering yield for increasing ion fluence can be explained on the basis of these facts. The formation of a thin top layer of oxide reduces the sputtering yield by a factor of three.

In the RBS measurements no significant difference was observed in the samples irradiated at liquid nitrogen and room temperature.

Further information about irradiated bi/tri-layers was obtained by XRD and CEMS measurements.

The XRD measurements were performed in glancing incidence geometry, with an Xray source fixed at $3^{\circ}$. The measurements ranged from $17^{\circ}$ to $102^{\circ}$, with a step size of $0.1^{\circ}$ (see Fig 6.2.5). 


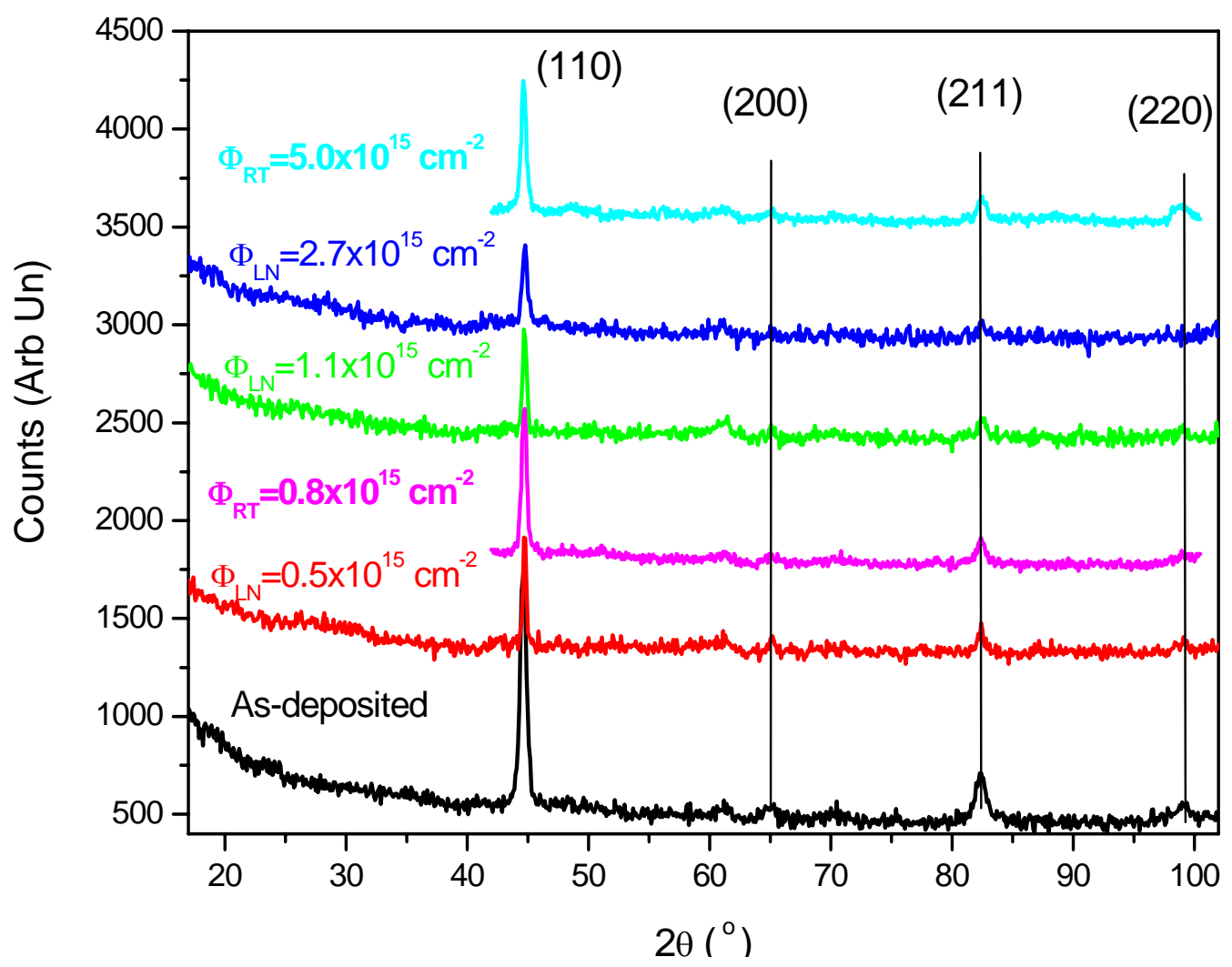

Figure 6.2.5 Glancing incidence XRD measurements of the as-deposited and irradiated samples; different reflections of the iron are indicated.

The XRD scans do not show any crystalline iron silicide phase. In all the samples the peak corresponding to the strongest reflection of iron (110) is present, as well as the (211) reflection. The intensities of both peaks decreased with increasing fluence, indicating a reduction in the crystalline iron phase and the formation of amorphous iron silicide phases.

Fig 6.2.6 shows the CEM spectra of the as-deposited and irradiated samples. Up to a fluence of $1.1 \times 10^{15} \mathrm{~cm}^{-2}$ a sextet with a hyperfine field of $33 \mathrm{~T}$ can be identified, that corresponds to $\alpha$-Fe. The intensity of this sextet in the CEM spectra decreased with the ion fluence, and finally disappeared in the spectrum taken of the sample irradiated at $2.7 \times 10^{15} \mathrm{~cm}^{-2}$. There is another sextet with a smaller hyperfine field of 27.7(3) $\mathrm{T}$, which is broad. This indicates that an amorphous ferromagnetic iron-silicon phase has formed. This broad sextet appears after irradiation at the lowest fluence, but disappears after irradiation at the highest fluence, where only two broad doublets with 
central shifts of $0.19(4)$ and $0.21(3) \mathrm{mm} / \mathrm{s}$ and quadrupole splittings of $0.89(6)$ and $0.53(7) \mathrm{mm} / \mathrm{s}$, respectively, were observed.

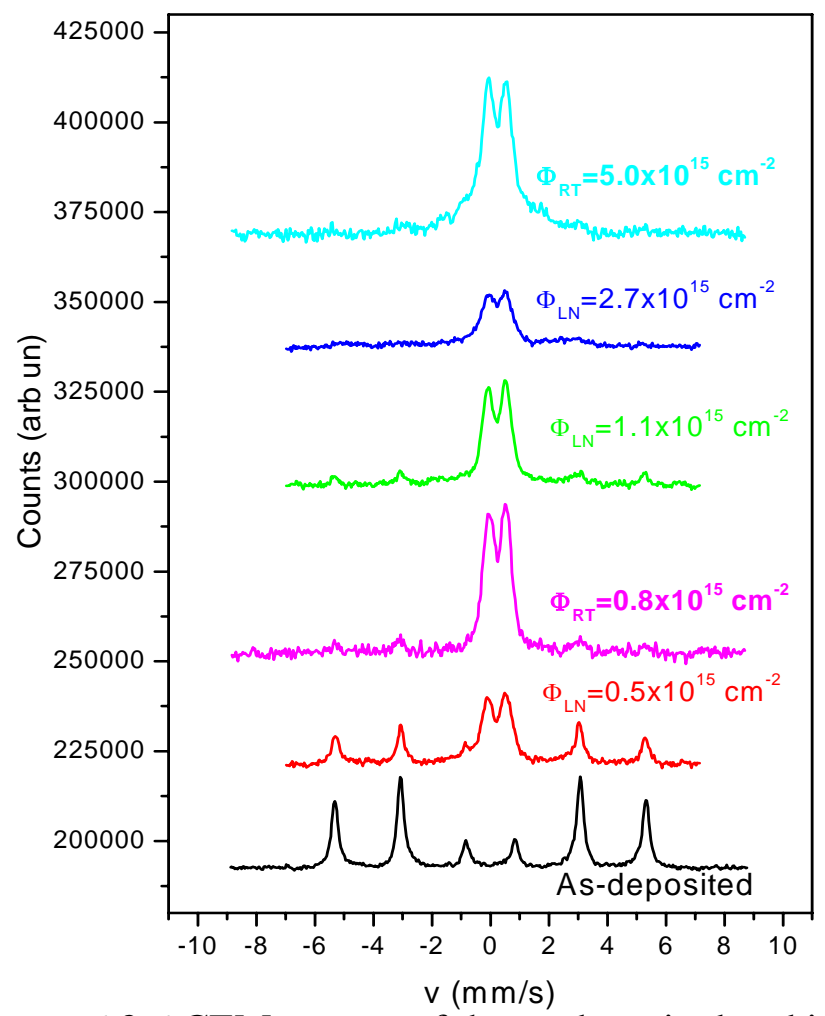

Figure 6.2.6 CEM spectra of the as-deposited and irradiated samples.

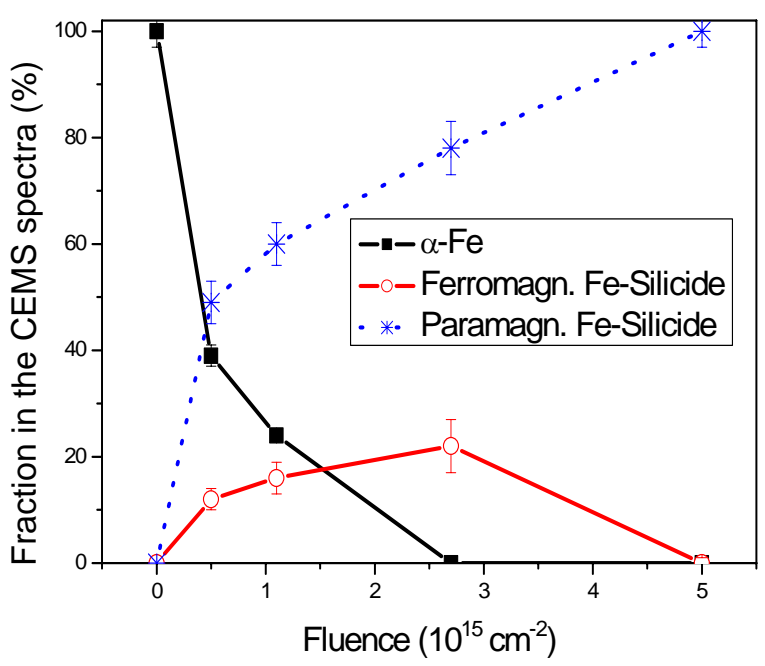

Figure 6.2.7 Evolution of the relative fraction of the amorphous ferromagnetic and paramagnetic phase and the crystalline $\alpha$-Fe phase for different irradiation fluences. 
Figure 6.2.7 presents the evaluation of the ferromagnetic and paramagnetic contributions to the spectra.

The transformation from the ferromagnetic crystalline $\alpha$-Fe and amorphous Fesilicide phase to the paramagnetic Fe-silicide phases indicates an increasing presence of silicon atoms in the vicinity of ${ }^{57} \mathrm{Fe}$. The counting statistics was not sufficient to dissolve particular paramagnetic phases.

Comparing the CEMS results with those obtained by XRD measurements it can be concluded that crystalline iron remains on the surface (i.e. on the layer of natural iron), while the region close to the iron/silicon interface is completely mixed and amorphous, in the interface region there is ${ }^{57} \mathrm{Fe}$, and the CEMS results come from this region, while the XRD experiments provide data of the whole sample.

Assmann et al. performed a similar CEMS experiment, in which ${ }^{\text {nat }} \mathrm{Fe}(30 \mathrm{~nm}) /{ }^{57} \mathrm{Fe}(10$ $\mathrm{nm}$ )/Si trilayers were irradiated with $243 \mathrm{MeV} \mathrm{Au,} 210 \mathrm{MeV}$ I and $160 \mathrm{MeV}$ Ag ions [6-10]. Two paramagnetic doublets with hyperfine parameters similar to ours were identified and associated with the metastable, high-temperature crystalline $\alpha-\mathrm{FeSi}_{2}$ phase. Also, this group ruled out any solute iron atoms in Si. As can be seen from our RBS results, significant mixing occurs during irradiation with $350 \mathrm{MeV}$ Au ions. The XRD measurements gave no indication of any crystalline phase, and finally CEMS showed the presence of two paramagnetic doublets with a large broadening, indicating the amorphous nature of those iron-silicide(s). The above discrepancy may possibly be attributed to the lower ion energies and fluences $\left(\leq 5.4 \times 10^{14} \mathrm{~cm}^{-2}\right)$ used by Assmann et al.

Similar results were achieved by Bauer, Dufour et al. [6-11], using ${ }^{57} \mathrm{Fe}(4.5 \mathrm{~nm}) / \mathrm{Si}$ (3.5 nm) multilayers, which were irradiated with $\mathrm{U}(650 \mathrm{MeV})$, Xe $(858 \mathrm{MeV})$ or $\mathrm{Kr}$ (736 MeV) ions up to a fluence of $10^{14} \mathrm{~cm}^{-2}$. An important difference between their and our CEMS results is the relative contribution of the amorphous ferromagnetic phase. At the highest fluence we used $\left(5 \times 10^{15} 1 / \mathrm{cm}^{2}\right)$ this phase had vanished, while Bauer, Dufour et al. recorded a constant increase in the fraction of this phase with increasing fluence (up to $1 \times 10^{14} \mathrm{~cm}^{-2}$ ).

For those samples irradiated at a fluence of $0.5 \times 10^{15} \mathrm{~cm}^{-2}$ a relative contribution to the CEM spectra was sufficient to record MOMS spectra (see Fig 6.2.8). 


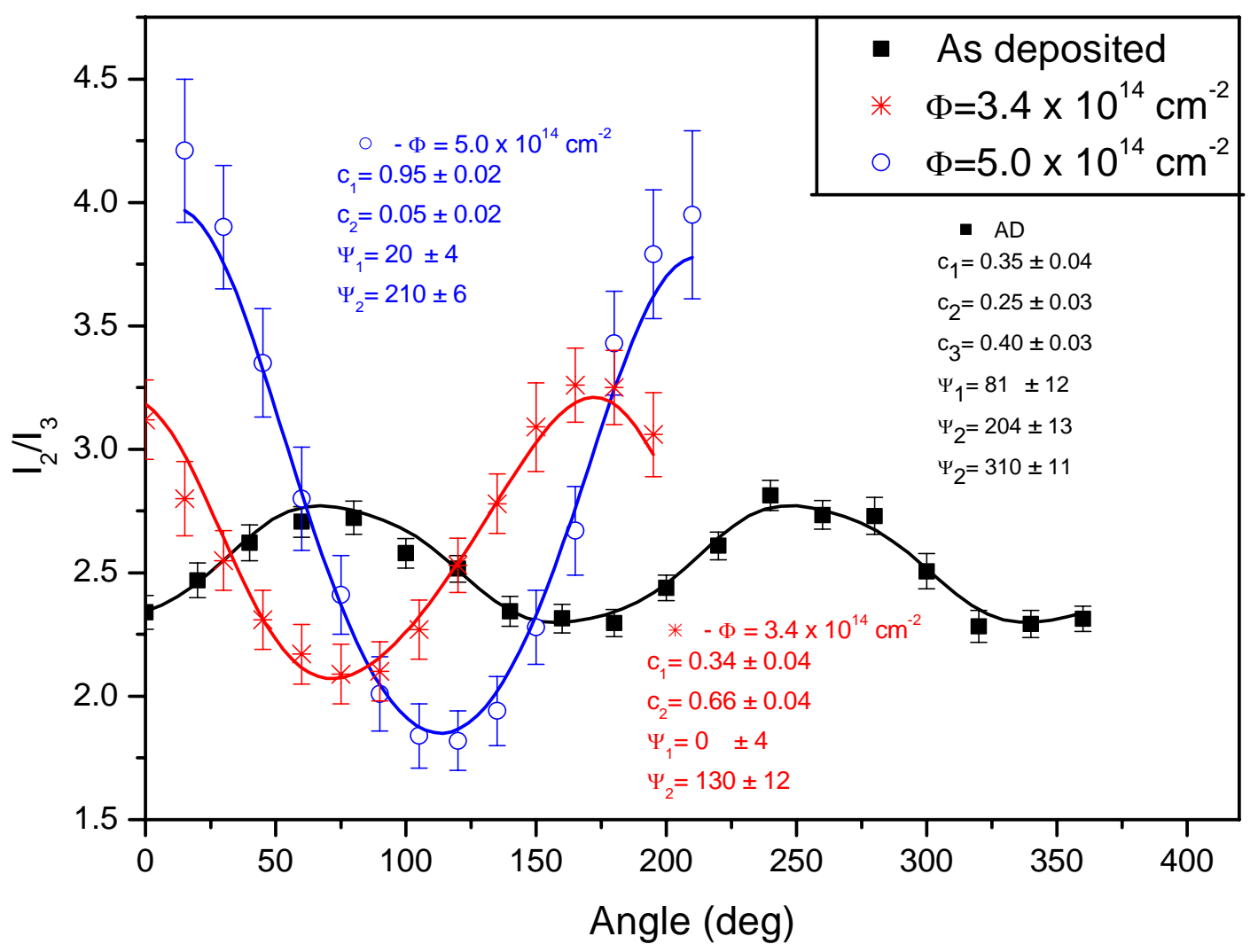

Figure 6.2.8 MOMS spectra, and corresponding fits, of the as-deposited sample and of the sample irradiated with 3.4 and $0.5 \times 10^{15} \mathrm{Au}$-ions $/ \mathrm{cm}^{2}$.

The relative ratio of the intensities of the second and third peak was fitted with the following function [6-13]:

$$
\frac{I_{2}}{I_{3}}=4 \sum_{i=1}^{n} c_{i} \frac{1-\sin ^{2} \alpha \sin ^{2}\left(\varphi-\Psi_{i}\right)}{1+\sin ^{2} \alpha \sin ^{2}\left(\varphi-\Psi_{i}\right)} ; \sum_{i=1}^{n} c_{i}=1,
$$

Small variations of the $I_{2} / I_{3}$ values in the as-deposited sample indicate the isotropic inplane spin distribution. In order to fit this spectrum, three terms from Eq. 6.2.1 were used. Almost the same contributions $C_{i}$ were found and the orientations were separated by approximately $120^{\circ}$. This is a clear sign of near-isotropic distributions of the inplane spins. With the irradiated sample, the differences in the $\mathrm{I}_{2} / \mathrm{I}_{3}$ ratios are much more pronounced, and a clear preferential orientation of the spin is present. The biggest contribution to the CEMS/MOMS spectra comes from the conversion electrons originating from the ${ }^{57} \mathrm{Fe} / \mathrm{Si}$ interface region. In order to check the magnetic properties of the near-surface region, MOKE measurements were performed. Figure 
6.2.8 shows the results of the MOKE measurements of the samples irradiated up to $1.1 \times 10^{15} \mathrm{~cm}^{-2}$.
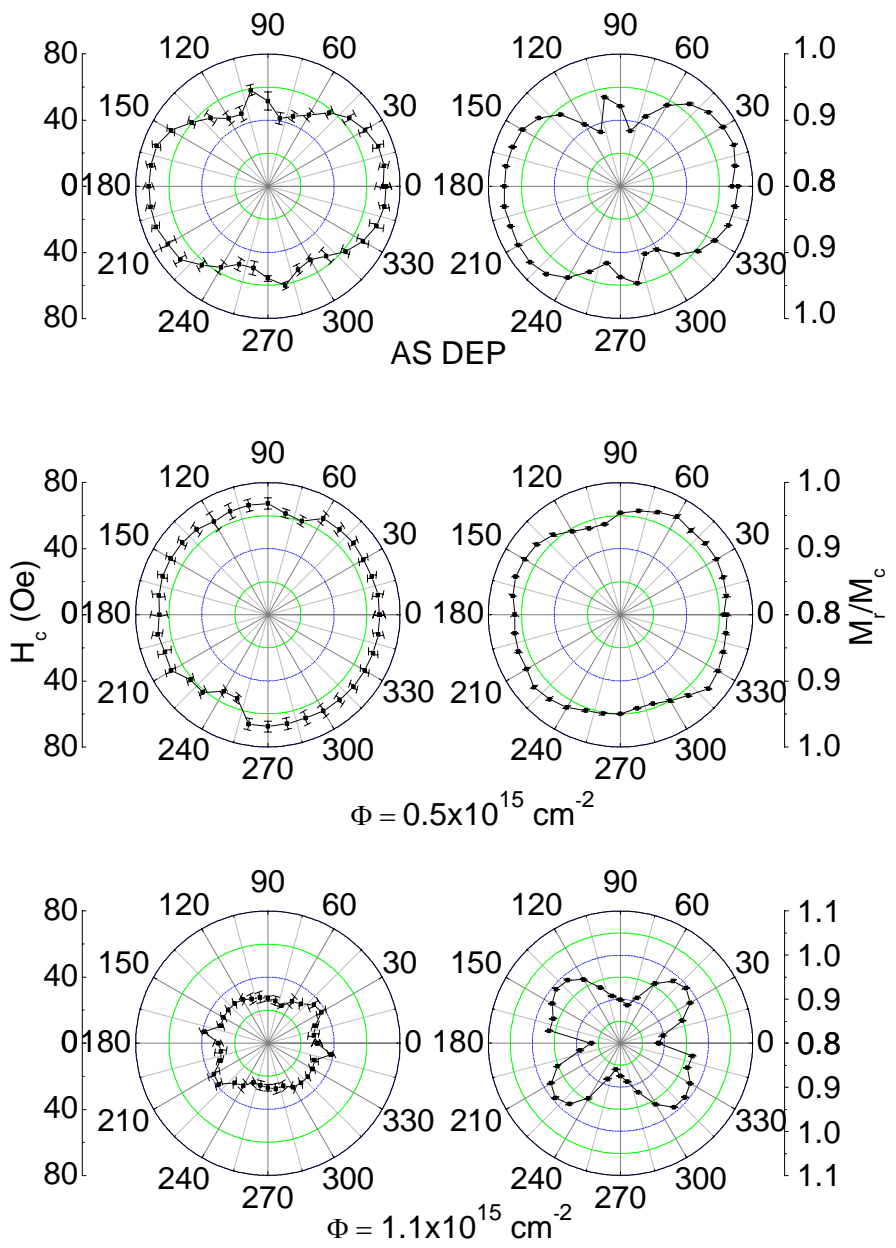

Figure 6.2.8 Fluence dependence of the coercivity field $\mathrm{H}_{\mathrm{c}}$ and relative magnetic remanence $\mathrm{M}_{\mathrm{r}} / \mathrm{M}_{\mathrm{s}}$ obtained by MOKE measurements.

The as-deposited Fe layer shows uniaxial magnetic anisotropy, coupled with a slight fourfold anisotropy. The component with four-fold symmetry grew to $5 \times 10^{14}$ and $1.1 \times 10^{15} \mathrm{~cm}^{-2}$, but finally isotropic magnetization was found for the highest Au-ion fluences of 2.7 and $5 \times 10^{15} \mathrm{~cm}^{-2}$. These findings differ from the ones achieved by the MOMS technique, which indicates the difference in the structural changes in the surface and the interface region of the samples, which is due to the irradiation with swift heavy ions. 


\section{References}

\section{Chapter 1: Summary}

[1-1] V. Milinovic, N. Bibibc, S. Dhar, M. Siljegovic, P. Schaaf, K. P. Lieb, Appl. Phys. A, 79 2093-2097 (2004).

[1-2] N. Bibic, V. Milinovic, S. Dhar, K. P. Lieb, M. Milosavljevic, M. Siljegovic, D. Perusko, P. Schaaf, Thin Solid Films, 459 (2004) 23-27.

[1-3] K. P. Lieb, K. Zhang, V. Milinovic, P. K. Sahoo and S. Klaumünzer, Nucl. Instr. Meth. $B$ - in press

[1-4] V. Milinovic, K. P. Lieb, P. K. Sahoo, P. Schaaf, K. Zhang, S. Klaumünzer and M. Weisheit, Appl. Surf. Sci. - in press

Chapter 2: Introduction

[2-1] W. D. Cussins, Proc. Phys. Soc., 368 (1955) 213.

[2-2] G. Dearneley, Nucl. Instr. Meth. B, 50 (1990) 358.

[2-3] J. Lindhard, M. Scharff, Mat. Fis. Medd. Dan. Vid. Selsk., 33 No. 14(1963).

[2-4] P. D. Townsend, J. C. Kelly and N. E. W. Hartley, Ion Implantation, Sputtering and Their Applications, Academic Press Inc., London (1976).

[2-5] J. F. Ziegler, J. P. Biersack, U. Littmark, The Stopping and Range of Ions in Solids, Pergamon Press, New York, 1985.

http://www.srim.org

[2-6] Y. T. Cheng, Mat. Sci. Rep., 5 (1990) 45.

[2-7] P. Sigmund, Phys. Rev., 184 (1969) 383.

[2-8] P. Sigmund and A. Gras-Marti, Nucl. Instr. Meth., 182/183 (1981) 25.

[2-9] W. L. Johnson, Y. T. Cheng, M. van Rossum and M. A. Nicolet, Nucl. Instr. Meth., B 7/8 (1985) 657.

[2-10] P. Borgersen, D. A. Lilenfeld and H. H. Johnson, Appl. Phys. Lett., 57 (1990) 1407

[2-11] G. Rixecker, P. Schaaf, and U. Gonser, Phys. Stat. Sol. (a) 139 (1993) 309, and references there-in.

[2-12] P. Mangin and G. Marchal, J. Appl. Phys., 49 (1978) 1709.

[2-13] J. Desimoni and F. H. Sanchez, Hyp. Int., 122 (1999) 277; 113 (1998) 403.

[2-14] M. Milosavljevic, S. Dhar, P. Schaaf, N. Bibic, M. Han, K. P. Lieb, Appl. Phys. A 71 (2000) 43.

[2-15] M. Milosavljevic, S. Dhar, P. Schaaf, N. Bibic, M. Han, K. P. Lieb, Nucl. Instr. Meth., B 178 (2001) 4474.

[2-16] P. Schaaf, M. Milosavljevic, S. Dhar, N. Bibic, K. P. Lieb, M. Wölz and G. Principi, Hyp. Int., 139/140 (2002) 615.

[2-17] S. Dhar, P. Schaaf, N. Bibic, E. Hooker, M. Milosavljevic, K. P. Lieb, Appl. Phys. A 76 (2003) 773.

[2-18] S. Dhar, P. Schaaf, K. P. Lieb, N. Bibic, M. Milosavljevic, T. Sajavaara, J. Keinonen, A. Traverse, Nucl. Instr. Meth., B 205 (2003) 741.

[2-19] S. Dhar, M. Milosavljevic, P. Schaaf, N. Bibic, K. P. Lieb, "Ion Beam Mixing and silicide formation in Fe/Si and Ta/Si bilayers ", Proceedings 21st International Conference and Summer School on the Physics of Ionized Gases 
(SPIG2002), August 25-30, 2002, Sokobanja, Belgrade, Yugoslavia, “The Physics of Ionized Gases",

Eds.: N. Bibic, T. Grozdanov, and M. Radovic (Vinca Institute of Nuclear Science, Belgrade, 2003) 189-206.

[2-20] M. Milosavljevic, S. Dhar, P. Schaaf, N. Bibic, Y. L. Huang, M. Seibt, and K. P. Lieb, J. Appl. Phys., 90 (2001) 4474.

Chapter 3: Experimental techniques

[3-1] J. F. Harbsmeier, Dissertation, Göttingen 2000.

[3-2] A. Dobrosavljevic, M. Milosavljevic, N. Bibic, A. Efremov, Rev. Sci. Instrum. 71 (2000) 786.

[3-3] H. U. Krebs, Int. J. Non-Equil. Proc., 10 (1997) 3.

[3-4] J. F. Ziegler and W. K. Chu, Atomic Data and Nuclear Data Tables, 13 (1974) 463.

[3-5] F. Hubert, A. Fluery, R. Bimbot and H. Gauvin, Atomic Data and Nuclear Data Tables, 46 (1990) 1.

[3-6] W. K. Chu, J. W. Mayer, M. A. Nicolet, Backscattering Spectrometry, Academic Press, New York, 1978.

[3-7] J. R. Tesmer, M. Nastasi, Handbook of Modern Ion Beam Materials Analysis, Material Science Society, Pittsburgh, 1995.

[3-8] K. P. Lieb, Contemp. Phys., 40 (1999) 385.

[3-9] R. L. Doolittle, Nucl. Instr. Meth. B, 15 (1986) 227. http://www.genplot.com/

[3-10] N. P. Barradas, C. Jeynes, R. P. Webb, Appl. Phys. Lett. 71 (1997) 291. http://www.ee.surrey.ac.uk/SCRIBA/ibc/ndf/

[3-11] http://www.srim.org/\#IBA

[3-12] M. Uhrmacher, K. Pampus, F. J. Bergmeister, D. Purschke, K. P. Lieb, Nucl. Instr. Meth. B, 9 (1985) 234.

[3-13] P. Schaaf, A. Krämer, L. Blaes, G. Wagner, F. Aubertin, and U. Gonser, Nucl. Instr. and Meth. B 53 (1991) 184.

[3-14] R. L. Mössbauer, Z. Physik, 151 (1958) 124.

[3-15] J. J. Spijkermann, Conversion Electron Mössbauer Spectroscopy in Mössbauer Effect Methodology, Vol. 7, I. J. Gruvermann ed., Plenum Press, New York London, (1981).

[3-16] U. Gonser, Mössbauer Spectroscopy in Microscopic Methods in Metals, Springer Verlag (1986).

[3-17] http://www.isapps.ca/recoil/

[3-18] P. Schaaf, C. Illgner, M. Niederdrenk and K. P. Lieb, Hyp. Int. 95 (1995) 199225.

[3-19] P. Schaaf, Prog. in Mat. Sc., 47 (2002) 1.

[3-20] M. Kopcewicz, J. Jagilski, A. Turos, D. L. Williamson, J. Appl. Phys., 71 (1992) 4217.

[3-21] E. U. Concon and G. H. Shortley, The Theory of Atomic Spectra, Cambridge Univ. Press, 1935.

[3-22] P. Schaaf, G. A. Müller, E. Carpene and M. Kahle, Hyp. Int., 144/145 (2002) 129.

[3-23] G. A. Müller, R. Gupta, K. P. Lieb and P. Schaaf, Appl. Phys. Lett., 82 (2003) 73.

[3-24] G. A. Müller, doctoral thesis, Göttingen 2004. 
http://webdoc.sub.gwdg.de/diss/2004/mueller_georg/

[3-25] M. Faraday, On the magnetization of light and illumination of magneticlines of force, Phil. Trans. Royal. Soc. (london) 136 (1846) 1.

[3-26] J. Kerr: On rotation of the plane of polarization by reflection from the polar of a magnet, Phil. Mag. (5) 3 (1877) 321.

[3-27] P. N. Argyres, Phys. Rev., 97 (1955) 334.

[3-28] B. R. Cooper, Phys. Rev., 139 (1965) A1504.

[3-29] A. Hubert and R. Schäfer, Magnetic domains - the analysis of magnetic microstructures, Springer - Verlag Berlin Heidelberg, 1998.

[3-30] R. Jenkins and R. L. Snyder, Introduction to X-ray powder diffractometry, John Wiley \& Sons, Inc., 1996.

[3-31] CD-ROM: PDF-2 Data Base (Sets 1-47), PDF-2 47 6A JUN97; JCPDS International Center for Diffraction Data.

Chapter 4: Nitrogen irradiation and thermal annealing

[4-1] L. Rissanen, S. Dhar and K. P. Lieb, Phys. Rev. B 63 (2001) 155411.

[4-2] R. W. Cahn, P. Haasen, Physical metallurgy, Amsterdam/Oxford/New York/Tokio: North-Holland Physics Publishing, 1983.

[4-3] C. R. Brooks, Principles of the surface treatment of steels, Lancaster, Basel; Technomic Publishing Company, 1992.

[4-4] J. Kunze, Nitrogen and carbon in iron and steel, Berlin; Akademie Verlag, 1990.

[4-5] H. A. Wriedt, N. A. Gokcen, R. H. Nafziger, T. B. Massalski, Binary alloy phase diagrams; American Society for Metals (ASM), Metals Park, OH.

[4-6] P. Schaaf, C. Illgner, M. Niederdrenk and K. P. Lieb, Hyp. Int. 95 (1995) 199225; and references there in.

[4-7] H. U. Krebs, Int. J. Non-Equil. Proc., 10 (1997) 3.

[4-8] A. Dobrosavljevic, M. Milosavljevic, N. Bibic, A. Efremov, Rev. Sci. Instrum. 71 (2000) 786.

[4-9] M. Kopcewicz, J. Jagielski, A. Turos, D. L. Williamson, J. Appl. Phys. 91 (2002) 6465.

[4-10] N. Bibic, V. Milinovic, S. Dhar, K. P. Lieb, M. Milosavljevic, M. Siljegovic, D. Perusko, P. Schaaf, Thin Solid Films, 459 (2004) 23-27.

[4-11] Y. T. Cheng, Mat. Sci. Rep. 5 (1990) 45.

[4-12] W. Bolse, Mat. Sci. Eng. R 12, 40 (1994); Nucl. Instr. Meth. B, 148 (1999) 83.

[4-13] T. Corts, W. Bolse, A. Traverse, Nucl. Instr. Meth. B, 80 (1993) 167.

[4-14] S. Dhar, L. Rissanen, K. Engel, M. Wenderoth, K. P. Lieb, Nucl. Instr. Meth. $B, 178$ (2001) 297.

[4-15] V. Milinovic, N. Bibic, S. Dhar, M. Siljegovic, P. Schaaf, K. P. Lieb, Appl. Phys. A, 79 (2004) 2093-2097.

[4-16] M. Niederdrenk, P. Schaaf, K. P. Lieb, O. Schulte, J. Alloys Compd. 237 (1996) 81.

[4-17] M. J. Clauser, Solid State Commun. 8 (1970) 751.

[4-18] J. Desimoni, F. H. Sanchez, Hyp. Int. 122 (1999) 277. 
Chapter 5: Effects of substrate amorphization and ion charge

[5-1] J. F. Ziegler, J. P. Biersack, U. Littmark, The Stopping and Range of Ions in Solids, Pergamon Press, New York, 1985. http://www.srim.org

[5-2] A. Dobrosavljevic, M. Milosavljevic, N. Bibic, A. Efremov, Rev. Sci. Instrum. 71 (2000) 786.

[5-3] K. P. Lieb, Contemp. Phys., 40 (1999) 385.

[5-4] N. P. Barradas, C. Jeynes, R. P. Webb, Appl. Phys. Lett. 71 (1997) 291. http://www.ee.surrey.ac.uk/SCRIBA/ibc/ndf/

[5-5] S. Dhar, P. Schaaf, K. P. Lieb, N. Bibic, M. Milosavljevic, T. Sajavaara, J. Keinonen, A. Traverse, Nucl. Instr. Meth., B 205 (2003) 741.

[5-6] N. Stolterfoht, J. H. Bremer, R. D. Muino, Int. J. Mass. Spectrom., 192 (1999) 425.

Chapter 6: Swift ion irradiation with $350 \mathrm{MeV}$ Au ions

[6-1] J. F. Harbsmeier, Dissertation, Göttingen 2000.

[6-2] http://www.hmi.de

[6-3] J. F. Ziegler, J. P. Biersack, U. Littmark, The Stopping and Range of Ions in Solids, Pergamon Press, New York, 1985. http://www.srim.org

[6-4] D. K. Avasthi, W. Assmann, A. Tripathi, S. K. Srivastava, S. Ghosh, F. Grüner and M. Toulemonde, Phys. Rev. B 68 (2003) 153106.

[6-5] S. Dhar, P. Schaaf, N. Bibic, E. Hooker, M. Milosavljevic, K. P. Lieb, Appl. Phys. A, 76 773-780 (2003).

[6-6] K. Izui, J. Phys. Soc. Jpn. 20945 (1965).

[6-7] K. Markele, Phys. Rev. Lett. 9150 (1962).

[6-8] Z. G. Wang, C. Dufour, E. Paumier, M. Toulemonde, J. Phys.: Condens Matt. 6 (1994) 6773.

[6-9] S. Kraft, B. Schattat, W. Bolse, S. Klaumünzer, F. Harbsmeier, A. Kulinska, A. Löffl, J. Appl. Phys. 91 (2002) 1129.

[6-10] M. Toulemonde, C. Dufour, E. Paumier, Phys Rev B 46 (1992) 14362.

[6-11] W. Assmann, M. Dobler, D. K. Avasthi, S. Kruijer, H. D. Mieskes, H. Nolte, Nucl. Instr. Meth. B, 146 (1998) 271.

[6-12] P. Bauer, C. Dufour, C. Jaouen, G. Marchal, J. Pacaud, J. Grilhe, J. C. Jousset, J. Appl. Phys. 81 (1997) 116.

[6-13] G. A. Müller, R. Gupta, K. P. Lieb and P. Schaaf, Appl. Phys. Lett., 82 (2003) 73. 


\section{Acknowledgements}

In the first place I would like to thank Prof. Dr. Dr. h.c. Klaus Peter Lieb for giving me the opportunity to work on this thesis, constant help and readiness to discuss any type of scientific problem. Without support of Prof. Lieb most of this work would not exist. The time that I spend on regular meetings with Prof. Lieb is the most valuable time during my stay in Göttingen.

I am very grateful to Prof. Dr. Angela Ritzy, as well as Prof. Dr, H. Krebs, PD Dr. Carsten Ronning, Prof Dr. Stefan Mayr and Prof. Dr. Hans Hofsäß for their interest in this work.

I also want to thank PD Dr. Peter Schaaf for his introducing me to the fascinating world of Mössbauer spectroscopy.

I also express my deepest gratitude to Dr. Nataša Bibić and Dr. Momir Milosavljević for fruitful collaboration and permanent support.

I want to express my thanks to Dr. Kun Zhang for his friendly help and his pleasant collaboration concerning MOKE experiments.

I address my thanks to the former members of the group, Dr. Alexander Müller, Dr. Stanislawa Gasiorek, Dr. Sankar Dhar and Dr. Pratap Sahoo for the nice atmosphere they created inside the group.

Many thanks to Detlef Purschke and Holger Schebela for dismounting/mounting IONAS. Once more, many thanks to Detlef for running IONAS (no protons in the beam during RBS measurements; never!).

Thanks are addressed to the members of the electronic and precision engineering workshop, especially to their heads Kurt Schemmeling and Klaus Arndt.

My sincere thanks to all members of the II. Phys. Inst. who always make the working atmosphere enjoyable (special thanks to Helge Kröeger for the “mensa time”, and my roommate Christian Lange: for almost one year he is able to stand my strange jokes). Frau Christa Wohlfarth and Lucie Hamdi are kindly acknowledged for their continuous help with all the small and big administrative problems. I am obliged to Lucie for valuable corrections of the English language (not only word order and "the", “a”, “an” - fileN dank Lucie). 
Of course that I did not forget Dr. Michael Uhrmaher. The list that I am greatfull to him will be long, so I will reduce it to: support, advices, small talks (physic/non physic topics), constant good mood.....

Last but not least, I would like to express the gratitude towards my parents Radosav and Slobodanka Milinović for their encouragement and understanding. 


\section{Curriculum Studiorum}

Personal Data:

- name: Velimir Milinović

- birthplace: Belgrade - Serbia and Montenegro

- birthdate: 15. 04. 1970

- citizenship: Serbian

Education:

- 1977-1985: Elementary School (Serbia and Montenegro)

- 1985-1989: High School (Serbia and Montenegro)

- 1989-1990: Military Service

- 1990-1999: Physics studies at the University of Belgrade (Serbia and Montenegro)

- 1999-2002: Master's studies at the University of Belgrade (Serbia and Montenegro)

- 2002-2005: Physics PhD student at the II. Physikalisches Institut, University of Göttingen (Germany)

Teaching activities

- 2005: Fortgeschrittenenpraktikum at the II. Physikalisches Institut, University of Göttingen (Germany) 